\title{
Model-Order Reduction of Massively Coupled Parameterized Systems via Clustering
}

\author{
by \\ Jaydip Kathrotiya \\ A thesis submitted to the Faculty of Graduate and Postdoctoral Affairs \\ in partial fulfillment of the requirements for the degree of \\ Master of Applied Science \\ in \\ Electrical and Computer Engineering
}

Carleton University

Ottawa, Ontario, Canada

(C) 2021

Jaydip Kathrotiya 


\section{Abstract}

Parametrized Model Order Reduction (PMOR) methods reduce the full equations for large parametric networks into a reduced number of parametrized equations. The reduced model is obtained to match the variations in the response of the original full circuit due to the variations in its key design parameters. Applying the existing PMOR techniques to parametrized systems with many inputs often results in extremely large and dense reduced-order models.

This thesis presents a new approach to construct parametrized reduced-order models for multi-parameter networks with many input/output terminals. The new method leverages the efficient application of the multi-parameter moment-matching-based approach by exploiting the superposition paradigm. By clustering the full equations into subsystems, the proposed method constructs orthogonal bases to reduce each subsystem. The overall reduced parametrized macromodel is constructed by aggregating the resulting reduced parametrized subsystems. Solving the resulting overall reduced system to obtain the variations in the original circuit response yields significant computational savings.

The massively coupled interconnect structures as pertinent examples for multiport designs are used to describe the development of the algorithm. However, the application of the proposed method is not limited to the interconnect structures. It can be applied to the parametric model-order reduction of any general multi-parameter multiport linear system. 
The presented algorithm is an effective means to manage the Monte Carlo simulations of large multiport parametrized linear networks. It is also highly suited for multithreading implementation and thus facilitates parallel time-domain variability analysis. The validity and efficiency of the proposed algorithm are demonstrated through computational results. 


\section{Acknowledgments}

I would like to extend my sincere thanks and appreciation to my supervisor, Professor Michel Nakhla, who constantly pushed me to be professional and do the right thing despite the challenges I encountered. Without his persisting help, the task of completing this thesis could never have been accomplished.

I would also like to express my sincere gratitude and appreciation to my cosupervisor Dr. Behzad Nouri. Having encouraging words and thoughtful, detailed feedback from you has been very important to me. I am particularly grateful for the time you have taken out of your schedules to complete this research and make this project possible.

I am fortunate to have been a part of the CAD group. Thank you so much for your support through thick and thin. With such an organized, open, and helpful support system, every task, regardless of its difficulty, feels achievable.

Finally, I want to extend my sincere gratitude to my family for their tremendous support and hope, which made this thesis possible for me. Without you, this thesis would not have been possible. Thank you for your support and hope. 


\section{Table of Contents}

Abstract $\quad$ ii

Acknowledgments $\quad$ iv

Table of Contents $\quad$ v

List of Tables viii

List of Figures $\quad$ ix

List of Acronyms $\quad$ x

List of Symbols $\quad$ xii

1 Introduction 1

1.1 Background and Motivation . . . . . . . . . . . . . 1

1.2 Objectives and Contributions . . . . . . . . . . . . . 2

1.3 Organization of the Thesis . . . . . . . . . . . . . 3

2 Formulation of Circuit Equations 5

2.1 MNA Matrix Equations . . . . . . . . . . . . . . . . 5

2.1.1 MNA Formulation of Linear Circuits . . . . . . . . . . . 6

2.2 Properties of MNA Matrices . . . . . . . . . . . . 8

2.3 Circuit transfer function . . . . . . . . . . . . . . . . . . . 8 
2.4 Stability of Linear Designs . . . . . . . . . . . . . . . . . . 11

3 Model Order Reduction Techniques 12

3.1 The Concept of MOR . . . . . . . . . . . . . . . . 13

3.2 MOR for Linear Circuits . . . . . . . . . . . . . . . . . . . . 13

3.3 Construction of Projection Matrix . . . . . . . . . . . . . . 16

3.3.1 Krylov-Subspace Based Methods . . . . . . . . . . . 16

3.3.2 Arnoldi Algorithm . . . . . . . . . . . . . . . 18

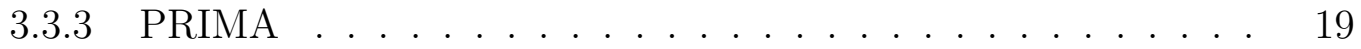

3.4 Summary and Discussions . . . . . . . . . . . . . . . . . . . 20

4 MOR for Parametric Circuits $\quad 21$

4.1 General Formulation of Parametric Circuits . . . . . . . . . . . . . . 22

4.1.1 Multi-parameter Moments of Parametric Circuits . . . . . . . 25

4.2 Reduction Scheme for Parametric Circuits . . . . . . . . . . . . . 28

4.3 Construction of Projection Matrix . . . . . . . . . . . . . . 28

4.3.1 Explicit Moment-Matching Based Approach . . . . . . . . . . 29

4.3.2 Implicit Moment-Matching Based Approach . . . . . . . . . . 31

4.4 Order Growth and Computational Complexity . . . . . . . . . . . 36

4.5 Summary and Discussions . . . . . . . . . . . . . . . . . . . . 37

5 Reduction of Massively Coupled Interconnect Structures: Cluster$\begin{array}{ll}\text { ing Scheme } & 38\end{array}$

5.1 MOR for Multi-Input Networks . . . . . . . . . . . . . . . . 39

5.2 MOR for MTL . . . . . . . . . . . . . . . . . 40

5.3 Reduction via Clustering . . . . . . . . . . . . . . . . 41

5.3.1 Formulation of the Reduced-order Models based on Clustering 43

5.4 Properties of Clustering Technique . . . . . . . . . . . 46 
5.4.1 Moments Preservation ............... . 46

5.4 .2 Stability .......................... 47

6 Parametric Model-Order Reduce of Multi-port Networks via Clus$\begin{array}{ll}\text { tering } & 49\end{array}$

6.1 Parametrized Circuit Formulation . . . . . . . . . . . . . . 50

6.2 Multi-parameter Moments of Circuit Response . . . . . . . . . . . . 51

6.3 Parametric Reduced-Order Model . . . . . . . . . . . . . . . 55

6.4 Reduction of Parametric System via Clustering . . . . . . . . . . 56

6.5 Properties of the Proposed Algorithm . . . . . . . . . . . . . . . 59

6.5.1 Multi-Moment Preservation .............. 59

6.5.2 Stability Preservation . . . . . . . . . . . . . . 62

7 Computational Results $\quad 64$

7.1 Example 1....................... 64

7.2 Example $2 \ldots \ldots \ldots \ldots$. . . . . . . . . . . . . . . . 68

8 Conclusions and Future Work $\quad 73$

8.1 Conclusions ............................ 73

8.2 Future Research . . . . . . . . . . . . . . . . . . . . 74

$\begin{array}{ll}\text { List of References } & 75\end{array}$

Appendix A Mathematical Proof of Lemma $3 \quad 84$

Appendix B Mathematical Proof of Theorem 2 86

Appendix C Mathematical Proof of Theorem $3 \quad 88$

Appendix D Mathematical Proof of Theorem 4 94 


\section{List of Tables}

4.1 Circuit moments. . . . . . . . . . . . . . . . . . 27

7.1 Size, model generation time, MC simulation time and speed-up comparisons $($ example 1$) \ldots \ldots \ldots$. . . . . . . . . . . 68

7.2 Size, model generation time, MC simulation time and speed-up comparisons (example 2) . . . . . . . . . . . . . . . 72 


\section{List of Figures}

3.1 Illustration of projection idea. . . . . . . . . . . . . . . . 14

3.2 Reduction process. . . . . . . . . . . . . . . . 15

5.1 Overall response is obtained by superposing the responses caused by excitation at each input. . . . . . . . . . . . . . 40

5.2 Constructing clusters in $\mathrm{N}$ coupled lines. . . . . . . . . . . . . . 41

7.1 4-Coupled transmission line network (example 1). . . . . . . . . 65

7.2 Sparsity pattern of reduced system matrices $\hat{\mathbf{E}}_{i}$ (left) and $\widehat{\boldsymbol{E}}_{i}$ (right)

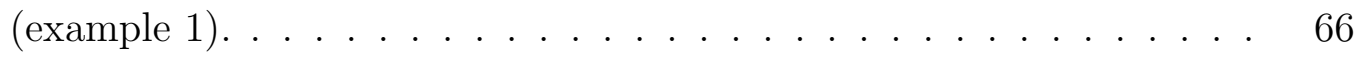

7.3 Statistical analysis for the response at the port \#2 (example 1). . . . 67

7.4 Statistical analysis for the response at the port \#4 (example 1). . . . 67

7.5 24-Coupled transmission line network (example 2). . . . . . . . 69

7.6 Sparsity pattern of reduced system matrices $\hat{\mathbf{E}}_{i}$ (left) and $\widehat{\boldsymbol{E}}_{i}$ (right) (example 2). . . . . . . . . . . . . . . . 70

7.7 Statistical analysis for the response at port \#2. (example 2). . . . . . 71

7.8 Statistical analysis for the response at port \#24. (example 2). . . . . 71 


\section{List of Acronyms}

\begin{tabular}{ll}
\hline Acronyms & Definition \\
\hline \hline CAD & Computer Aided Design \\
CPU & Central Processing Unit \\
DAE & Differential Algebraic Equations \\
EDA & Electronic Design Automation \\
IC & Integrated Circuit \\
KCL & Kirchoff Current Law \\
LHP & Left Half (of the complex) Plane \\
LTI & Linear Time-Invariant \\
MC & Monte Carlo \\
MNA & Modified Nodal Analysis \\
MOR & Model Order Reduction \\
MIMO & Multiple-Input Multiple-Output \\
MSG & Modified Gram-Schmidt \\
MTL & Multi-conductor Transmission Lines \\
ODE & Ordinary Differential Equation \\
PMOR & Parameterized Model Order Reduction \\
PUL & Per Unit Length \\
PRIMA & Passive Reduced-Order Interconnect Macromodel- \\
RHP & ing Algorithm \\
& Right Half (of the complex) Plane
\end{tabular}




\section{List of Symbols}

\begin{tabular}{|c|c|}
\hline Symbols & Definition \\
\hline $\mathbb{N}$ & The field of natural numbers \\
\hline $\mathbb{R}$ & The field of real numbers \\
\hline $\mathbb{C}$ & The field of complex numbers, e.g.: s-plane \\
\hline $\mathbb{R}^{n \times m}$ & The set of real matrices of size $n \times m$ \\
\hline $\mathcal{C}^{n}$ & $n$ differentiable ( $n$-smooth) \\
\hline $\bar{a}$ or $a^{*}$ & The complex conjugate of a complex number $a \in \mathbb{C}$ \\
\hline $\mathbf{A}^{\top}$ & The transpose of matrix $\mathbf{A}$ \\
\hline $\mathbf{A}^{* \top}$ & $\begin{array}{l}\text { The complex-conjugate transpose of complex ma- } \\
\text { trix } \mathbf{A}=\left[a_{i j}\right] \text {, defined as: } \mathbf{A}^{* \top}=\left[a_{j i}^{*}\right]\end{array}$ \\
\hline$\lfloor q / p\rfloor$ & $m=\max \left(\frac{q}{p}\right) \in \mathbb{N}$ \\
\hline $\operatorname{blkdiag}\{\}$ & $\begin{array}{l}\text { blkdiag }\left\{\mathbf{A}_{1}, \ldots, \mathbf{A}_{k}\right\} \text { is a block-diagonal matrix } \\
\text { with the matrix blocks } \mathbf{A}_{1}, \ldots, \mathbf{A}_{n} \text { positioned on } \\
\text { its diagonal, respectively }\end{array}$ \\
\hline \multirow[t]{3}{*}{$\operatorname{span}\{\}$} & Vector space spanned by the given vectors \\
\hline & $\begin{array}{l}\text { The "span" of }\left\{\mathbf{v}_{1}, \ldots, \mathbf{v}_{m}\right\} \in \mathbb{R}^{n \times m} \text { is a set of all } \\
\text { linear combinations of the these vectors forming a } \\
\text { subspace as }\end{array}$ \\
\hline & $\operatorname{span}\left\{\mathbf{v}_{1}, \ldots, \mathbf{v}_{m}\right\}=\left\{\sum_{i=1}^{m} \beta_{i} \mathbf{v}_{i} \forall \beta_{i} \in \mathbb{R}\right\} \subset \mathbb{R}^{n}$ \\
\hline colsp $(\mathbf{A})$ & $\begin{array}{l}\text { Column span (also called range) of matrix } \mathbf{A} \text {. It is } \\
\text { the subspace spanned by the column vectors of } \mathbf{A} \text {. }\end{array}$ \\
\hline
\end{tabular}




\section{Chapter 1}

\section{Introduction}

\section{$1.1 \quad$ Background and Motivation}

Since the beginning of the modern era, the electronic system plays a vital role in daily life. These systems are rapidly improving to provide more complex functionalities. The number of transistors in the integrated circuits doubles about every two years. In 1965, Gordon Moore predicted this trend to follow up to a decade. The growth has continued even hastier in recent decades. The stunning advancements in Very Large-Scale Integration (VLSI) technologies and consistent advancements in Electronic design automation (EDA) tools enable the designs that operate up to gigahertz frequencies with subnanosecond rise time. The constant research to improve Computer-Aided Design (CAD) techniques has been a crucial requirement for designing, verifying, optimizing, and fabricating modern complex circuits. Designers need to repeatedly simulate the designs before implementing them on the physical chip to save the hefty cost of Integrated Circuit (IC) manufacturing. Simulation of electrical designs is to solve a set of mathematical Differential-Algebraic Equations (DAE). The number of equations used for mathematical modeling of modern complex designs grows immensely. The general concept of model-order reduction (MOR) emerged as a response to the need to handle the increasing complexity of the circuits 
and the mathematical problems spawned in their simulations. MOR helps to solve this problem by reducing the number of equations while giving the accurate response of the original system [1].

As the number of ports increases, the MOR model becomes larger and denser. In [2], an algorithm was presented based on clustering technique to alleviate this barrier by creating reduced-order models that are sparse and block-diagonal. Hence, simulation of reduced systems from the proposed method requires less computational time.

Besides time/frequency, other parameters related to geometry/layout, materials features, ambient, or bias conditions can influence the circuit's performance and need to be considered in the mathematical models for practical designs. Designers must further analyze the influence of these design parameters on the circuit's behavior to ensure the satisfaction of the design specifications. This requires repeated simulations for different values of design parameters that can be computationally expensive. The Parameterized Model Order Reduction (PMOR) is a means to address this issue. The reduction of the model is based on the series expansion of the parameter dependence followed by single or multi-parameter reduction. However, the resulting parametric reduced models can be prohibitively CPU extensive for the system with many ports. This thesis develops a novel, efficient, and accurate PMOR approach by adopting the clustering algorithm in [2] to the context of parameterized circuits.

\subsection{Objectives and Contributions}

The contribution introduced in this thesis targets the application of PMOR to the parameterized systems with a large number of input/output ports. The coupled multi-conductor interconnect networks with several design parameters are pertinent examples for this class of multiport networks. This thesis developed a novel algorithm 
for constructing the parametric reduced model for massively coupled large parametric interconnects networks. The proposed method preserves the stability of the original circuit throughout the entire design space. It also preserves the first $(m)$ blockmoments of the original multiport circuits.

The proposed method provides an effective means for the efficient statistical analysis of any general parameterized multiport circuit, and its application is not limited to the interconnect problems.

\subsection{Organization of the Thesis}

The rest of this thesis is organized as follows.

- Chapter 2 provides an introduction to the Modified Nodal Analysis (MNA) for the formulation of electrical circuits and reviews the key notions for linear circuits such as transfer functions, poles. It also introduces the essential physical characteristics of linear circuits, i.e., stability and passivity.

- Chapter 3 reviews the concept of model-order reduction and its application in the context of circuit simulation. It then presents an overview on the construction of reduction projection operator, the Krylov subspace-based reduction algorithm, such as PRIMA.

- Chapter 4 discusses the application of the MOR algorithms introduced in Chapter 3 to the domain of parametric circuits to track the variations in the circuit response due to variations in a selected set of design parameters.

- Chapter 5 describes the fundamental idea of the clustering scheme for the reduction of multi-port systems, such as massively coupled interconnect structures. 
- Chapter 6 presents the main contributions in this thesis. It describes the application of PMOR to the domain of multi-variate multi-port systems. It focuses on the parametric reduction of coupled multi-conductor interconnect networks with several design parameters.

- Chapter 7 presents examples to demonstrate the accuracy and efficiency of the proposed method.

- Chapter 8 provides closing remarks and discusses possible future work based on the findings of this thesis. 


\section{Chapter 2}

\section{Formulation of Circuit Equations}

Rapid growth in VLSI technologies has led to large and complex designs that support wide frequency bandwidth with cutoff frequencies over tens of $\mathrm{GHz}$ and pulses of picosecond rise times. Modern designs require advanced Electronic Design Automation (EDA) tools to solve complex and large systems. An essential capability necessary for all the EDA tools is the automated circuit formulation. This section presents the Modified Nodal Analysis (MNA) approach used by virtually all commercial circuit simulators to represent general circuits with lumped elements.

\subsection{MNA Matrix Equations}

MNA is an extension of the nodal analysis method in standard circuit theory [3]. Its advantages lie first in its ability to handle all types of circuit components and that its formulation process is highly suited for automation. A general circuit comprises lumped components such as resistor, capacitor, inductor, voltage source, etc. Constructing the MNA formulation is usually done on an element-by-element basis. The main idea behind MNA is to create stamps of these components. The "Component Stamping" is referred to the process of placing the circuit components in the corresponding MNA matrices ( $\mathbf{C}$ and $\mathbf{G}$ ). The stamping process converts components into 
numerical entries in the above MNA matrices, yielding large matrix equations consisting of first-order differential and algebraic equations (DAE). Construction of the circuit equations in MNA formulation is achieved through the following steps [4-6].

- Considering node voltages as the unknowns;

- Expressing the currents in the circuit elements in terms of the node voltages;

(1) Considering the admittance form of constitutive relation of the circuit elements;

(2) Casting a special representation for the elements which do not have explicit representation in an admittance form (e.g., voltage sources);

(3) Casting a special representation for the elements whose constitutive relation requires integration in the time-domain (e.g., inductors);

(4) Casting the charge or flux based formulation for nonlinear capacitors or inductors, respectively;

- Writing the Kirchoff Currents Law (KCL) at each node;

- Considering node voltages, current in the branch (for items (2) and (3)), and the charge or flux (for item (4)) as the unknowns in the formulation;

- The MNA circuit equations will be solved to obtain these circuit unknowns;

This repetitive process of placing stamps in MNA matrices can be readily automated.

\subsubsection{MNA Formulation of Linear Circuits}

By using the above steps, a general Linear Time-Invariant (LTI) system is described using the Modified Nodal Analysis (MNA) formulation as [4-6]

$$
\begin{aligned}
\mathbf{G x}(t)+\mathbf{C} \frac{d \mathbf{x}(t)}{d t} & =\mathbf{B u}(t) \\
\mathbf{y}(t) & =\mathbf{L x}(t)
\end{aligned}
$$


where

- $\mathbf{x}(t) \in \mathbb{R}^{n \times 1}$ is a vector of node voltages appended by currents in inductors, independent and dependent voltage sources,

- $\mathbf{G} \in \mathbb{R}^{n \times n}$ is a matrix representing the memory-less components of the circuit such as resistors,

- $\mathbf{C} \in \mathbb{R}^{n \times n}$ is a matrix representing the components dependent on the changes in node voltages (components with memory) such as capacitors,

- $\mathbf{B} \in \mathbb{R}^{n \times n_{i n}}$ is the input selection matrix,

- $\mathbf{u}(t) \in \mathbb{R}^{n_{i n} \times 1}$ is a vector representing the independent voltage and current sources.

- $\mathbf{L} \in \mathbb{R}^{n_{\text {out }} \times n}$ is the output selection matrix,

- $\mathbf{y}(t) \in \mathbb{R}^{n_{\text {out }}}$ contains the outputs that are the selections of the entries from $\mathbf{x}(t)$.

The DAE in (2.1) should be solved using properly selected numerical integration techniques through marching in time to obtain the trajectory of the circuit response $\mathbf{x}(t)$ for a given initial condition. This process is called the transient simulation.

Applying Laplace transformation to the time-domain equations (2.1) yields a frequency-domain realization for the LTI systems as

$$
\begin{aligned}
\mathbf{G X}(s)+\mathbf{C} s \mathbf{X}(s) & =\mathbf{B U}(s) \\
\mathbf{Y}(s) & =\mathbf{L X}(s)
\end{aligned}
$$

where the Laplace variable $s$ is a complex frequency, $\mathbf{X}(s) \in \mathbb{C}^{n}, \mathbf{U}(s) \in \mathbb{C}^{n_{\text {in }}}$, $\mathbf{Y}(s) \in \mathbb{C}^{n_{\text {out }}}$. 


\subsection{Properties of MNA Matrices}

The circuit matrices $\mathbf{C}, \mathbf{G}$, and $\mathbf{B}$ from MNA formulation can be obtained as blockstructured matrices with specific properties as shown in (2.3). These structural properties ensure specific physical characteristics for the circuit models. For the circuit variables partitioned as $\mathbf{x}=[\mathbf{v}, \mathbf{i}]^{\top}$, the circuit matrices that are partitioned accordingly will have the following block structure

$$
\mathbf{G}=\left[\begin{array}{cc}
\mathbf{F} & \mathbf{E} \\
& \\
\mathbf{E}^{t} & 0
\end{array}\right] \quad \mathbf{C}=\left[\begin{array}{cc}
\mathbf{C}_{a} & 0 \\
0 & -\mathbf{C}_{b}
\end{array}\right]
$$

where $\mathbf{F}, \mathbf{C}_{\mathbf{a}}$ and $\mathbf{C}_{\mathbf{b}}$ hold the information of resistance, capacitance, and inductance

of the circuit. Note that the stamps are created in such a way that $\mathbf{C}_{\mathbf{a}} \geq 0$ and $\mathbf{C}_{b} \geq 0$ (positive semidefinite), for every entry in $\mathbf{E}$ as $e_{i j}$ we have $e_{i j} \in\{-1,0,1\}$, and for symmetric (passive) circuit $\mathbf{F} \geq 0$. Hence, the matrix $\mathbf{G}$ is always symmetrical for the passive circuits. However, for the active circuits, G loses its symmetry. In this thesis, all the circuits discussed are passive circuits. In addition, overall, MNA matrices are sparse, wherein most of the entries in the MNA matrices are zero with only a few nonzero entries.

These are important properties that will be further considered in the following sections.

\subsection{Circuit transfer function}

Laplace-domain realization in (2.2) allows defining the transfer function for LTI systems as a complex matrix-valued function relating the inputs to outputs as

$$
\mathbf{Y}(s)=\mathbf{H}(s) \mathbf{U}(s)
$$


where

$$
\mathbf{H}(s)=\mathbf{L}(\mathbf{G}+s \mathbf{C})^{-1} \mathbf{B} \in \mathbb{C}^{n_{\text {out }} \times n_{\text {in }}}
$$

The circuit transfer function in (2.5) can be equivalently rewritten as

$$
\begin{aligned}
\mathbf{H}(s) & =\mathbf{L}\left[\mathbf{G}\left(\mathbf{I}_{n}+\mathbf{G}^{-1} \mathbf{C} s\right)\right]^{-1} \mathbf{B} \\
& =\mathbf{L}\left(\mathbf{I}_{n}+\mathbf{G}^{-1} \mathbf{C} s\right)^{-1} \mathbf{G}^{-1} \mathbf{B} \\
& =\mathbf{L}\left(\mathbf{I}_{n}-s \mathbf{A}\right)^{-1} \mathbf{R}
\end{aligned}
$$

where $\mathbf{I}_{n}$ is an $n \times n$ unity matrix and

$$
\begin{aligned}
& \mathbf{A}=-\mathbf{G}^{-1} \mathbf{C} \in \mathbb{R}^{n \times n} \\
& \mathbf{R}=\mathbf{G}^{-1} \mathbf{B} \in \mathbb{R}^{n \times n_{i n}}
\end{aligned}
$$

\section{System poles}

Poles of a linear system are the singularities of its transfer function (2.6). Given a single-input single-output circuit, its transfer function is a complex-valued rational function as $H(s)=\frac{N(s)}{D(s)}$ where $N(s)$ and $D(s)$ are polynomials with real coefficients. The finite poles of $H(s)$ are the roots of its denominator $D(s)$, counted with appropriate multiplicity. A transfer function can also have a pole at infinity when degree of $N(s)>$ degree of $D(s)$. Poles of a matrix-valued transfer function are a collection of the poles for all its entries. This is illustrated for a network with three inputs and two outputs as follows.

$$
\mathbf{H}(s)=\left[\begin{array}{lll}
h_{11}(s) & h_{12}(s) & h_{13}(s) \\
h_{21}(s) & h_{22}(s) & h_{23}(s)
\end{array}\right] \in \mathbb{C}^{2 \times 3}
$$


where

Poles of $\mathbf{H}(s)=$ Poles of $h_{11}(s) \cup$ Poles of $h_{12}(s) \cup \cdots$

$\cup$ Poles of $h_{22}(s) \cup$ Poles of $h_{23}(s)$

According to the above definition, the circuit poles.

The relationship between the circuit poles as the singularities of its transfer function $\mathbf{H}(s)$ (2.6) and the roots of a polynomial,

$$
\operatorname{det}\left(\mathbf{I}_{n}-\lambda_{i} \mathbf{A}\right)=0
$$

is explored in Proposition 1. It is also shown that the circuit poles can be determined through Eigendecomposition of the circuit matrix $\mathbf{A}$ as [7]

$$
\mathbf{A}=\mathbf{P} \lambda \mathbf{P}^{-1}
$$

where $\mathbf{P}$ and $\boldsymbol{\lambda}$ are referred to as eigenvector matrix and eigenvalue matrix of $\mathbf{A}$, respectively.

Proposition 1 (Circuit poles). The poles of a circuit in (2.1) are the reciprocal of the nonzero eigenvalues of its matrix $\mathbf{A}$ in (2.7a)

Proof. let the nonzero eigenvalues of matrix A from its Eigendecomposition (2.11) be denoted as

$$
\boldsymbol{\lambda}(\mathbf{A})=\left\{\lambda_{i} \mid \operatorname{det}\left(\mathbf{I}_{n}-\lambda_{i} \mathbf{A}\right)=0, \lambda_{i} \neq 0\right\} .
$$


By substituting (2.11) in (2.6), we have

$$
\begin{aligned}
\mathbf{H}(s) & =\mathbf{L P}\left(\mathbf{I}_{n}-s \boldsymbol{\lambda}\right)^{-1} \mathbf{P}^{-1} \mathbf{R} \\
& =\mathbf{L P}\left[\begin{array}{ccc}
\frac{1}{1-s \lambda_{1}} & & \\
& \ddots & \\
& & \\
& & \frac{1}{1-s \lambda_{n}}
\end{array}\right] \mathbf{P}^{-1} \mathbf{R}
\end{aligned}
$$

From (2.13), the poles (singularities of $\mathbf{H}(s)$ ) are obtained as the solutions for $1-p_{i} \lambda_{i}=0$, for $i=1,2, \ldots, n$. Hence, the poles are $p_{i}=\frac{1}{\lambda_{i}}$, for $i=1,2, \ldots, n$. This concludes the proof.

\subsection{Stability of Linear Designs}

The stability is an important characteristics defined based on the dynamic systems' trajectory behavior undergone a slight disturbance in the proximity of its equilibrium point. If the response trajectories do not go to infinity due to any slight disturbances, the system is considered as stable; otherwise, it is known as unstable [8]. For a linear dynamic system, the locations of its poles determine its stability considering the following theorem 1 .

Theorem 1 (Stability). A stable system has all its poles on the close left half-plane of the complex plane; all pure imaginary poles have multiplicity one. An asymptotically stable system has all its poles on the open left half-plane in the complex plane 


\section{Chapter 3}

\section{Model Order Reduction Techniques}

Simulation of practical dynamic systems is, in general, CPU-expansive due to their large size and complexity. The concept of Model Order Reduction (MOR) has been spawned in system theory and mathematics. Then, MOR was applied to the problems in control engineering, where real-time simulation of the systems is essential. Successful extension of MOR idea to different problems has proven MOR to be an effective tool in reducing the computational complexity of simulating large systems [1,9-15]. The recent development of MOR techniques has been stimulated by their popularity and success in broader fields applications, including microelectronics [16-20], high-speed and RF circuits [21-25], uncertainty quantification [21, 26-29], electromagnetic [30,31], thermal analysis [32], mechanical, biomedical, civil, and aerospace engineering [33-35].

This chapter presents the fundamentals of the Model Order Reduction and its application in circuit simulation and related notation. A complete account of MOR techniques and applications is too vast to be covered in one chapter. Rather, this chapter reviews the techniques pertained to the subject of this thesis, and the associated difficulties. Section 3.1 introduces the idea of MOR as a general concept 


\subsection{The Concept of MOR}

MOR is a natural approach to reduce the simulation time of large complex systems. The central idea behind MOR is that a large system with well defined input-output can be approximated to produce almost the same response while having a smaller number of equations to solve. By reducing the order of the model, it is possible to drastically reduce the computation time. This may be considered as an accuracy vs. efficiency trade-off. However, in most applications, the system can be reduced to a certain extent to minimize the CPU-cost without noticeable accuracy degradation.

Model-Order Reduction (MOR) for linear systems is a well-established area, and a rich body of literature is available on the subject $[1,13,15,20,36-38]$. The projectionbased approach to reduce the linear circuits is presented in the next section.

\subsection{MOR for Linear Circuits}

The MNA formulation for general linear circuits is previously explained in Chapter 2. The general linear MNA matrix equation is presented in (2.1) and is repeated here for reference.

$$
\begin{aligned}
\mathbf{G x}(t)+\mathbf{C} \frac{d \mathbf{x}(t)}{d t} & =\mathbf{B u}(t) \\
\mathbf{y}(t) & =\mathbf{L} \mathbf{x}(t)
\end{aligned}
$$

where $\mathbf{x}(t) \in \mathbb{R}^{n}$ is the vector of $n$ unknowns. The change in the state of variables $\mathbf{x}(t)$ with time creates the system trajectory. Finding the response trajectory of a dynamic system is the intent of simulation. The key idea in MOR is reducing the number of system equations and hence condensing the number of system variables. The order reduction can be achieved by projecting the system trajectory $\mathbf{x}(\cdot)$ from 
the original n-dimensional space to a smaller subspace. As a result of projection using a projection operator matrix $\mathbf{V}$, the size of $\mathbf{x}(\cdot)$ is drastically reduced as illustrated in Fig. 3.1.

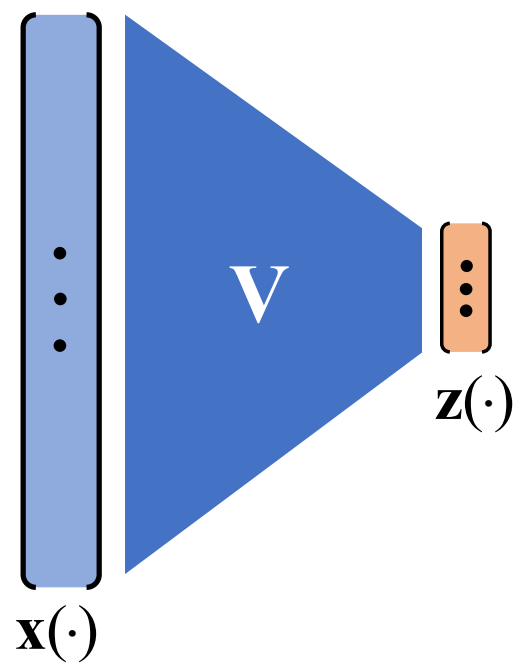

Figure 3.1: Illustration of projection idea.

Given a projection vector $\mathbf{V} \in \mathbb{R}^{n \times q}$, the number of unknowns in $\mathbf{x}(\cdot)$ is truncated from $n$ variables to $q$ in the new vector $\mathbf{z}(\cdot) \in \mathbb{R}^{q}(q \ll n)$, where it is

$$
\mathbf{x}(t)=\mathbf{V} \mathbf{z}(t)
$$

Next, by using the variable change in (3.2), from (3.1), we get

$$
\begin{array}{r}
\mathbf{G V z}(t)+\mathbf{C V} \frac{d \mathbf{z}(t)}{d t}=\mathbf{B u}(t) \\
\mathbf{y}(t)=\mathbf{L V z}(t)
\end{array}
$$

The residual error caused by such a variable change using the projection matrix is

$$
\boldsymbol{\eta}(t)=\mathbf{G V z}(t)+\mathbf{C V} \frac{d \mathbf{z}(t)}{d t}-\mathbf{B u}(t)
$$


Following Galerkin projection scheme [15], by pre-multiplying $\mathbf{V}^{\top}$ to equation (3.4) gives the minimum error [39]

$$
\mathbf{V}^{\top} \boldsymbol{\eta}(t)=\mathbf{V}^{\top} \mathbf{G} \mathbf{V} \mathbf{z}(t)+\mathbf{V}^{\top} \mathbf{C V} \frac{d \mathbf{z}(t)}{d t}-\mathbf{V}^{\top} \mathbf{B} \mathbf{u}(t)=0
$$

The new reduced model can be represented as

$$
\begin{aligned}
\mathbf{G}_{r} \mathbf{z}(t)+\mathbf{C}_{r} \frac{d \mathbf{z}(t)}{d t} & =\mathbf{B}_{r} \mathbf{u}(t) \\
\mathbf{y}(t) & =\mathbf{L}_{r} \mathbf{z}(t)
\end{aligned}
$$

where

$$
\begin{aligned}
\mathbf{G}_{r} \triangleq \mathbf{V}^{\top} \mathbf{G} \mathbf{V} \in \mathbb{R}^{q \times q} & \mathbf{C}_{r} \triangleq \mathbf{V}^{\top} \mathbf{C V} \in \mathbb{R}^{q \times q} \\
\mathbf{B}_{r} \triangleq \mathbf{V}^{\top} \mathbf{B} \in \mathbb{R}^{q \times n_{\text {in }}} & \mathbf{L}_{r} \triangleq \mathbf{L V} \in \mathbb{R}^{n_{\text {out }} \times q}
\end{aligned}
$$

Figure 3.2 illustrates the reduction process by applying the projection matrix to reduce the circuit equation.
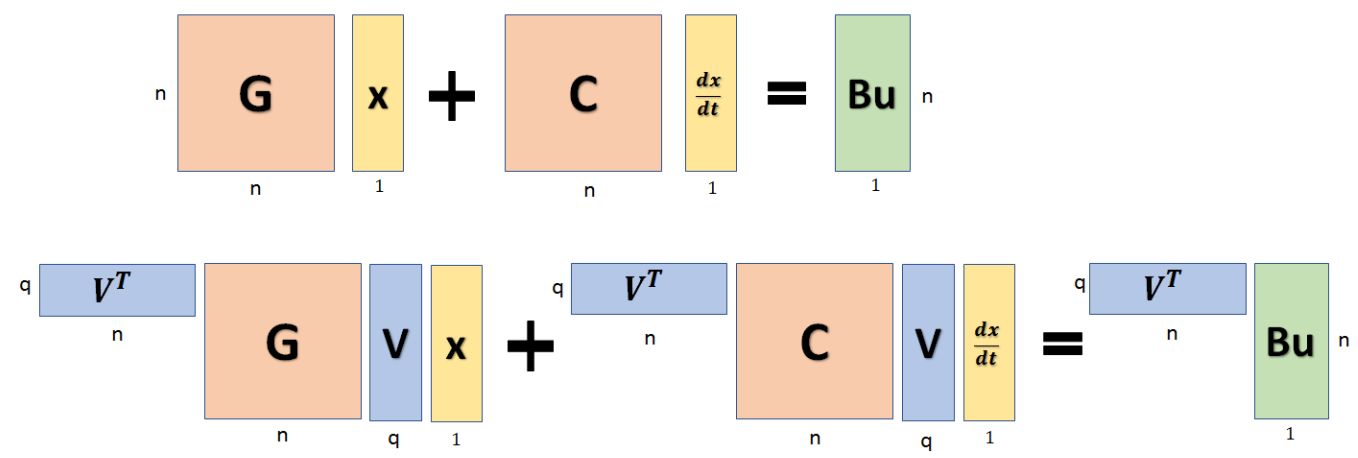

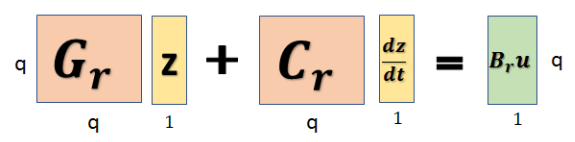

Figure 3.2: Reduction process. 
The essential features of the original system are preserved in the reduction process and passed to the resulting reduced model. It guarantees that the reduced model in (3.6) ideally provides a sufficiently accurate approximation for the circuit response $\mathbf{y}(t)$.

\subsection{Construction of Projection Matrix}

The projection-based framework described in Section 3.2 is an effective reduction strategy that has been vastly explored in literature for linear systems $[1,13,15,20,40-$ 45]. It is constituted based on the idea of using a reduction projection matrix $\mathbf{V} \in$ $\mathbb{R}^{n \times q}$. The construction of the projection matrix is an important stage in the orderreduction process. MOR methods are indeed differentiated based on the technique employed to obtain their projection operator matrix.

Finding suitable projection matrices is the main task in any MOR method. The following section will elaborate on the construction of $\mathbf{V}$, as related to the subject of this thesis.

\subsubsection{Krylov-Subspace Based Methods}

Consider the circuit transfer function given in (2.5) as

$$
\mathbf{H}(s)=\mathbf{L}(\mathbf{G}+s \mathbf{C})^{-1} \mathbf{B}
$$

With a reasonable assumption that the matrix $(\mathbf{G}+s \mathbf{C})$ is non-singular except at a finite number of points, $s_{o}$ as an expansion point is chosen such that $\left(\mathbf{G}+s_{o} \mathbf{C}\right)$ is non-singular. The transfer function (3.7) can be expanded in the Taylor series in the vicinity of $s_{o}$ as 


$$
\mathbf{H}(s)=\mathbf{L} \sum_{j=0} \mathbf{M}_{j}\left(s_{o}\right)\left(s-s_{o}\right)^{j}
$$

where the coefficient of $\left(s-s_{o}\right)^{j}$,

$$
\mathbf{M}_{j}\left(s_{o}\right)=\left.\frac{1}{j !} \frac{\partial^{j} \mathbf{H}(s)}{\partial s^{j}}\right|_{s=s_{o}}
$$

is called the $j$-th (block) moment of the circuit at $s_{o}$, given by [20]

$$
\mathbf{M}_{j}\left(s_{o}\right) \triangleq \mathbf{A}^{j} \mathbf{R}
$$

where

$$
\begin{array}{ll}
\mathbf{R} \triangleq\left(\mathbf{G}+s_{o} \mathbf{C}\right)^{-1} \mathbf{B} & \in \mathbb{R}^{n \times n_{i n}} \\
\mathbf{A} \triangleq-\left(\mathbf{G}+s_{o} \mathbf{C}\right)^{-1} \mathbf{C} & \in \mathbb{R}^{n \times n}
\end{array}
$$

It is to be noted that matrices $\mathbf{R}$ and $\mathbf{A}$ as given in (2.7) can be obtained from (3.9b) and $(3.9 \mathrm{c})$ for $s_{o}=0$, respectively.

Moreover, the moments for the transfer function can be obtained from (3.9) as

$$
\mathcal{M}_{i}\left(s_{o}\right)=\mathbf{L M}_{j}\left(s_{o}\right) \quad \in \mathbb{R}^{n_{\text {out }} \times n_{\text {in }}}
$$

The Krylov-Subspace-based MOR methods construct an orthogonal projection matrix

$$
\mathbf{V}^{\top} \mathbf{V}=\mathbf{I}_{q}
$$

such that the $q$ columns of $\mathbf{V}$ span the space formed by the $m=\left\lfloor q / n_{\text {in }}\right\rfloor$ block-moment 
matrices as

$$
\operatorname{colsp} \mathbf{V}=\mathcal{K} \boldsymbol{r}(\mathbf{A}, \mathbf{R}, m)=\operatorname{span}\left\{\mathbf{R}, \mathbf{A R}, \ldots, \mathbf{A}^{(m-1)} \mathbf{R}\right\}
$$

The moments can be explicitly calculated using the following recursion

$$
\mathbf{M}_{j}=\mathbf{A}^{j} \mathbf{M}_{j-1}, \quad \mathbf{M}_{0}=\mathbf{R} .
$$

It is important to note that the sequence $\mathbf{M}_{j}=\mathbf{A}^{j} \mathbf{M}_{j-1}$ for $j=1, \ldots$ converges to the eigenvector corresponding to the largest eigenvalue of $\mathbf{A}$. Therefore, the matrix of block moments $\left\{\mathbf{R}, \mathbf{A R}, \ldots, \mathbf{A}^{(m-1)} \mathbf{R}\right\}$ can quickly become ill-conditioned after first few moments. This fact imposes a limiting factor for the number of projection bases and the attainable order for the reduced model.

\subsubsection{Arnoldi Algorithm}

The problem of convergence of higher-order moments can be solved by the implicit moment matching technique. The Arnoldi algorithm is an example of an efficient method for computing $\mathbf{V}[46,47]$. It is established based on the following property.

Proposition 2. If $\mathbf{V}$ is the orthogonal basis of $K_{r}(\mathbf{A}, \mathbf{R}, m)$ then $\mathbf{A V}=\mathbf{V H}$. Where $\mathbf{H}$ is an upper-Hessenberg matrix with zero entries below the first subdiagonal and $\mathbf{A}$ is given in $(3.9 \mathrm{c})$.

By using the above Proposition 2, the Arnoldi algorithm computes the basis of the Krylov subspace without explicitly forming the moments. A sample Pseudo-code demonstrating the steps of the Arnoldi process is presented in Algorithm 1.

The above algorithm is for the single input circuits provided as an example. However, obtaining the Krylov basis for the general multi-input circuits requires slight modifications. One can refer to [20] for the general Arnoldi algorithm. 


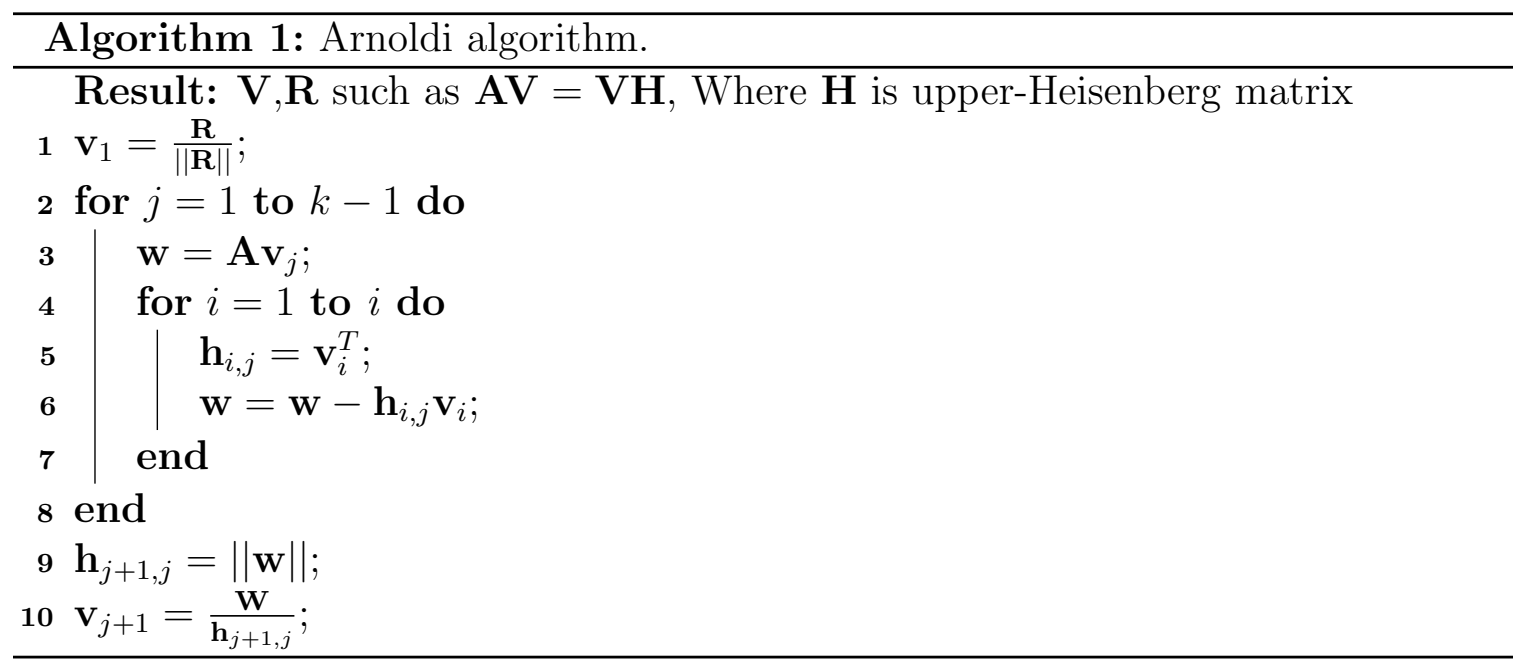

The following section provides an overview of PRIMA, a well-established Krylovsubspace based reduction reduction algorithm using the Arnoldi process.

\subsubsection{PRIMA}

PRIMA is the abbreviation of Passive Reduced-Order Interconnect Macromodeling Algorithm [48]. The basic idea of this algorithm is to use the basis of Krylov subspace, calculated through the Arnoldi algorithm, with some minor manipulation when constructing the MNA matrices. The resulting MOR is guaranteed to be stable and passive for all passive circuits. The important modification introduce by PRIMA is negating the rows of MNA matrices associated with the inductances and voltage sources, as shown below.

$$
\mathbf{G}=\left[\begin{array}{cc}
\mathbf{F} & \mathbf{E} \\
-\mathbf{E}^{T} & 0
\end{array}\right] \quad \mathbf{C}=\left[\begin{array}{cc}
\mathbf{C}_{a} & 0 \\
0 & \mathbf{C}_{b}
\end{array}\right] \quad \mathbf{x}=\left[\begin{array}{c}
\mathbf{v} \\
-\mathbf{i}
\end{array}\right]
$$


The modified $\mathbf{G}$ and $\mathbf{C}$ matrices hold the following properties

$$
\mathbf{C} \geq 0 \quad\left(\mathbf{G}^{T}+\mathbf{G}\right) \geq 0
$$

The projection matrix from Arnoldi process $\mathbf{V}$ and the negated MNA matrices are used to generate the PRIMA reduced order model as shown in (3.6). The PRIMA reduced models for the originally passive networks are passive and stable.

\subsection{Summary and Discussions}

First, the reduced model of active circuit construct using PRIMA is not always stable. PRIMA exploits the structural properties of the passive system matrices; these properties may lose when working with an active circuit. A novel algorithm is proposed in [49], that solves this problem. Second, when the system is large or with many ports, the reduced system matrices generated using the Arnoldi algorithm are dense, making the simulation of the resulting reduced model more CPU expansive than the original system. In Chapter 5, a new algorithm explains that can preserve the sparsity of the reduced model.

It is to be noted that the reduction techniques presented so far did not account for the design parameters such as those related to ambient, bias conditions, geometry/layout, materials features, or other fabrication process parameters. These parameters can noticeably influence circuits' performance and hence are considered in the mathematical models for designs. The next chapter is devoted to reviewing the model order reduction methods for parametrized circuits. 


\section{Chapter 4}

\section{MOR for Parametric Circuits}

The previous two chapters dealt with the model order reduction of large systems whose responses only depend on time " $t$ " (or correspondingly on frequency " $s$ "). However, in practical designs, other parameters related to geometry/layout, materials features, ambient, or bias conditions can influence the circuit's performance and need to be considered. Therefore, designers must further analyze the influence of these design parameters on the circuit's behavior to ensure the satisfaction of the design specifications. This leads to design tasks such as various sensitivity analyses, simulation-based design optimization, and "design space exploration" and Monte Carlo (MC) analyses. In the context of circuit simulation, "design space exploration" is commonly referred to as "parameter sweep," which is the notion of the repeated stimulation of circuits with different values for the selected set of design parameters, that is, in general, computationally cumbersome. The repeated simulations of large circuits at numerous points in the designated design parameters space naturally lead to high computational complexity.

Capitalized on the savings achieved by using reduced-order models, Parametric Model Order Reduction (PMOR) methods [29, 30, 45, 50-59] have been developed in the literature to remedy the high CPU-cost problem in the simulation of parametric circuits under different values for the design parameters. 
This section focuses on parametric circuits, i.e., designs which have more than one design parameter. It aims to show how to incorporate the design parameters in the circuit representation and to review the role that MOR techniques play in reducing the computational cost associated with the idea of "design space explorations."

\subsection{General Formulation of Parametric Circuits}

Chapter 2 presented MNA matrix equations describing general linear circuits where the response depended only on time/frequency. This section focus on the mathematical representation for circuits with multiple parameters. Consider a time-invariant circuit with $n$ circuit variables that are dependent on time (hence, frequency) and on $\mu$ additional parameters collected in vector

$$
\boldsymbol{\lambda}=\left\{\lambda_{1}, \lambda_{2}, \ldots, \lambda_{\mu}\right\} \in \boldsymbol{\Lambda} \subset \mathbb{R}^{\mu}
$$

These parameters may be related to geometry (e.g., width or separation of traces), materials (e.g., relative permittivity), or ambient (e.g., temperature). The mathematical formulation for such parametric circuits is obtained through the Modified Modal Analysis (MNA) approach as

$$
\begin{aligned}
\mathbf{C}(\boldsymbol{\lambda}) \frac{d \mathbf{x}(t, \boldsymbol{\lambda})}{d t}+\mathbf{G}(\boldsymbol{\lambda}) \mathbf{x}(t, \boldsymbol{\lambda}) & =\mathbf{B u}(t) \\
\mathbf{y}(t, \boldsymbol{\lambda}) & =\mathbf{L x}(t, \boldsymbol{\lambda})
\end{aligned}
$$

where $\mathbf{x}(t, \boldsymbol{\lambda})) \in \mathbb{R}^{n}$ is $n$ circuit variables dependant on time and parameters, $\mathbf{C}(\boldsymbol{\lambda}), \mathbf{G}(\boldsymbol{\lambda}) \in \mathbb{R}^{n \times n}$ are parameter-dependent matrices that carry the stamps of memory and memoryless elements, respectively. $\mathbf{B} \in \mathbb{R}^{n \times n_{\text {in }}}$ and $\mathbf{L} \in \mathbb{R}^{n_{\text {out }} \times n}$ are the input and output selector matrices, respectively. 
The frequency domain representation of the circuit in $(4.2 \mathrm{a})$ is

$$
\begin{aligned}
\mathbf{E}(s, \boldsymbol{\lambda}) \mathbf{X}(s, \boldsymbol{\lambda}) & =\mathbf{B U}(s) \\
\mathbf{Y}(s, \boldsymbol{\lambda}) & =\mathbf{L X}(s, \boldsymbol{\lambda})
\end{aligned}
$$

where $\mathbf{X}(s, \boldsymbol{\lambda}) \in \mathbb{C}^{n}$ is the parameter-dependent circuit unknowns in frequency domain, $s \in \mathbb{C}$ is the complex frequency, and

$$
\mathbf{E}(s, \boldsymbol{\lambda}) \triangleq(\mathbf{G}(\boldsymbol{\lambda})+s \mathbf{C}(\boldsymbol{\lambda})) \in \mathbb{C}^{n \times n}
$$

is the system descriptor matrix.

From a general perspective, one can also treat the frequency $f$ as a (non-stochastic) parameter by hypothetically denoting it as $\lambda_{i}$, which allows shortening equations by dropping $s$. We will follow this form treatment for the following equations in the rest of this chapter for brevity.

The descriptor matrix $\mathbf{E}\left(\lambda_{1}, \ldots, \lambda_{\mu}\right)$ is, in general, a complex non-affine function of the designated parameters $\lambda_{1}, \ldots, \lambda_{\mu}$. The first step in the direction of parametric model order reduction is to represent the descriptor matrix in some appropriate approximation in the affine form, i.e.,

$$
\mathbf{E}(\boldsymbol{\lambda})=\mathbf{E}_{0}+\sum_{i} \Delta \lambda_{i} \mathbf{E}_{i}+\sum_{h, k} \Delta \lambda_{h} \Delta \lambda_{k} \mathbf{E}_{h, k}+\sum_{h, k, j} \Delta \lambda_{h} \Delta \lambda_{k} \Delta \lambda_{j} \mathbf{E}_{h, k, j}+\cdots
$$

where $\Delta \lambda_{i}:=\lambda_{i}-\bar{\lambda}_{i}$.

Polynomial interpolation and power series expansions such as Chebyshev polynomial and Taylor series are means to accomplish this. The Taylor series expansion for parametric $\mathbf{E}(\boldsymbol{\lambda})$ in (4.4) at an expansion point in the parameter space as $\bar{\lambda}=\left(\bar{\lambda}_{1}, \ldots, \bar{\lambda}_{\mu}\right)$ yields 


$$
\begin{array}{r}
\mathbf{E}(\boldsymbol{\lambda})=\mathbf{E}(\overline{\boldsymbol{\lambda}})+\sum_{i} \frac{\Delta \lambda_{i}}{\bar{\lambda}_{i}}\left(\left.\bar{\lambda}_{i} \frac{\partial \mathbf{E}(\boldsymbol{\lambda})}{\partial \lambda_{i}}\right|_{\overline{\boldsymbol{\lambda}}}\right)+\sum_{h, k} \frac{\Delta \lambda_{h}}{\bar{\lambda}_{h}} \frac{\Delta \lambda_{k}}{\bar{\lambda}_{k}}\left(\left.\bar{\lambda}_{h} \bar{\lambda}_{k} \frac{\partial^{2} \mathbf{E}(\boldsymbol{\lambda})}{\partial \lambda_{h} \lambda_{k}}\right|_{\overline{\boldsymbol{\lambda}}}\right)+ \\
\sum_{h, k, j} \frac{\Delta \lambda_{h}}{\bar{\lambda}_{h}} \frac{\Delta \lambda_{k}}{\bar{\lambda}_{k}} \frac{\Delta \lambda_{j}}{\bar{\lambda}_{j}}\left(\left.\bar{\lambda}_{h} \bar{\lambda}_{k} \bar{\lambda}_{j} \frac{\partial^{3} \mathbf{E}(\boldsymbol{\lambda})}{\partial \lambda_{h} \lambda_{k} \lambda_{j}}\right|_{\overline{\boldsymbol{\lambda}}}\right)+\cdots
\end{array}
$$

In practical setups, the nominal values for the parameters can be used as an expansion point $\overline{\boldsymbol{\lambda}}$, also, a relative (normalized) variations as $\frac{\Delta \lambda_{i}}{\bar{\lambda}_{i}}$ is more useful to work with rather than absolute variations $\Delta \lambda_{i}$.

For brevity in presenting (4.6), let us consider the following shorthand notations for the system matrices $\tilde{\mathbf{E}}_{i}$ and parameters set $\tilde{\lambda}_{i}$ in (4.7) and (4.8), respectively.

$$
\begin{aligned}
& \tilde{\mathbf{E}}_{i}: \begin{cases}\tilde{\mathbf{E}}_{0}:=\mathbf{E}(\overline{\boldsymbol{\lambda}})=\mathbf{G}(\overline{\boldsymbol{\lambda}})+\bar{s} \mathbf{C}(\overline{\boldsymbol{\lambda}}) & \\
\tilde{\mathbf{E}}_{1}:=\left.\bar{\lambda}_{i} \frac{\partial \mathbf{E}(\boldsymbol{\lambda})}{\partial \lambda_{i}}\right|_{\overline{\boldsymbol{\lambda}}}, & \mathrm{i}=1, \ldots, \mu \\
\tilde{\mathbf{E}}_{2}:=\left.\bar{\lambda}_{h} \bar{\lambda}_{k} \frac{\partial^{2} \mathbf{E}(\boldsymbol{\lambda})}{\partial \lambda_{h} \lambda_{k}}\right|_{\overline{\boldsymbol{\lambda}}}, & h, k=1, \ldots, \mu \\
\tilde{\mathbf{E}}_{3}:=\left.\bar{\lambda}_{h} \bar{\lambda}_{k} \bar{\lambda}_{j} \frac{\partial^{3} \mathbf{E}(\boldsymbol{\lambda})}{\partial \lambda_{h} \lambda_{k} \lambda_{j}}\right|_{\overline{\boldsymbol{\lambda}}} & h, k, j=1, \ldots, \mu \\
\vdots & \end{cases} \\
& \tilde{\lambda}_{i}: \begin{cases}\tilde{\lambda}_{1}:=\frac{\Delta \lambda_{i}}{\bar{\lambda}_{i}}, & \mathrm{i}=1, \ldots, \mu \\
\tilde{\lambda}_{2}:=\frac{\Delta \lambda_{h}}{\bar{\lambda}_{h}} \frac{\Delta \lambda_{k}}{\bar{\lambda}_{k}}, & h, k=1, \ldots, \mu \\
\tilde{\lambda}_{3}:=\frac{\Delta \lambda_{h}}{\bar{\lambda}_{h}} \frac{\Delta \lambda_{k}}{\bar{\lambda}_{k}} \frac{\Delta \lambda_{j}}{\bar{\lambda}_{j}}, & h, k, j=1, \ldots, \mu \\
\vdots & \end{cases}
\end{aligned}
$$


Given that the accuracy requirement of the application allows the truncation of Taylor series expansion in (4.6) by selecting the first $p$ terms and considering (4.7) and (4.8), an approximate representation for the multi-parameter circuit equations in (4.3) is obtained as

$$
\begin{aligned}
\left(\tilde{\mathbf{E}}_{0}+\tilde{\mathbf{E}}_{1} \tilde{\lambda}_{1}+\cdots+\tilde{\mathbf{E}}_{p} \tilde{\lambda}_{p}\right) \mathbf{X}(\tilde{\boldsymbol{\lambda}}) & =\mathbf{B U}(s) \\
\mathbf{Y}(\tilde{\boldsymbol{\lambda}}) & =\mathbf{L X}(\tilde{\boldsymbol{\lambda}})
\end{aligned}
$$

where

$$
\tilde{\boldsymbol{\lambda}}=\left[\tilde{\lambda}_{1}, \ldots, \tilde{\lambda}_{p}\right]^{\top}
$$

such that the defined parameters $\tilde{\lambda}_{i}, i=1, \ldots, p$ are some functions of the original parameters $\lambda_{i}, i=1, \ldots, \mu$ in (4.3). This is an approximation for the original formulation for the parametrized circuit in (4.3) based on the Taylor series approximation.

\subsubsection{Multi-parameter Moments of Parametric Circuits}

Given $\tilde{\mathbf{E}}_{0}$ in (4.7), the circuit's matrix pencil evaluated at the parameters' nominal values, is invertible, from $(4.9 \mathrm{a})$, we get

$$
\left(\mathbf{I}_{n}-\mathbf{M}_{1} \tilde{\lambda}_{1}-\cdots-\mathbf{M}_{p} \tilde{\lambda}_{p}\right) \mathbf{X}(\boldsymbol{\lambda})=\mathbf{B}_{M} \mathbf{U}(s)
$$

where

$$
\begin{aligned}
\mathbf{M}_{i} & =-\tilde{\mathbf{E}}_{0}^{-1} \tilde{\mathbf{E}}_{i}, \quad i=1,2, \ldots, p \\
\mathbf{B}_{M} & =\tilde{\mathbf{E}}_{0}^{-1} \mathbf{B}
\end{aligned}
$$

and $\mathbf{I}_{n}$ is an $n \times n$ identity matrix. 
Solving (4.11) for the circuit variables $\mathbf{X}(\boldsymbol{\lambda})$ leads to

$$
\begin{gathered}
\mathbf{X}(\tilde{\boldsymbol{\lambda}})=\left[\mathbf{I}_{n}-\left(\mathbf{M}_{1} \tilde{\lambda}_{1}+\cdots+\mathbf{M}_{p} \tilde{\lambda}_{p}\right)\right]^{-1} \mathbf{B}_{M} \mathbf{U}(s) \\
=\sum_{m=0}^{\infty}\left(\mathbf{M}_{1} \tilde{\lambda}_{1}+\cdots+\mathbf{M}_{p} \tilde{\lambda}_{p}\right)^{m} \mathbf{B}_{M} \mathbf{U}(s) \\
=\sum_{m=0}^{\infty} \sum_{k_{2}=0}^{m-\left(k_{3}+\cdots+k_{p}\right)} \cdots \sum_{k_{p-1}=0}^{m-k_{p}} \sum_{k_{p}=0}^{m}\left[\mathbf{F}_{k_{2}, \ldots, k_{p}}^{m}\left(\mathbf{M}_{1}, \ldots, \mathbf{M}_{p}\right) \mathbf{B}_{M}\right] \tilde{\lambda}_{1}^{m-\left(k_{2}, \ldots, k_{p}\right)} \tilde{\lambda}_{2}^{k_{2}} \ldots \tilde{\lambda}_{p}^{k_{p}} \mathbf{U}(s)
\end{gathered}
$$

where

$$
\mathbf{F}_{k_{2}, \ldots, k_{p}}^{m}\left(\mathbf{M}_{1}, \ldots, \mathbf{M}_{p}\right)= \begin{cases}0, & \text { if } k_{i} \notin\{0,1, \ldots, m\}, \quad i=2, \ldots, p \\ 0, & \text { if } k_{2}+\ldots+k_{p} \notin\{0,1, \ldots, m\} \\ \mathbf{I}_{n} \quad & \text { if } m=0 \\ \mathbf{M}_{1} \mathbf{F}_{k_{2}, \ldots, k_{p}}^{m-1}\left(\mathbf{M}_{1}, \ldots, \mathbf{M}_{p}\right)+\mathbf{M}_{2} \mathbf{F}_{k_{2}-1, \ldots, k_{p}}^{m-1}\left(\mathbf{M}_{1}, \ldots,\right. \\ \left.\mathbf{M}_{p}\right)+\ldots+\mathbf{M}_{p} \mathbf{F}_{k_{2}, \ldots, k_{p}-1}^{m-1}\left(\mathbf{M}_{1}, \ldots, \mathbf{M}_{p}\right) \\ \vdots\end{cases}
$$

The coefficients in the series expansion of the circuit variables in (4.13) are referred to as the "circuit moments".

$$
\mathbf{N}_{m} \triangleq \sum_{k_{2}=0}^{m-\left(k_{3}+\cdots+k_{p}\right)} \cdots \sum_{k_{p-1}=0}^{m-k_{p}} \sum_{k_{p}=0}^{m} \mathbf{F}_{k_{2}, \ldots, k_{p}}^{m}\left(\mathbf{M}_{1}, \ldots, \mathbf{M}_{p}\right) \mathbf{B}_{M}
$$

For example, a few leading circuit moments are presented in Table 4.1. Defining the parametric transfer function of the circuit as a multi-variable function relating inputs to the outputs, from (4.13) and (4.3b) we get 


$$
\begin{aligned}
& \mathbf{H}(\tilde{\boldsymbol{\lambda}})= \\
& \quad \mathbf{L} \sum_{m=0}^{\infty} \sum_{k_{2}=0}^{m-\left(k_{3}+\cdots+k_{p}\right)} \ldots \sum_{k_{p-1}=0}^{m-k_{p}} \sum_{k_{p}=0}^{m}\left[\mathbf{F}_{k_{2}, \ldots, k_{p}}^{m}\left(\mathbf{M}_{1}, \ldots, \mathbf{M}_{p}\right) \mathbf{B}_{M}\right] \tilde{\lambda}_{1}^{m-\left(k_{2}, \ldots, k_{p}\right)} \tilde{\lambda}_{2}^{k_{2}} \ldots \tilde{\lambda}_{p}^{k_{p}}
\end{aligned}
$$

Hence, the corresponding moments of the transfer function are

$$
\breve{\mathbf{N}}_{m}=\mathbf{L N}_{m}
$$

\begin{tabular}{|c|c|c|}
\hline $\begin{array}{c}\text { Moments } \\
\text { Order }\end{array}$ & $\begin{array}{l}\text { Moments } \\
\qquad \mathbf{N}_{m}\end{array}$ & $\begin{array}{c}\text { Coefficients } \\
\text { of }\end{array}$ \\
\hline 0 -th & $\mathbf{B}_{M}$ & - \\
\hline $1-\mathrm{st}$ & $\begin{array}{c}\mathbf{M}_{i} \mathbf{B}_{M} \\
i=1,2, \ldots, p\end{array}$ & $\begin{array}{c}\tilde{\lambda}_{i} \\
i=1,2, \ldots, p\end{array}$ \\
\hline 2-nd & $\begin{array}{c}\mathbf{M}_{i}^{2} \mathbf{B}_{M} \\
i=1,2, \ldots, p \\
\left(\mathbf{M}_{1} \mathbf{M}_{i}+\mathbf{M}_{i} \mathbf{M}_{1}\right) \mathbf{B}_{M} \\
i=2, \ldots, p \\
\left(\mathbf{M}_{2} \mathbf{M}_{i}+\mathbf{M}_{i} \mathbf{M}_{2}\right) \mathbf{B}_{M} \\
i=3, \ldots, p \\
\vdots \\
\left(\mathbf{M}_{p-1} \mathbf{M}_{p}+\mathbf{M}_{p} \mathbf{M}_{p-1}\right) \mathbf{B}_{M}\end{array}$ & $\begin{array}{c}\tilde{\lambda}_{i} \\
i=1,2, \ldots, p \\
\tilde{\lambda}_{1} \tilde{\lambda}_{i} \\
i=2, \ldots, p \\
\tilde{\lambda}_{2} \tilde{\lambda}_{i} \\
i=3, \ldots, p \\
\vdots \\
\tilde{\lambda}_{p-1} \tilde{\lambda}_{p}\end{array}$ \\
\hline : & . & $\vdots$ \\
\hline
\end{tabular}

Table 4.1: Circuit moments. 


\subsection{Reduction Scheme for Parametric Circuits}

Following the Galerkin-projection scheme presented in Chapter 3, the reduced-order model for the parametric circuit in (4.9) can be obtained using the variable change as

$$
\mathbf{Z}(\tilde{\boldsymbol{\lambda}}) \triangleq \mathbf{V}^{\top} \mathbf{X}(\tilde{\boldsymbol{\lambda}})
$$

and through congruent transformation. It is

$$
\begin{aligned}
\left(\hat{\mathbf{E}}_{0}+\hat{\mathbf{E}}_{1} \tilde{\lambda}_{1}+\cdots+\hat{\mathbf{E}}_{p} \tilde{\lambda}_{p}\right) \mathbf{Z}(\tilde{\boldsymbol{\lambda}}) & =\hat{\mathbf{B}} \mathbf{U}(s) \\
\mathbf{Y}(\tilde{\boldsymbol{\lambda}}) & =\hat{\mathbf{L}} \mathbf{Z}(\tilde{\boldsymbol{\lambda}})
\end{aligned}
$$

where

$$
\begin{array}{rccc}
\hat{\mathbf{E}}_{0} \triangleq \mathbf{V}^{\top} \tilde{\mathbf{E}}_{0} \mathbf{V} & \hat{\mathbf{E}}_{1} \triangleq \mathbf{V}^{\top} \tilde{\mathbf{E}}_{1} \mathbf{V} & \ldots & \hat{\mathbf{E}}_{p} \triangleq \mathbf{V}^{\top} \tilde{\mathbf{E}}_{p} \mathbf{V} \\
& \hat{\mathbf{B}} \triangleq \mathbf{V}^{\top} \mathbf{B} & \hat{\mathbf{L}} \triangleq \mathbf{L V} &
\end{array}
$$

and $\mathbf{V} \in \mathbb{R}^{n \times q}$ is the order-reduction projection matrix.

\subsection{Construction of Projection Matrix}

Using the projection framework described above as an effective reduction strategy has been extensively explored in literature for parametric linear circuits $[45,50,52-$ 56]. The key step in any projection-based reduction is constructing the reduction projection matrix $\mathbf{V}$ used in equation (4.19). The method employed to obtain $\mathbf{V}$ is the main differentiating factor between the reduction methods. Finding a suitable projection matrix is the main task in any MOR method. A common practice in forming $\mathbf{V}$ is established based on the moment-matching idea. It is to ensure that 
the response of the reduced-order model $\mathbf{Y}(\boldsymbol{\lambda})$ in (4.19) will share a certain number of the leading frequency and parameter moments of the responses from the original circuit response in (4.14).

The following sections will elaborate on the construction of the projection operator using the "moments of the circuit" as related to the PMOR application.

\subsubsection{Explicit Moment-Matching Based Approach}

In explicit moment-matching based approach discussed in [54], forming the reducedorder system in (4.19) is primarily carried out by constructing an orthogonal basis set $\mathbf{V} \in \mathbb{R}^{n \times q}$ whose column vectors form a basis for the Krylov subspace spanned by the moments of the circuit with respect to the parameters (4.15).

$$
\begin{aligned}
\operatorname{colsp}(\mathbf{V}) & =\operatorname{span}\left\{\mathbf{B}_{M}, \mathbf{M}_{1} \mathbf{B}_{M}, \ldots, \mathbf{M}_{p} \mathbf{B}_{M}, \mathbf{M}_{1}^{2} \mathbf{B}_{M},\left(\mathbf{M}_{1} \mathbf{M}_{2}+\mathbf{M}_{2} \mathbf{M}_{1}\right) \mathbf{B}_{M}, \ldots\right. \\
& \left.\ldots,\left(\mathbf{M}_{1} \mathbf{M}_{p}+\mathbf{M}_{p} \mathbf{M}_{1}\right) \mathbf{B}_{M}, \mathbf{M}_{2}^{2} \mathbf{B}_{M},\left(\mathbf{M}_{2} \mathbf{M}_{3}+\mathbf{M}_{3} \mathbf{M}_{2}\right) \mathbf{B}_{M}, \ldots\right\} \\
& =\operatorname{span}\left\{\bigcup_{m=0}^{m_{q}} \bigcup_{k_{2}=0}^{m-\left(k_{p}+\cdots+k_{3}\right)} \ldots \bigcup_{k_{p-1}=0}^{m-k_{p}} \bigcup_{k_{p}=0}^{m} \mathbf{F}_{k_{2}, \ldots, k_{p}}^{m}\left(\mathbf{M}_{1}, \ldots, \mathbf{M}_{p}\right) \mathbf{B}_{M}\right\}
\end{aligned}
$$

By using a suitable orthogonalization algorithm such as the Gram-Schmidt process (i.e., "qr ()" in MATLAB $\left.{ }^{\circledR}\right)$ a set of $q$ basis is computed to form the orthonormal projection matrix $\mathbf{V}$ ensuring

$$
\mathbf{V}^{\top} \mathbf{V}=\mathbf{I}_{q}
$$

where $\mathbf{I}_{q}$ is a $q \times q$ identity matrix. From (4.20), one should also note that the order of the reduced model is proportional to the number of inputs to the circuit $\left(q \propto n_{\text {in }}\right)$. Its impairing effect on the efficiency of the reduced-order models for multi-input circuits will be dealt with in the following sections. 


\section{Multi-parameter Moments of Parametric Reduced-Order Model}

Similar to the steps shown in Section 4.1.1, the moments of the parametric reducedorder model can be obtained.

$$
\hat{\mathbf{N}}_{m} \triangleq \sum_{k_{2}=0}^{m-\left(k_{3}+\cdots+k_{p}\right)} \cdots \sum_{k_{p-1}=0}^{m-k_{p}} \sum_{k_{p}=0}^{m} \hat{\mathbf{F}}_{k_{2}, \ldots, k_{p}}^{m}\left(\hat{\mathbf{M}}_{1}, \ldots, \hat{\mathbf{M}}_{p}\right) \hat{\mathbf{B}}_{M}
$$

where

$$
\begin{aligned}
\hat{\mathbf{M}}_{i} & =-\hat{\mathbf{E}}_{0}^{-1} \hat{\mathbf{E}}_{i}, \quad i=1,2, \ldots, p \\
\hat{\mathbf{B}}_{M} & =\hat{\mathbf{E}}_{0}^{-1} \hat{\mathbf{B}}
\end{aligned}
$$

and $\hat{\mathbf{E}}_{i}$ for $i=0, \ldots, p$ are given in $(4.19 \mathrm{c})$.

\section{PMOR Moment-Matching Property}

Theorem 2 (Multi-moment matching). The first q leading moments of the transfer function of the parametric reduced-order model in (4.19) generated using the projection matrix $\mathbf{V}$ in (4.20) match those of the original (unreduced) circuit in (4.9)

$$
\begin{array}{r}
\hat{\mathbf{F}}_{k_{2}, \ldots, k_{p}}^{m}\left[-\left(\mathbf{V}^{T} \tilde{\mathbf{E}}_{0} \mathbf{V}\right)^{-1} \mathbf{V}^{T} \tilde{\mathbf{E}}_{1} \mathbf{V}, \ldots,\left[-\left(\mathbf{V}^{T} \tilde{\mathbf{E}}_{0} \mathbf{V}\right)^{-1} \mathbf{V}^{T} \tilde{\mathbf{E}}_{p} \mathbf{V}\right]\left[-\left(\mathbf{V}^{T} \tilde{\mathbf{E}}_{0} \mathbf{V}\right)^{-1} \mathbf{V}^{T} \mathbf{B}=\right.\right. \\
\mathbf{V}^{T} \mathbf{F}_{k_{2}, \ldots, k_{p}}^{m}\left[-\tilde{\mathbf{E}}_{0}^{-1} \tilde{\mathbf{E}}_{1}, \ldots,-\tilde{\mathbf{E}}_{0}^{-1} \tilde{\mathbf{E}}_{p}\right]-\tilde{\mathbf{E}}_{0}^{-1} \mathbf{B}
\end{array}
$$

To prove that the parameterized model order reduction matches the original moments in Theorem 2, first the following lemmas should be considered.

Lemma 1. If projection matrix $\mathbf{V}$ is an orthonormal matrix $\mathbf{V} \in \mathbb{R}^{n \times q}, \mathbf{V}^{T} \mathbf{V}=\mathbf{I}_{q} \in$ $\mathbb{R}^{q \times q}$ and $\mathbf{w}$ is any random vector in the column span of the matrix $\mathbf{V}, \mathbf{w} \in \operatorname{colsp}(\mathbf{V})$, then $\mathbf{V} \mathbf{V}^{T} \mathbf{w}=\mathbf{w}$. 
Proof. Taking the reference from [43], if $\mathbf{w} \in \operatorname{colsp}(\mathbf{V})$ then there must be a vector $\mathbf{y}$ such that $\mathbf{V y}=\mathbf{w}$. Substituting $\mathbf{V} \mathbf{V}^{T} \mathbf{w}=\mathbf{V V}^{T} \mathbf{V} \mathbf{y}=\mathbf{V} \mathbf{y}=\mathbf{w}$.

Lemma 2. If projection matrix $\mathbf{V}$ is an orthonormal matrix $\mathbf{V} \in \mathbb{R}^{n \times q}, \mathbf{V}^{T} \mathbf{V}=$ $\mathbf{I}_{q} \in \mathbb{R}^{q \times q}$ and $\mathbf{w}$ is a vector such that $\tilde{\mathbf{E}}_{0}^{-1} \mathbf{w} \in \operatorname{colsp}(\mathbf{V})$, then $\left(\mathbf{V}^{T} \tilde{\mathbf{E}}_{0} \mathbf{V}\right)^{-1} \mathbf{V}^{T} \mathbf{w}=$ $\mathbf{V}^{T} \tilde{\mathbf{E}}_{0}^{-1} \mathbf{w}$.

Proof. As from [8], to prove that $\mathbf{y}=\mathbf{V}^{T} \tilde{\mathbf{E}}_{0}^{-1} \mathbf{w}$ is a solution for the linear system $\left(\mathbf{V}^{T} \tilde{\mathbf{E}}_{0} \mathbf{V}\right) \mathbf{y}=\mathbf{V}^{T} \mathbf{w}$. Substituting, $\left(\mathbf{V}^{T} \tilde{\mathbf{E}}_{0} \mathbf{V}\right) \mathbf{y}=\left(\mathbf{V}^{T} \tilde{\mathbf{E}}_{0} \mathbf{V}\right) \mathbf{V}^{T} \tilde{\mathbf{E}}_{0}^{-1} \mathbf{w}$. Since $\tilde{\mathbf{E}}_{0}^{-1} \mathbf{w} \in$ $\operatorname{colsp}(\mathbf{V})$ from Lemma 1, that means $\mathbf{V}^{T} \tilde{\mathbf{E}}_{0} \mathbf{V} \mathbf{V}^{T} \tilde{\mathbf{E}}_{0}^{-1} \mathbf{w}=\mathbf{V}^{T} \tilde{\mathbf{E}}_{0} \tilde{\mathbf{E}}_{0}^{-1} \mathbf{w}=\mathbf{V}^{T} \mathbf{w}$.

Lemma 3. If $\mathbf{F}_{k_{2}, \ldots, k_{p}}^{m}\left(\mathbf{M}_{1}, \ldots, \mathbf{M}_{p}\right)$ is a matrix form using equation (4.14), and $\mathbf{V} \in \mathbb{R}^{n \times q}$ is an orthonormal matrix constructed such that equation (4.21) holds, then (4.24) holds for $m=0,1, \ldots, m_{1}$.

The proofs for Lemma-3 and Theorem-2 are given in Appendices A and B, respectively.

The reduced model is dependent on the projection matrix $\mathbf{V}$. To find the columns of $\mathbf{V}$, we used the explicit method shown in equation (4.20). However, this also suffers from the same problems discussed in section 3.1. The sequence in (4.20) converges to higher-order terms. In the next section, an implicit moment matching technique is discussed.

\subsubsection{Implicit Moment-Matching Based Approach}

This section presents a stable algorithm based on implicit moment matching to resolve the numerical instability problem in explicit moment matching [53]. This algorithm can be suitable for both Multiple-Input Multiple-Output (MIMO) and Single-Input Single-Output (SISO). 
From the parametric state-space equation as given in (4.13), we can get

$$
\begin{aligned}
\mathbf{X}(\tilde{\boldsymbol{\lambda}})= & \sum_{m=0}^{\infty}\left[\tilde{\lambda}_{1} \mathbf{M}_{1}+\cdots+\tilde{\lambda}_{p} \mathbf{M}_{p}\right]^{m} \mathbf{B}_{M} \mathbf{U}(s) \\
= & \mathbf{B}_{M} \mathbf{U}(s)+\left[\tilde{\lambda}_{1} \mathbf{M}_{1}+\cdots+\tilde{\lambda}_{p} \mathbf{M}_{p}\right] \mathbf{B}_{M} \mathbf{U}(s) \\
& +\left[\tilde{\lambda}_{1} \mathbf{M}_{1}+\cdots+\tilde{\lambda}_{p} \mathbf{M}_{p}\right]^{2} \mathbf{B}_{M} \mathbf{U}(s)+\ldots \\
& +\left[\tilde{\lambda}_{1} \mathbf{M}_{1}+\cdots+\tilde{\lambda}_{p} \mathbf{M}_{p}\right]^{j} \mathbf{B}_{M} \mathbf{U}(s)+\ldots
\end{aligned}
$$

where

$$
\begin{aligned}
\mathbf{M}_{i} & =-\tilde{\mathbf{E}}_{0}^{-1} \tilde{\mathbf{E}}_{i} \quad i=1,2, \ldots, p, \\
\mathbf{B}_{M} & =-\tilde{\mathbf{E}}_{0}^{-1} \mathbf{B} .
\end{aligned}
$$

By defining

$$
\begin{aligned}
& \mathbf{x}_{0}=\mathbf{B}_{M}, \\
& \mathbf{x}_{1}=\left[\tilde{\lambda}_{1} \mathbf{M}_{1}+\cdots+\tilde{\lambda}_{p} \mathbf{M}_{p}\right] \mathbf{B}_{M}, \\
& \mathbf{x}_{2}=\left[\tilde{\lambda}_{1} \mathbf{M}_{1}+\cdots+\tilde{\lambda}_{p} \mathbf{M}_{p}\right]^{2} \mathbf{B}_{M}, \\
& \mathbf{x}_{j}=\left[\tilde{\lambda}_{1} \mathbf{M}_{1}+\cdots+\tilde{\lambda}_{p} \mathbf{M}_{p}\right]^{j} \mathbf{B}_{M},
\end{aligned}
$$


the following recursive relations between the new variables in (4.27) is realized,

$$
\begin{aligned}
& \mathbf{x}_{0}=\mathbf{B}_{M}, \\
& \mathbf{x}_{1}=\left[\tilde{\lambda}_{1} \mathbf{M}_{1}+\cdots+\tilde{\lambda}_{p} \mathbf{M}_{p}\right] \mathbf{x}_{0}, \\
& \mathbf{x}_{2}=\left[\tilde{\lambda}_{1} \mathbf{M}_{1}+\cdots+\tilde{\lambda}_{p} \mathbf{M}_{p}\right] \mathbf{x}_{1}, \\
& \mathbf{x}_{j}=\left[\tilde{\lambda}_{1} \mathbf{M}_{1}+\cdots+\tilde{\lambda}_{p} \mathbf{M}_{p}\right] \mathbf{x}_{j-1},
\end{aligned}
$$

Considering the coefficient of $\mathbf{x}_{j}, j=0,1, \ldots$ in (4.28), we have

$$
\begin{aligned}
& \mathbf{R}_{0}=\mathbf{B}_{M} \\
& \mathbf{R}_{1}=\left[\tilde{\lambda}_{1} \mathbf{R}_{0}+\cdots+\tilde{\lambda}_{p} \mathbf{R}_{0}\right], \\
& \mathbf{R}_{2}=\left[\tilde{\lambda}_{1} \mathbf{R}_{1}+\cdots+\tilde{\lambda}_{p} \mathbf{R}_{1}\right], \\
& \mathbf{R}_{j}=\left[\tilde{\lambda}_{1} \mathbf{R}_{j-1}+\cdots+\tilde{\lambda}_{p} \mathbf{R}_{j-1}\right]
\end{aligned}
$$

The next step is to use the subspace defined by the moments in (4.29) to construct the orthogonal projection matrix $\mathbf{V}$ spanning the subspace of the moments as

$$
\operatorname{colsp}(\mathbf{V})=\operatorname{span}\left\{\mathbf{R}_{0}, \mathbf{R}_{1}, \mathbf{R}_{2}, \ldots, \mathbf{R}_{m}\right\}
$$

The numerically stable algorithm to compute the $\mathbf{V}$ is discussed in Algorithm 2. All the vectors of $\mathbf{V}$ are orthogonalized with the help of Modified Gram-Schmidt (MGS). Also, there is no limitation on the number of parameters and most important the computational cost of $\tilde{\mathbf{E}}^{-1} \tilde{\mathbf{E}}_{j}$ grows linearly as the number of parameter increase. However, the cost of the orthogonalization step grows quadratically with parameters.

Let's discuss some important points of Algorithm 2: 


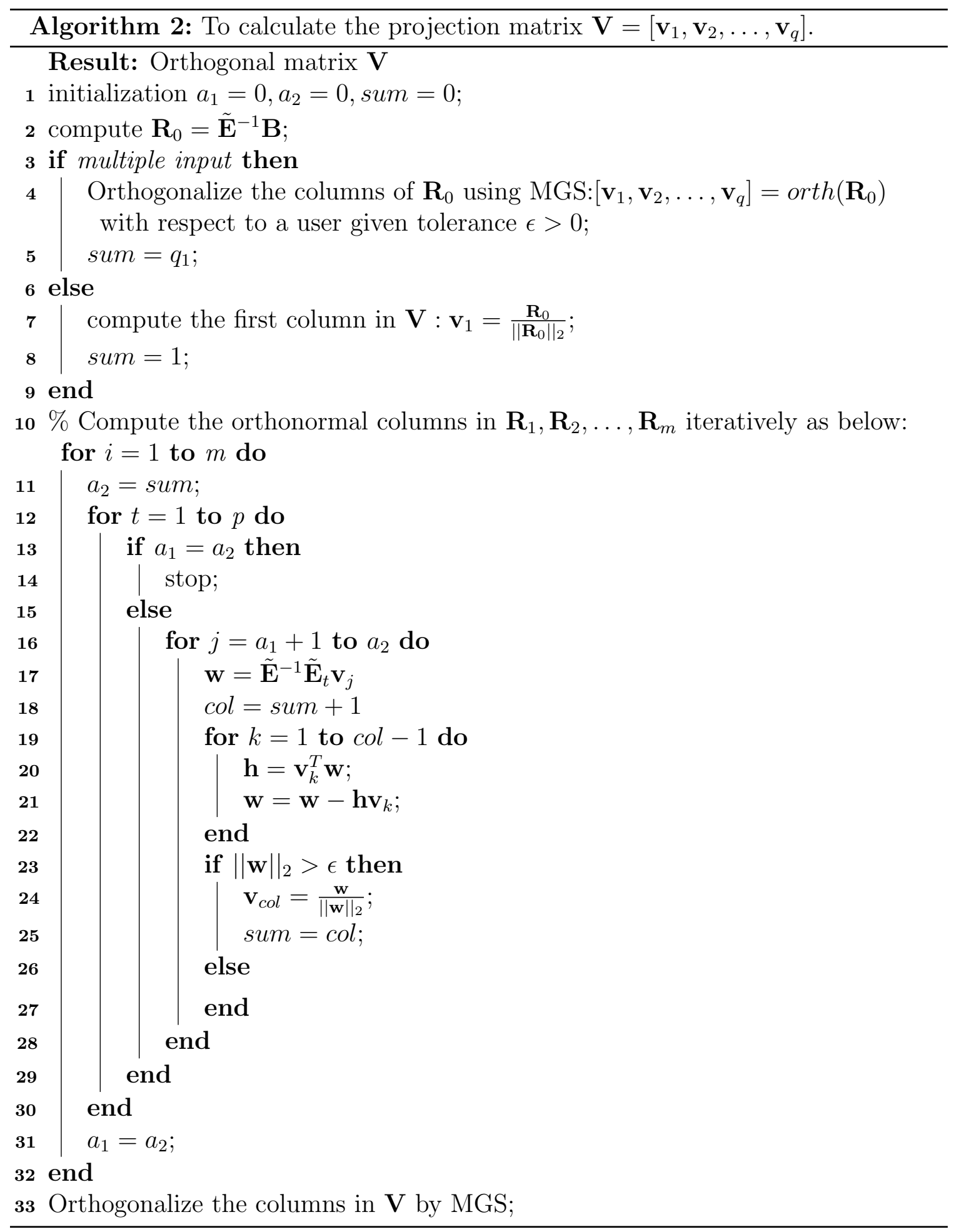


1. The term $\tilde{\mathbf{E}}^{-1}$ (sparse) used in step 2 and 17 can be computed once before starting the algorithm by matrix factorization (LU decomposition) and when ever needed a forward/backward method to solve for that term. Therefore, only one-time matrix factorization is required in the whole algorithm making the algorithm faster.

2. To find the $\tilde{\mathbf{E}}_{t} \mathbf{v}_{j}$ in step 17 required matrix-vector multiplication. Then used the factors of $\tilde{\mathbf{E}}$ to solve for $\mathbf{w}$.

3. When used for multiple inputs, $\mathbf{B}$ is matrix. Orthogonalization of $\mathbf{R}_{0}=\tilde{\mathbf{E}}^{-1} \mathbf{B}$ is needed before use to find the next $\mathbf{R}_{i}$. To monitor the columns of $\mathbf{V}$ a sum variable is initialized.

4. In first for loop $\mathrm{m}$ represents the highest order of moment that is going to be computed which is given by the user.

5. The $\mathrm{t}$ in step 12 refer to how many parameters $\tilde{\lambda}$ are going to be used corresponding to the coefficient $\tilde{\mathbf{E}}^{-1} \mathbf{E}_{t}$.

6. $a_{2}-a_{1}$ is the number of columns adding to $\mathbf{V}$ during $\mathbf{R}_{i-1}$-th calculation.

7. When $a_{2}=a_{1}$, all the columns calculated in $\mathbf{v}_{\text {col }}$ are deflated because they are linearly dependent on the previous columns in that matrix. This implies that there is no vector pending for $\mathbf{R}_{i-1}$. Hence, the algorithm has to stops.

8. In step 16 , $\mathrm{j}$ represents the $\mathrm{j}$-th column in $\mathbf{V}$, which corresponds to a vector in $\mathbf{R}_{i-1}$.

9. Steps 19 to 25 are the process of MGS. All the columns in $\mathbf{V}$ are orthogonalized with the previous columns in $\mathbf{V}$ by MGS. The subscript col represents the current column $\mathbf{v}_{c o l}$ in $\mathbf{V}$. 
10. The equation used in step $23,\|\mathbf{w}\|_{2}>\epsilon$ is the criterion for the deflate vectors in $\mathbf{R}_{i}$ that are linearly dependent on the previous vectors in $\mathbf{V}$. That does not mean all the columns in $\mathbf{R}_{i}$ are going to be linearly dependent on the previous vectors in $\mathbf{V}$.

11. In the last step $33, \mathbf{V}$ is orthonormalized again using MGS to minimize $\left\|\mathbf{V}^{T} \mathbf{V}-\mathbf{I}\right\|_{2}$. The final projection matrix $\mathbf{V}$ has q columns.

12. When there is only one parameter (frequency/time), in the second for loop $p=1$ and the algorithm shrinks to a block Arnoldi form.

However, this method also suffers the same problems that we have seen with the Arnoldi algorithms. When a system is large enough with more parameters to work with, the reduced parametric system will also be large and dense that requires more computational cost than the original system.

\subsection{Order Growth and Computational Complexity}

A theoretical measure for the order of parametrized reduced model $q$, which is also the number of columns in the projection matrix $\mathbf{V}$, is given by

$$
q=\sum_{k=0}^{p_{\mu}} \frac{(k+\mu-1) !}{k !(\mu-1) !}
$$

where $\mu$ is the number of parameters and $p_{\mu}=\max \left(p_{1}, \ldots, p_{\mu}\right)$ is the largest order of the parameter derivatives to match $[29,54]$. One way to improve accuracy is to increase $p_{\mu}$. For a successful reduction, $p_{\mu}$ should be selected large enough to ensure sufficient accuracy. For a given $p_{\mu}$, it is straightforward to see from (4.31) that as the number of the parameters increases, the size of paramedic reduced-order models and hence, the associated computational complexity increases. 


\subsection{Summary and Discussions}

This chapter outlined MOR concepts and their application in the context of Parameterized MOR (PMOR). The main objective of PMOR is to create a reduced system of equations whose solution tracks the variations of the circuit response with respect to the variation in some design parameters. The under-laying idea in the presented approach to reduce parametrized circuits relies on extending the notion of moment-matching used for linear MOR. The procedural steps were presented based on explicit and implicit moment-matching schemes. Orthogonalizing the explicitly computed moments to form the reduction-projection matrix succeeds in creating a parametrized reduced-order model for circuits with very few parameters. However, a generalization of the idea for multi-parameter circuits exposes high numerical instability. On the contrary, an algorithm based on implicit moment matching is numerically stable and theoretically can handle any number of parameters and any number of moments to match.

Moreover, the size of paramedic reduced-order models increases with the number of inputs to the circuit. This fact would spawn new challenges to parameterized modelorder reduction. In general, the problem is arduously difficult for multi-parameter circuits with many ports. The next chapter will address this issue. 


\section{Chapter 5}

\section{Reduction of Massively Coupled Interconnect Structures: Clustering Scheme}

When working with high frequencies, it is crucial to model interconnects accurately. Using distributed Multi-conductor Transmission Lines (MTLs) [60] models can serve the purpose of accuracy; however, they are CPU expensive to simulate for mixed time/frequency problems. There are some methods proposed in the literature, e.g., based on macromodeling [61-65] and waveform relaxation [66-68] to alleviate the simulation cost. The resulting models are dependent on the terminations (at the input/output terminals) and input stimuli of the circuit. Any changes in stimuli or terminations require altering the model and hence, repeating the simulation.

The macromodeling based on discretization is an alternative approach that is not dependent on the input signals or the port terminations. One also can readily incorporate the (netlist of) resulting circuit models in the commercial circuit simulators for system-level analysis. For most applications where the accuracy requirement permits, circuit-wise modeling based on segmentation has become the method of choice. The resulting circuit MNA equations typically become extensively large, especially 
when working with large bandwidth. This becomes even worse when dealing with the coupled multi-transmission lines.

Model-Order Reduction is a natural solution to reduce the complexity of such large models while ensuring adequate accuracy besides preserving important physical characteristics. Several numerically stable techniques based on implicit moment matching and congruence transformation are in the literature $[41,48,69-74]$ to serve this purpose.

This section explains an algorithm for efficiently reducing massively coupled interconnect structures modeled as large networks with many terminals.

\subsection{MOR for Multi-Input Networks}

Using the moment-matching projection-based MOR methods to reduce a circuit with $n_{\text {in }}$ inputs while matching $m$ block moments, the order of the reduced model is $q=m \times n_{i n}$. This emphasizes that applying MOR to reduce circuits with a small number of ports $\left(n_{i n}\right)$ can lead to reduced models much smaller than the original ones. However, to match the same $m$ block-moments, the order of reduced system grows proportionally with the increase in the number of ports. Additionally, the non-zero entries in the reduced models also increase rapidly with the number of the ports [75]. This implies that the reduced system becomes denser and more CPU expansive. For the circuits with many inputs such as coupled MTL, this can lead to noticeable efficiency degradation, undermining the advantages expected from MOR [13].

For the efficient reduction of linear circuits with many terminals, an algorithm has been developed relying on the superposition theorem $[6,76]$. The superposition paradigm as a fundamental rule in the analysis of multi-input circuits allows dealing with one (or more) inputs at a time. The overall response is obtained by superposing the responses to the excitation at individual inputs, as illustrated in Figure 5.1. This 


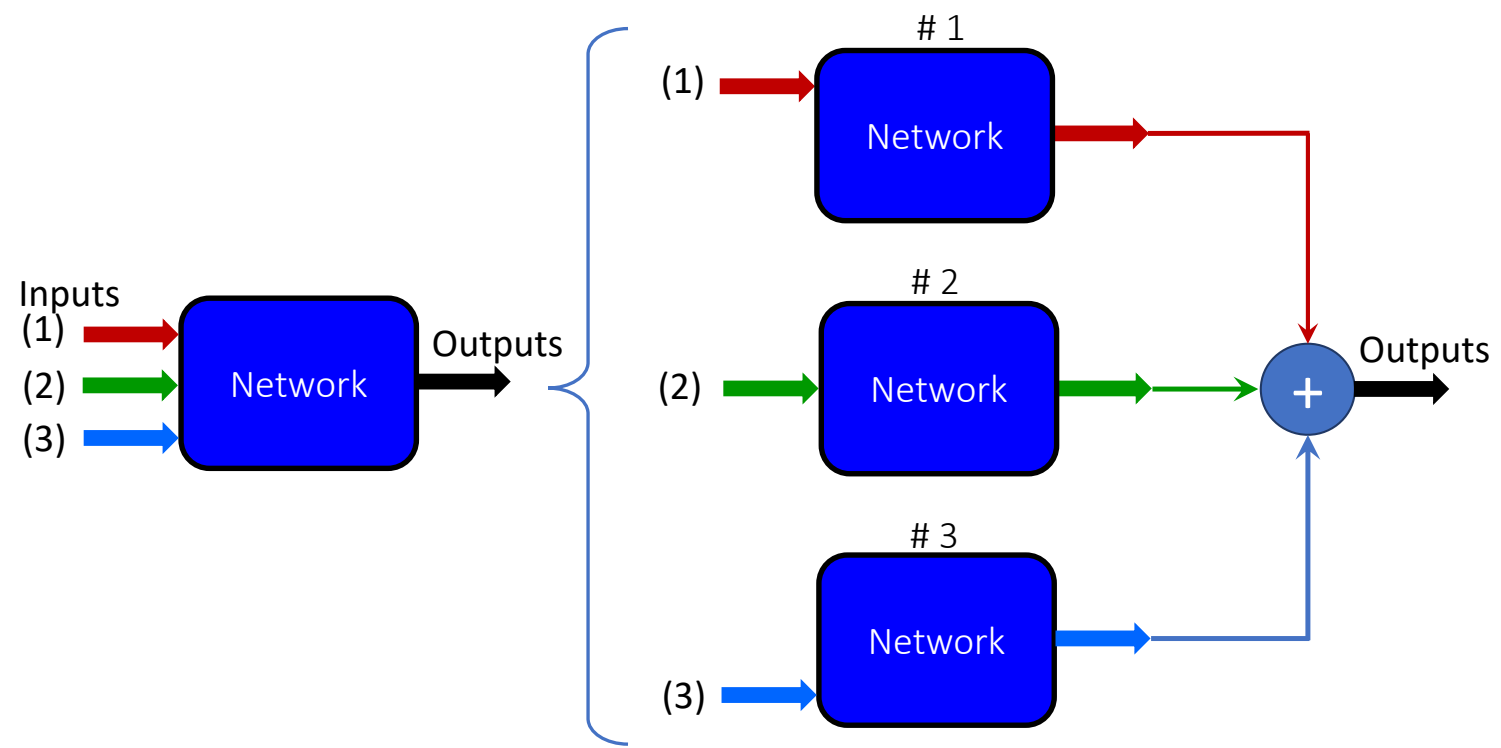

Figure 5.1: Overall response is obtained by superposing the responses caused by excitation at each input.

paradigm is used to devise a macromodeling approach by considering a "cluster" of the inputs at each time. Then the overall responses are constructing by superposing the outputs for the inputs associated with clusters.

\subsection{MOR for MTL}

By pushing the idea presented in Section 5.1 to the case of massively coupled interconnect structures, a reduction technique is developed that uses the flexible clustering of the transmission lines of the original system to form the individual subsystems. Each subsystem contains all the lines where only a subset of them is considered active. Then these subsystems are merged based on the superposition theorem to find the overall reduced system.

The most significant advantage of this algorithm for model order reduction of interconnect structures is that the resulting reduced model is sparse and block-diagonal. The approach, based on a fundamental paradigm in circuit theory, does not result 
in diminishing accuracy. Moreover, there is no assumption made for the correlation between the responses at ports. All the low-rank approximation-based terminal reduction methods [77-81] developed based on the exploitation of such correlation generally suffer the accuracy degradation. Understandably, any correlation between the port responses depends closely on the input waveform and frequency. However, the method explained here by not relying on correlation assumption dismisses any related accuracy degradation.

\subsection{Reduction via Clustering}

This section focuses on the clustering-based reduction algorithm for the multi-port large order linear systems with massively coupled transmission line structures. Consider the $N$ conductor interconnect shown in 5.2 .

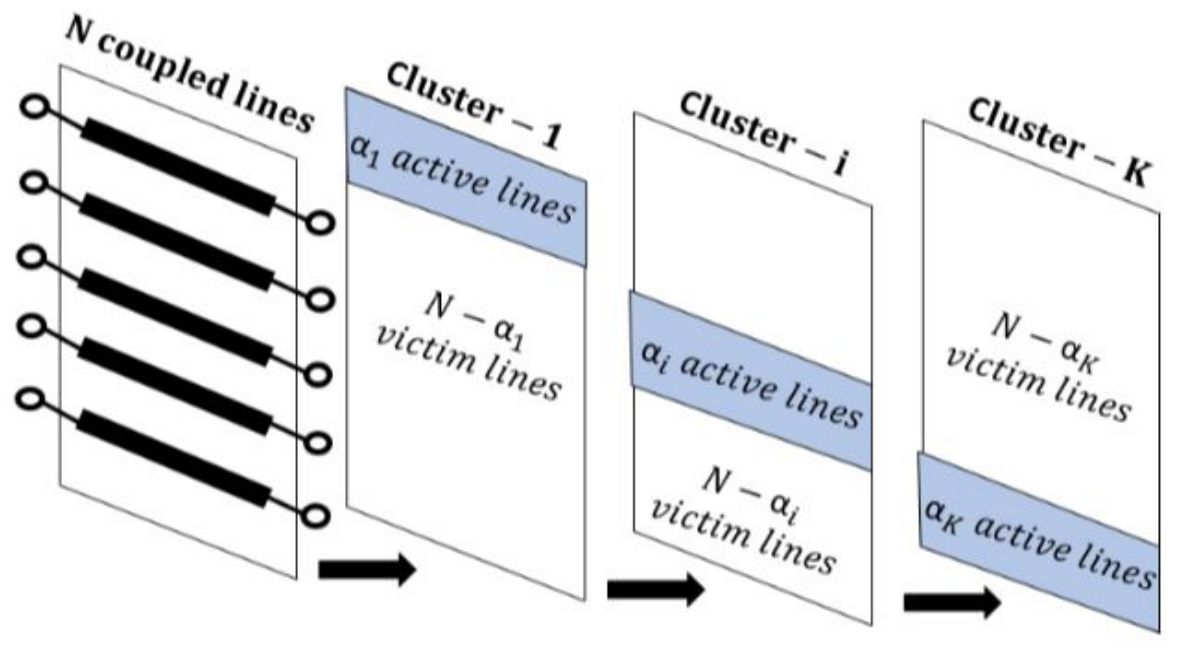

Figure 5.2: Constructing clusters in N coupled lines.

The formulation for this multi-line interconnect structure as an LTI circuit was presented in Chapter 2 and is repeated as follows for ease of reference

$$
\mathbf{C} \frac{d \mathbf{x}(t)}{d t}+\mathbf{G} \mathbf{x}(t)=\mathbf{B u}(t)
$$




$$
\mathbf{y}(t)=\mathbf{L x}(t)
$$

where $\mathbf{G}, \mathbf{C} \in \mathbb{R}^{n \times n}, \mathbf{x}(t) \in \mathbb{R}^{n}$, and $\mathbf{B} \in \mathbb{R}^{n \times n_{\text {in }}}$ and $\mathbf{L} \in \mathbb{R}^{n_{\text {out }} \times n}$ are input and output selection matrices, respectively. For the macromodeling purpose it is considered $p=$ $n_{\text {in }}=n_{\text {out }}=2 N$. The transfer function of this original (unreduced) circuit in (5.1) is

$$
\mathbf{H}(s)=\mathbf{L}(\mathbf{G}+s \mathbf{C})^{-1} \mathbf{B} \quad \in \mathbb{C}^{p \times p}
$$

where $s=\alpha+j \omega$ is the Laplace complex variable. The transfer function (5.2) at the expansion point $s_{0}$ where the matrix $G+s_{0} C$ is non-singular can be rewritten as

$$
\begin{aligned}
\mathbf{H}(s) & =\mathbf{L}\left(\mathbf{G}+s_{0} \mathbf{C}+\left(s-s_{0}\right) \mathbf{C}\right)^{-1} \mathbf{B} \\
& =\mathbf{L}\left(\mathbf{I}+\left(s-s_{0}\right) \mathbf{A}\right)^{-1} \mathbf{R}
\end{aligned}
$$

where

$$
\begin{aligned}
& \mathbf{A}=\left(\mathbf{G}+s_{0} \mathbf{C}\right)^{-1} \mathbf{C} \\
& \mathbf{R}=\left(\mathbf{G}+s_{0} \mathbf{C}\right)^{-1} \mathbf{B}
\end{aligned}
$$

From (5.3), the Taylor series expansion of the transfer function (5.2) at the expansion point $s_{0}$ is

$$
\mathbf{H}(s)=\mathbf{L} \sum_{i=0}^{\infty}(-1)^{i} \mathbf{M}_{i}\left(s_{0}\right)\left(s-s_{0}\right)^{i}
$$

where the $i$-th moment of the transfer function is

$$
\mathbf{M}_{i}\left(s_{0}\right)=\mathbf{L} \mathbf{A}^{i} \mathbf{R}
$$

Using the Arnoldi algorithm in Chapter 3, the orthogonal projection matrix $\mathbf{V}_{n \times q}$ is 
computed to match $m$ block moments as

$$
\operatorname{colsp}(\mathbf{V})=\mathcal{K}_{r}(\mathbf{A}, \mathbf{R}, m)=\operatorname{span}\left\{\mathbf{R}, \mathbf{A R}, \mathbf{A}^{2} \mathbf{R}, \ldots, \mathbf{A}^{m-1} \mathbf{R}\right\}
$$

Applying a conventional model order reduction technique such as PRIMA [48] in Chapter 3, the reduced system is obtained as

$$
\boldsymbol{\Psi}_{r}:\left\{\begin{array}{l}
\mathbf{G}_{r} \mathbf{z}(t)+\mathbf{C}_{r} \frac{d \mathbf{z}(t)}{d t}=\mathbf{B}_{r} \mathbf{u}(t) \\
\mathbf{y}(t)=\mathbf{L}_{r} \mathbf{z}(t)
\end{array}\right.
$$

where

$$
\mathbf{G}_{r}=\mathbf{V}^{\top} \mathbf{G V} \quad \mathbf{C}_{r}=\mathbf{V}^{\top} \mathbf{C V} \quad \mathbf{B}_{r}=\mathbf{V}^{\top} \mathbf{B} \quad \mathbf{L}_{r}=\mathbf{L V}
$$

It is important to note that for the massively couple transmission line structures having many input terminals, the order $q=m \times n_{\text {in }}$ can be proportionally substantial. As a result, the system matrices in (5.9) become big and dense. The simulation time for such reduced models (5.8) may easily exceed the original system. This not only undermines the advantage of applying MOR, but it may even aggravate the CPU cost. The attempt to alleviate this challenge necessitates the following treatment for the reduction of these circuits.

\subsubsection{Formulation of the Reduced-order Models based on Clustering}

Let us break down the original system (5.1) into subsystems $\boldsymbol{\Psi}_{i}, i=1,2, \ldots, K$ associated with $K$ input clusters as illustrated in (5.2). An individual cluster consists of a subset of $\beta_{i}$ active lines, i.e., the lines connected to the inputs, and $N-\beta_{i}$ victim 
lines, whose inputs are disabled. It is important to note that no two clusters can share input terminals.

Given a submodel $\boldsymbol{\Psi}_{i}$, characterized by admittance parameters [2]. Let $m_{i}$ inputs to cluster\#i be energized by the voltage sources while all the other terminals are grounded. The corresponding responses at all $2 N$ terminals are recorded. Each submodel $\boldsymbol{\Psi}_{i}$ for $i=1, \ldots, K$ can be represented as

$$
\boldsymbol{\Psi}_{i}:\left\{\begin{array}{l}
\mathbf{G x}_{i}(t)+\mathbf{C} \frac{d \mathbf{x}_{i}(t)}{d t}=\mathbf{B}_{i} \mathbf{u}_{i}(t) \\
\mathbf{y}_{i}(t)=\mathbf{L} \mathbf{x}_{i}(t)
\end{array}\right.
$$

It is to be noted that the circuit matrices $\mathbf{C}, \mathbf{G}$, and $\mathbf{L}$ are the same for all submodels, while $\mathbf{B}_{i}$ is the section of columns from the original $\mathbf{B}$ matrix corresponding to the input(s) in cluster $\# i$. So, no need to repeat the stamping process each time. Consequently, increase the calculation speed.

The next step in the reduction of the subsystems $\boldsymbol{\Psi}_{i}$ is to compute the suitable projection matrix $\mathbf{V}_{i}$ as

$$
\operatorname{colsp}\left(\mathbf{V}_{i}\right)=\mathcal{K}_{r}\left(\mathbf{A}, \mathbf{R}_{\mathbf{i}}, m\right)=\operatorname{span}\left\{\mathbf{R}_{i}, \mathbf{A} \mathbf{R}_{i}, \mathbf{A}^{2} \mathbf{R}_{i}, \ldots, \mathbf{A}^{m-1} \mathbf{R}_{i}\right\}
$$

where $\mathbf{A}$ is defined in (5.4a) and

$$
\mathbf{R}_{i}=\left(\mathbf{G}+s_{0} \mathbf{C}\right)^{-1} \mathbf{B}_{i}
$$

The reduced-order model for the cluster-\#i can be represented as:

$$
\hat{\mathbf{\Psi}}_{i}:\left\{\begin{array}{l}
\hat{\mathbf{G}}_{i}(t)+\hat{\mathbf{C}} \frac{d \mathbf{z}_{i}(t)}{d t}=\hat{\mathbf{B}}_{i} \mathbf{u}_{i}(t) \\
\hat{\mathbf{y}}_{i}(t)=\hat{\mathbf{L}} \mathbf{z}_{i}(t)
\end{array}\right.
$$


where the MNA matrices:

$$
\hat{\mathbf{G}}_{i}=\mathbf{V}_{i}^{T} \mathbf{G V}_{i} \quad \hat{\mathbf{C}}_{i}=\mathbf{V}_{i}^{T} \mathbf{C V}_{i} \quad \hat{\mathbf{B}}_{i}=\mathbf{V}_{i}^{T} \mathbf{B}_{i} \quad \hat{\mathbf{L}}_{i}=\mathbf{L V}_{i}
$$

The overall output is obtained by superposing all the responses from the individual clusters.

$$
\mathbf{y}(t) \approx \hat{\mathbf{y}}(t)=\sum_{i=1}^{K} \hat{\mathbf{y}}_{i}(t)=\sum_{i=1}^{K} \hat{\mathbf{L}}_{i} \mathbf{z}_{i}(t)=\mathbf{L} \sum_{i=1}^{K} \mathbf{V}_{i} \mathbf{z}_{i}(t)
$$

A symbolic representation for the reduced model $\boldsymbol{\Psi}_{r}$ is obtained as

$$
\mathbf{\Psi}_{r}:\left\{\begin{array}{l}
\mathbf{G}_{r} \mathbf{z}(t)+\mathbf{C}_{r} \frac{d \mathbf{z}(t)}{d t}=\mathbf{B}_{r} \mathbf{u}(t) \\
\mathbf{y}(t)=\mathbf{L}_{r} \mathbf{z}(t)
\end{array}\right.
$$

where $\mathbf{z}(t)=\left[\mathbf{z}_{1}(t), \ldots, \mathbf{z}_{K}(t)\right]^{\top}$ and the projection matrix $\mathbf{V}$ can be find by concatenating the individual projection matrix $\mathbf{V}_{i}$, i.e.,

$$
\mathbf{V}=\operatorname{blkdiag}\left(\mathbf{V}_{i}\right) \quad \text { for } \mathrm{i}=1, \ldots, \mathrm{K}
$$

Form the (5.15) and (5.16) the output matrix can be write as:

$$
\mathbf{L}_{r}=\widehat{\boldsymbol{L}} \mathbf{V}
$$

where

$$
\widehat{\boldsymbol{L}}=[1, \ldots, 1]_{1 \times K} \otimes \mathbf{L}
$$

The symbol $\otimes$ represents the Kronecker product of the matrices. At the same time, the operator blkdiag creates a block diagonal matrix with its operand matrices placed along its diagonal to form a block-diagonal matrix. Likewise, the system matrices used 
in $(5.15)$ can be defined as:

$$
\begin{array}{lr}
\mathbf{C}_{r}=\mathbf{V}^{T} \widehat{\boldsymbol{C}} \mathbf{V} & \text { where } \widehat{\boldsymbol{C}}=\mathbf{I}_{K} \otimes \mathbf{C} \\
\mathbf{G}_{r}=\mathbf{V}^{T} \widehat{\boldsymbol{G}} \mathbf{V} & \widehat{\boldsymbol{G}}=\mathbf{I}_{K} \otimes \mathbf{G} \\
\mathbf{B}_{r}=\mathbf{V}^{T} \widehat{\boldsymbol{B}} & \widehat{\boldsymbol{B}}=\operatorname{blkdiag}\left(\mathbf{B}_{i}\right)
\end{array}
$$

where matrix $\mathbf{I}_{K}$ is an $K \times K$ identity matrix. The reduced model formed using (5.16)-(5.21) has a size of " $q \times q$ " and most importantly, the system matrices $\mathbf{C}_{r}$ and $\mathbf{G}_{r}$ are sparse and block diagonal.

\subsection{Properties of Clustering Technique}

\subsubsection{Moments Preservation}

The method explained above in Section 5.3.1 preserves the first $m$ block moments of the original transfer function, the same number of moments matched by using the Arnoldi algorithm and PRIMA. To justify this hypothesis, first, the definition for "input-to-state" transfer functions as the (transfer) function relating the inputs to the circuit variables is formalized to justify this hypothesis.

- For the original (unreduced) subsystem $\boldsymbol{\Psi}_{i}$ (5.10) formed with the $i$-th cluster of input(s) $\mathbf{u}_{i}$ (where all other inputs are disabled), "input-to-state" transfer function is defined as:

$$
\mathbf{H}_{i}(s)=(\mathbf{G}+s \mathbf{C})^{-1} \mathbf{B}_{i}
$$

- The "input-to-state" transfer function of the corresponding $i$-th reduced subsystem (5.13) is

$$
\hat{\mathbf{H}}_{i}(s)=\left(\hat{\mathbf{G}}_{i}+s \hat{\mathbf{C}}_{i}\right)^{-1} \hat{\mathbf{B}}_{i}
$$


Considering the definitions in (5.22) and (5.23), the following Theorems 3 and 4 are developed.

Theorem 3. Let the $k$-th moments of the input-to-state transfer functions in (5.22) and (5.23) be $\boldsymbol{M}_{k}$ and $\widehat{\mathcal{M}} \boldsymbol{i}_{k}$, respectively. The first $\chi_{i}=\left\lfloor q_{i} / m_{i}\right\rfloor$ block moments are related (matched) as

$$
\boldsymbol{M i}_{k}=\mathbf{V}_{i} \widehat{\mathcal{M i}}_{k} \quad \text { for } k=1,2, \ldots, \chi_{i}
$$

Theorem 4. The transfer function of the reduced-order system obtained using clustering method (5.15) preserves the first $\chi$ block moments of the transfer functions of the original system $(5.15)$, where $\chi=\min _{k=1}^{K} \chi_{i}$

The proves for Theorem 3 and 4 are presented in Appendices C and D, respectively.

\subsubsection{Stability}

Stability is a crucial property that needs to be preserved when reducing the system. Consider the reduced system (5.15) with the poles as singularities of matrix pencil $\left(-\mathbf{G}_{r}, \mathbf{C}_{r}\right)$ that are obtained by solving the generalized eigenvalue problem or $-\mathbf{G}_{r} \tilde{\mathbf{X}}=\lambda \mathbf{C}_{r} \tilde{\mathbf{X}}$, where $\tilde{\mathbf{X}} \neq 0[7]$. The matrix pencil of physical systems is regular, and hence, they have a finite number of poles whose locations in the complex s-plane define the stability characteristic of the system. The necessary and sufficient condition for any macromodel to be asymptotically stable is that all the finite eigenvalues of the corresponding matrix pencil lie in the open left half-plane [82].

Theorem 5. The reduced-order system obtaining using clustering algorithm in (5.15) is asymptotically stable.

Proof. Consider the block diagonal matrix pencil of $\left(\mathbf{G}_{r}+s \mathbf{C}_{r}\right)$ from the (5.15). This 
matrix pencil can be rewrite as using (5.19) and (5.20)

$$
\left(\mathbf{G}_{r}+s \mathbf{C}_{r}\right)=\left[\begin{array}{llll}
\hat{\mathbf{G}}_{1}+s \hat{\mathbf{C}}_{1} & & & \\
& \ddots & & \\
& & & \\
& \hat{\mathbf{G}}_{i}+s \hat{\mathbf{C}}_{i} & & \\
& & \ddots & \\
& & & \\
& & & \hat{\mathbf{G}}_{K}+s \hat{\mathbf{C}}_{K}
\end{array}\right]
$$

Each diagonal element is a regular matrix pencil for the stable subsystem. The spectrum of the diagonal pencil is the union of the spectra of blocks on diagonal as:

$$
\lambda\left(\mathbf{G}_{r}+s \mathbf{C}_{r}\right)=\bigcup_{i=1}^{K} \lambda\left(\hat{\mathbf{G}}_{i}+s \hat{\mathbf{C}}_{i}\right)
$$

As a result, the spectrum of the matrix pencil of the reduced-order model is made up of the union of the complex numbers whose real parts are negative, proving that the reduced system 5.15 is asymptotically stable. 


\section{Chapter 6}

\section{Parametric Model-Order Reduce of Multi-port Networks via Clustering}

Chapter 3 presented the concept of model-order reduction and its application in handling the increasing complexity of the circuits. The essential MOR techniques, such as Krylov-subspace-based methods, were reviewed. The details of PRIMA as a wellestablished reduction method in its class were presented. We also pointed out that as the number of inputs to the circuit increases, the advantage expected by applying moment-matching-based MOR is proportionally dismissed. Chapter 4 dealt with the application of MOR to the domain of parametric circuits to track the variations in the circuit response due to variations in a selected set of design parameters. Section 4.5 in the latter chapter also pointed out that the size of parametric reduced-order models rapidly increases with the number of parameters resulting in large reduced models, which are CPU expansive to simulate. Model order reduction of multi-port circuits is a challenging task on its own, even in the absence of stochastic parameters. Chapter 5 was devoted to reviewing the state-of-the-art MOR strategy for creating efficient reduced-order macromodels of networks with a large number of input terminals. The Clustering method was presented that provides promising results for massively coupled multi-conductor interconnect networks. 
The parameterized model order reduction of multi-port circuits spawn new challenges to applying PMOR to practical problems. The resulting reduced model can quickly become impractically large for circuits with many inputs and design parameters, undermining any advantage expected from applying MOR.

This chapter presents the main contributions of the thesis. The contribution introduced by this thesis targets the application of PMOR to coupled multi-conductor interconnect networks with several design parameters. A novel algorithm is developed by adapting the PMOR incorporate with the input-clustering approach to achieve the parametric reduced model of massively coupled large parametric interconnect networks. The central idea in the proposed method is dividing the whole parametric system into subsystems and then reducing subsystems individually. Next, following the superposition paradigm, the parametric reduced subsystems are merged to obtain a parametric reduced-order model for the whole circuit. Following these steps, the reduced parametric system becomes block diagonal structured, sparse, and less CPU expensive.

It is to be noted that, since the proposed method is established based on superposition principle for general linear systems, the application of the proposed method is not limited to parameterized reduction of interconnect problems. It can be used for any general circuit.

\subsection{Parametrized Circuit Formulation}

General multi-parameter circuit equations are given in (4.9) and repeated here for ease of reference.

$$
\begin{aligned}
\left(\tilde{\mathbf{E}}_{0}+\tilde{\mathbf{E}}_{1} \tilde{\lambda}_{1}+\cdots+\tilde{\mathbf{E}}_{p} \tilde{\lambda}_{p}\right) \mathbf{X}(\boldsymbol{\lambda}) & =\mathbf{B U}(s) \\
\mathbf{Y}(\boldsymbol{\lambda}) & =\mathbf{L X}(\boldsymbol{\lambda}) .
\end{aligned}
$$


where $\tilde{\mathbf{E}}_{i}$ for $i=0, \ldots, p$ are the system matrices defined in (4.7) and $\tilde{\lambda}_{i}$, for $i=$ $1, \ldots, p$ signifies the dependency of the system response on the design parameters.

\subsection{Multi-parameter Moments of Circuit Re- sponse}

Let $\tilde{\boldsymbol{\lambda}}^{0}=\left[\tilde{\lambda}_{1}^{0}, \tilde{\lambda}_{2}^{0}, \ldots, \tilde{\lambda}_{p}^{0}\right]^{\top}$ be selected such that the circuit response can be characterized at $\tilde{\boldsymbol{\lambda}}^{0}$. From (6.1a) it is straightforward to see that the only assumption on $\tilde{\boldsymbol{\lambda}}^{0}$ is that the matrix $\left(\tilde{\mathbf{E}}_{0}+\tilde{\lambda}_{1}^{0} \tilde{\mathbf{E}}_{1}+\cdots+\tilde{\lambda}_{p}^{0} \tilde{\mathbf{E}}_{p}\right)$ be nonsingular. This assumption qualifies $\tilde{\lambda}^{0}$ as an expansion point for the Taylor series expansion of the circuit response.

Using a variable shift,

$$
\sigma \triangleq \tilde{\lambda}-\tilde{\lambda}^{0}
$$

where

$$
\sigma_{i} \triangleq \tilde{\lambda}_{i}-\tilde{\lambda}_{i}^{0}, \quad \text { for } i=1, \ldots, p
$$

the response of the parametric system in (6.1) can be written as

$$
\begin{aligned}
\mathbf{X}(\tilde{\boldsymbol{\lambda}}) & =\left(\tilde{\mathbf{E}}_{0}+\tilde{\mathbf{E}}_{1} \tilde{\lambda}_{1}+\cdots+\tilde{\mathbf{E}}_{p} \tilde{\lambda}_{p}\right)^{-1} \mathbf{B U}(s) \\
& =(\underbrace{\tilde{\mathbf{E}}_{0}+\tilde{\mathbf{E}}_{1} \tilde{\lambda}_{1}^{0}+\cdots+\tilde{\mathbf{E}}_{p} \tilde{\lambda}_{p}^{0}}_{\tilde{\mathbf{E}}}+\tilde{\mathbf{E}}_{1}\left(\tilde{\lambda}_{1}-\tilde{\lambda}_{1}^{0}\right)+\cdots+\tilde{\mathbf{E}}_{p}\left(\tilde{\lambda}_{p}-\tilde{\lambda}_{p}^{0}\right))^{-1} \mathbf{B U}(s) \\
& =\left(\mathbf{I}+\tilde{\mathbf{E}}^{-1} \tilde{\mathbf{E}}_{1}\left(\tilde{\lambda}_{1}-\tilde{\lambda}_{1}^{0}\right)+\cdots+\tilde{\mathbf{E}}^{-1} \tilde{\mathbf{E}}_{p}\left(\tilde{\lambda}_{p}-\tilde{\lambda}_{p}^{0}\right)\right)^{-1} \tilde{\mathbf{E}}^{-1} \mathbf{B U}(s)
\end{aligned}
$$

Equivalently, it is

$$
\mathbf{X}(\tilde{\boldsymbol{\lambda}})=\left(\mathbf{I}-\left(\sigma_{1} \mathbf{M}_{1}+\cdots+\sigma_{p} \mathbf{M}_{p}\right)\right)^{-1} \mathbf{B}_{M}
$$


where

$$
\begin{aligned}
\tilde{\mathbf{E}} & =\tilde{\mathbf{E}}_{0}+\tilde{\lambda}_{1}^{0} \tilde{\mathbf{E}}_{1}+\cdots+\tilde{\lambda}_{p}^{0} \tilde{\mathbf{E}}_{p} \\
\mathbf{B}_{M} & =\tilde{\mathbf{E}}^{-1} \mathbf{B} \\
\mathbf{M}_{i} & =-\tilde{\mathbf{E}}^{-1} \tilde{\mathbf{E}}_{i} \quad i=1,2, \ldots, p
\end{aligned}
$$

Following the steps elaborated in Section 4.1.1, the Taylor series expansion of the (6.5) at $\boldsymbol{\sigma}=\mathbf{0}$ is

$$
\begin{aligned}
& \mathbf{X}(\tilde{\boldsymbol{\lambda}})=\sum_{m=0}^{\infty}\left(\sigma_{1} \mathbf{M}_{1}+\cdots+\sigma_{p} \mathbf{M}_{p}\right)^{m} \mathbf{B}_{M}= \\
& \sum_{m=0}^{\infty} \sum_{k_{2}=0}^{m-\left(k_{3}+\cdots+k_{p}\right)} \cdots \sum_{k_{p-1}=0}^{m-k_{p}} \sum_{k_{p}=0}^{m} \mathbf{F}_{k_{2}, \ldots, k_{p}}^{m}\left(\mathbf{M}_{1}, \ldots, \mathbf{M}_{p}\right) \mathbf{B}_{M} \sigma_{1}^{m-\left(k_{2}+\cdots+k_{p}\right)} \sigma_{2}^{k_{2}} \ldots \sigma_{p}^{k_{p}}
\end{aligned}
$$

where

$$
\mathbf{F}_{k_{2}, \ldots, k_{p}}^{m}\left(\mathbf{M}_{1}, \ldots, \mathbf{M}_{p}\right)= \begin{cases}0, & \text { if } k_{i} \notin\{0,1, \ldots, m\}, \quad i=2, \ldots, p \\ 0, & \text { if } k_{2}+\cdots+k_{p} \notin\{0,1, \ldots, m\} \\ \mathbf{I}_{n} & \text { if } m=0 \\ \mathbf{M}_{1} \mathbf{F}_{k_{2}, \ldots, k_{p}}^{m-1}\left(\mathbf{M}_{1}, \ldots, \mathbf{M}_{p}\right)+\mathbf{M}_{2} \mathbf{F}_{k_{2}-1, \ldots, k_{p}}^{m-1}\left(\mathbf{M}_{1}, \ldots\right. \\ \left.\mathbf{M}_{p}\right)+\cdots+\mathbf{M}_{p} \mathbf{F}_{k_{2}, \ldots, k_{p}-1}^{m-1}\left(\mathbf{M}_{1}, \ldots, \mathbf{M}_{p}\right) \\ \vdots\end{cases}
$$

Example. Given that there are two parameters as $\sigma_{1}$ and $\sigma_{2}$, the moments of the parametric circuit response evaluated at $\boldsymbol{\sigma}=\mathbf{0}$ are computed.

To construct the moments, one should first compute the following coefficient matrices. 


$$
\begin{aligned}
& \mathbf{F}_{k_{2}, \ldots, k_{p}}^{m}\left(\mathbf{M}_{1}, \ldots, \mathbf{M}_{p}\right)=\mathbf{F}_{k_{2}}^{m}\left(\mathbf{M}_{1}, \ldots, \mathbf{M}_{p}\right)= \\
& \left\{\begin{array}{l}
\mathbf{F}_{0}^{0}=\mathbf{I} \\
\mathbf{F}_{0}^{1}=\mathbf{M}_{1} \mathbf{F}_{0}^{0}=\mathbf{M}_{1}, \quad \mathbf{F}_{1}^{1}=\mathbf{M}_{2} \mathbf{F}_{0}^{0}=\mathbf{M}_{2}, \text { Coefficients of } 1 \text {-st order terms } \\
\mathbf{F}_{0}^{2}=\mathbf{M}_{1} \mathbf{F}_{0}^{1}=\left(\mathbf{M}_{1}\right)^{2}, \quad \mathbf{F}_{1}^{2}=\mathbf{M}_{1} \mathbf{F}_{1}^{1}+\mathbf{M}_{2} \mathbf{F}_{0}^{1}=\mathbf{M}_{1} \mathbf{M}_{2}+\mathbf{M}_{2} \mathbf{M}_{1}, \\
\mathbf{F}_{2}^{2}=\mathbf{M}_{2} \mathbf{F}_{1}^{1}=\left(\mathbf{M}_{2}\right)^{2}, \\
\vdots
\end{array}\right.
\end{aligned}
$$

Then, the moments are obtained denoted as $\mathbf{N}_{k}^{m}$, where the superscript $m$ is the moment's order and the index $k$ is the count,

The zero-order moment:

$$
\mathbf{N}_{1}^{0}=\mathbf{F}_{0}^{0} \mathbf{B}_{M}=\mathbf{B}_{M}
$$

The first-order moments:

$$
\mathbf{N}_{1}^{1}=\mathbf{M}_{1} \mathbf{B}_{M} \quad \mathbf{N}_{2}^{1}=\mathbf{M}_{2} \mathbf{B}_{M}
$$

The second-order moments:

$$
\mathbf{N}_{1}^{2}=\left(\mathbf{M}_{1}\right)^{2} \mathbf{B}_{M} \quad \mathbf{N}_{2}^{2}=\left(\mathbf{M}_{2}\right)^{2} \mathbf{B}_{M} \quad \mathbf{N}_{3}^{2}=\left(\mathbf{M}_{1} \mathbf{M}_{2}+\mathbf{M}_{2} \mathbf{M}_{1}\right) \mathbf{B}_{M}
$$

For the general case, having parameters as $\sigma_{1}, \sigma_{2}, \ldots, \sigma_{p}$, the moment matrices are constructed as

The zero-order moment $\left(\mathrm{N}_{1}^{0}\right)$ :

$$
\mathbf{N}_{1}^{0}=\mathbf{B}_{M}
$$


The first-order moments $\left(\mathbf{N}_{i}^{1}\right.$ for $\left.i=1, \ldots, p\right)$ :

$$
\mathbf{N}_{i}^{1}=\mathbf{M}_{i} \mathbf{B}_{M} \quad \text { for } i=1, \ldots, p
$$

The second-order moments $\left(\mathbf{N}_{i}^{2}\right.$ for $\left.i=1, \ldots, p_{2}\right)$ :

$$
\begin{aligned}
& \mathbf{N}_{i}^{2}=\left(\mathbf{M}_{i}\right)^{2} \mathbf{B}_{M} \quad \text { for } i=1, \ldots, p \\
& \mathbf{N}_{j}^{2}=\left(\mathbf{M}_{1} \mathbf{M}_{i}+\mathbf{M}_{i} \mathbf{M}_{1}\right) \mathbf{B}_{M} \quad \text { for } i=2, \ldots, p ; \quad j=(p-1)+i \\
& \mathbf{N}_{k}^{2}=\left(\mathbf{M}_{2} \mathbf{M}_{i}+\mathbf{M}_{i} \mathbf{M}_{2}\right) \mathbf{B}_{M} \quad \text { for } i=3, \ldots, p ; \quad k=(2 p-3)+i \\
& \mathbf{N}_{p_{2}}^{2}=\left(\mathbf{M}_{p-1} \mathbf{M}_{p}+\mathbf{M}_{p} \mathbf{M}_{p-1}\right) \mathbf{B}_{M}
\end{aligned}
$$

where the first order moments $\mathbf{N}_{i}^{1}$ are the coefficients of $\sigma_{i}$, for $i=1, \ldots, p$, the second order moments $\mathbf{N}_{i}^{2}$ are the coefficients of $\sigma_{i}^{2}$, for $i=1, \ldots, p, \mathbf{N}_{j}^{2}$ are the coefficients of $\sigma_{1} \sigma_{i}$, for $i=2, \ldots, p, \mathbf{N}_{k}^{2}$ are the coefficients of $\sigma_{2} \sigma_{i}$, for $i=3, \ldots, p, \ldots$, and $\mathbf{N}_{l}^{2}$ is the coefficient of $\sigma_{p-1} \sigma_{p}$.

The subspace of the multi-parameter moments (a.k.a. Krylov subspace) for a general parametric circuit can be defined as the space spanned by the columns in the moment matrices. The (orthonormal) basis spanning this subspace used as the projection matrix $\mathbf{V}$,

$$
\begin{array}{r}
\operatorname{colsp}(\mathbf{V})=\operatorname{colsp}\left(\left[\mathbf{B}_{M}, \mathbf{M}_{1} \mathbf{B}_{M}, \ldots, \mathbf{M}_{p} \mathbf{B}_{M},\left(\mathbf{M}_{1}\right)^{2} \mathbf{B}_{M}, \ldots,\left(\mathbf{M}_{p}\right)^{2} \mathbf{B}_{M}\right.\right. \\
\left(\mathbf{M}_{1} \mathbf{M}_{2}+\mathbf{M}_{2} \mathbf{M}_{1}\right) \mathbf{B}_{M}, \ldots,\left(\mathbf{M}_{1} \mathbf{M}_{p}+\mathbf{M}_{p} \mathbf{M}_{1}\right) \mathbf{B}_{M},\left(\mathbf{M}_{2} \mathbf{M}_{3}+\mathbf{M}_{3} \mathbf{M}_{2}\right) \mathbf{B}_{M}, \ldots \\
\left.\left.\left(\mathbf{M}_{2} \mathbf{M}_{p}+\mathbf{M}_{p} \mathbf{M}_{2}\right) \mathbf{B}_{M}, \ldots,\left(\mathbf{M}_{p-1} \mathbf{M}_{p}+\mathbf{M}_{p} \mathbf{M}_{p-1}\right) \mathbf{B}_{M}, \ldots\right]\right)
\end{array}
$$


The moments in (6.18) spanning circuit's Krylov subspace

The form of the circuits' multi-parameter moments spanning their Krylov subspace allows an implicit moment matching using an implicit recursive modified Gram-Schmidt-like process to construct a projection matrix V [53]. The principles and the implementable algorithm for this approach have been explored in Chapter 4 .

\subsection{Parametric Reduced-Order Model}

Using the projection matrix $\mathbf{V}$ in (6.18) and the reduction scheme in Chapter 4, the parametric reduced-order system for the original circuit in (6.1) is obtained as discussed in (4.19) and repeated here for ease of reference.

$$
\begin{aligned}
\left(\hat{\mathbf{E}}_{0}+\hat{\mathbf{E}}_{1} \tilde{\lambda}_{1}+\cdots+\hat{\mathbf{E}}_{p} \tilde{\lambda}_{p}\right) \mathbf{Z}(\tilde{\boldsymbol{\lambda}}) & =\hat{\mathbf{B}} \mathbf{U}(s) \\
\mathbf{Y}(\tilde{\boldsymbol{\lambda}}) & =\hat{\mathbf{L}} \mathbf{Z}(\tilde{\boldsymbol{\lambda}}) .
\end{aligned}
$$

where

$$
\begin{array}{rrrr}
\hat{\mathbf{E}}_{0} \triangleq \mathbf{V}^{\top} \tilde{\mathbf{E}}_{0} \mathbf{V} & \hat{\mathbf{E}}_{1} \triangleq \mathbf{V}^{\top} \tilde{\mathbf{E}}_{1} \mathbf{V} & \ldots & \hat{\mathbf{E}}_{p} \triangleq \mathbf{V}^{\top} \tilde{\mathbf{E}}_{p} \mathbf{V} \\
\hat{\mathbf{B}} \triangleq \mathbf{V}^{\top} \mathbf{B} & \hat{\mathbf{L}} \triangleq \mathbf{L V} & \text { and } & \mathbf{Z}(\tilde{\boldsymbol{\lambda}}) \triangleq \mathbf{V}^{\top} \mathbf{X}(\boldsymbol{\lambda})
\end{array}
$$

The form of the moments evidenced the important fact about the size of the resulting parametric reduced model (6.19) highlighted earlier. Considering (6.18) it is straightforward to see that to match the first multi-parameter moments up to a particular order, the number of bases spanning the moment-space and hence the size of the resulting reduced model is proportional to the number of circuits' inputs. The order of reduced models for multi-parameter problems is generally large, making the 
parametric order-reduction a challenging task. The fact that the order also proportionally increases with the number of ports severely aggravates the challenge. The rest of this chapter is devoted to resolving this critical barrier.

\subsection{Reduction of Parametric System via Cluster- ing}

By pushing the idea of clustering explored in Chapter 5 to the domain of parametric MOR, we can break down this original parametric system (6.1) into $K$ clusters. Each cluster comprises a subset of $\beta$ active lines, i.e., lines connected to inputs and $N-\beta$ victim lines, i.e., lines whose inputs are disabled. One important thing to note for the simplicity of the formulation is that no two clusters can have the same active line. The parametrized circuit equations representing the $i$-th subcircuit are

$$
\boldsymbol{\Pi}_{i}:\left\{\begin{array}{l}
{\left[\tilde{\mathbf{E}}_{0}+\tilde{\lambda}_{1} \tilde{\mathbf{E}}_{1}+\cdots+\tilde{\lambda}_{p} \tilde{\mathbf{E}}_{p}\right] \mathbf{X}_{i}(\tilde{\boldsymbol{\lambda}})=\mathbf{B}_{i} \mathbf{U}_{i}(s),} \\
\mathbf{Y}_{i}(\tilde{\boldsymbol{\lambda}})=\mathbf{L} \mathbf{X}_{i}(\tilde{\boldsymbol{\lambda}}) .
\end{array}\right.
$$

Note that the $\tilde{\mathbf{E}}_{i}, i=0, \ldots, p$ and $\mathbf{L}$ matrices are the same for all submodels, while $\mathbf{B}_{i}$ is the section of columns from the original $\mathbf{B}$ matrix. There is no need to repeat the stamping process each time. This fact improves the model generation cost parsimony.

Next, the individual subsystems are reduced as discussed in Section 6.3, resulting in

$$
\hat{\mathbf{\Pi}}_{i}:\left\{\begin{array}{l}
{\left[\hat{\mathbf{E}}_{0}+\tilde{\lambda}_{1} \hat{\mathbf{E}}_{1}+\cdots+\tilde{\lambda}_{p} \hat{\mathbf{E}}_{p}\right] \mathbf{Z}_{i}(\tilde{\boldsymbol{\lambda}})=\hat{\mathbf{B}}_{i} \mathbf{U}_{i}(s),} \\
\hat{\mathbf{Y}}_{i}(\tilde{\boldsymbol{\lambda}})=\hat{\mathbf{L}} \mathbf{Z}_{i}(\tilde{\boldsymbol{\lambda}}) .
\end{array}\right.
$$


where for the $i$-th subsystem $(i=1, \ldots, K)$, it is

$$
\begin{array}{cc}
\hat{\mathbf{E}}_{i, j}=\mathbf{V}_{i}^{\top} \tilde{\mathbf{E}}_{j} \mathbf{V}_{i} & j=1, \ldots, p \\
\hat{\mathbf{B}}_{i}=\mathbf{V}_{i}^{\top} \mathbf{B}_{i} & \hat{\mathbf{L}}_{i}=\mathbf{L} \mathbf{V}_{i}
\end{array}
$$

where $\mathbf{V}_{i}$ is the reduction-projection matrix calculated using the moments of $\boldsymbol{\Pi}_{i}$ (the $i$-th subsystem) obtained as explained in Section 6.2.

The overall response of the reduced system is computed by superposing the responses from the clusters,

$$
\mathbf{Y}(\tilde{\boldsymbol{\lambda}}) \approx \widehat{\boldsymbol{Y}}(\tilde{\boldsymbol{\lambda}})=\sum_{i=1}^{K} \hat{\mathbf{Y}}_{i}(\tilde{\boldsymbol{\lambda}})=\sum_{i=1}^{K} \hat{\mathbf{L}}_{i} \mathbf{Z}_{i}(\tilde{\boldsymbol{\lambda}})=\mathbf{L} \sum_{i=1}^{K} \mathbf{V}_{i} \mathbf{Z}_{i}(\tilde{\boldsymbol{\lambda}})
$$

The overall parametric reduced system $\widehat{\Pi}$ is constituted through a systematic aggregation of the parametric reduced subsystems $\hat{\boldsymbol{\Pi}}_{i}, i=1, \ldots, K$ as

$$
\widehat{\Pi}:\left\{\begin{array}{l}
{\left[\widehat{\boldsymbol{E}}_{0}+\tilde{\lambda}_{1} \widehat{\boldsymbol{E}}_{1}+\cdots+\tilde{\lambda}_{p} \widehat{\boldsymbol{E}}_{p}\right] \widehat{\boldsymbol{Z}}(\tilde{\boldsymbol{\lambda}})=\widehat{\boldsymbol{B}} \mathbf{U}(s)} \\
\widehat{\boldsymbol{Y}}(\tilde{\boldsymbol{\lambda}})=\widehat{\boldsymbol{L}} \widehat{\boldsymbol{Z}}(\tilde{\boldsymbol{\lambda}})
\end{array}\right.
$$

where $\widehat{\boldsymbol{Z}}(\tilde{\boldsymbol{\lambda}})=\left[\mathbf{Z}_{1}(\tilde{\boldsymbol{\lambda}}), \ldots, \mathbf{Z}_{K}(\tilde{\boldsymbol{\lambda}})\right]^{\top}$ and the projection matrix $\widehat{\boldsymbol{V}}$ is found by concatenating the individual projection matrix $\mathbf{V}_{i}$

$$
\begin{aligned}
\widehat{\boldsymbol{V}} & =\operatorname{blkdiag}\left(\left[\mathbf{V}_{1}, \ldots, \mathbf{V}_{K}\right]\right) \\
& =\left[\begin{array}{lll}
\mathbf{V}_{1} & & \\
& \ddots & \\
& & \\
& & \mathbf{V}_{k}
\end{array}\right]
\end{aligned}
$$


Considering the (6.25) and (6.26), The input matrix for the reduced overall model is

$$
\widehat{\boldsymbol{B}}=\widehat{\boldsymbol{V}}^{\top} \overline{\mathbf{B}}
$$

where

$$
\overline{\mathbf{B}}=\operatorname{blkdiag}\left(\left[\mathbf{B}_{1}, \ldots, \mathbf{B}_{K}\right]\right)
$$

the output matrix is

$$
\widehat{\boldsymbol{L}}=\overline{\mathbf{L}} \widehat{\boldsymbol{V}}
$$

where

$$
\overline{\mathbf{L}}=[1, \ldots, 1]_{1 \times K} \otimes \mathbf{L}
$$

and symbol $\otimes$ is the Kronecker product of matrices.

For the $i$-th subsystem $(i=1, \ldots, K)$, the system matrices used in 6.25 are formed as

$$
\widehat{\boldsymbol{E}}_{j}=\widehat{\boldsymbol{V}}^{\top} \overline{\mathbf{E}}_{j} \widehat{\boldsymbol{V}} \quad j=1, \ldots, p
$$

where

$$
\overline{\mathbf{E}}_{i}=\mathbf{I}_{K \times K} \otimes \tilde{\mathbf{E}}_{i} \quad i=1, \ldots, K
$$

where matrix $\mathbf{I}_{K \times K}$ is an $K \times K$ identity matrix.

The resulting model in (6.25) with the matrices in (6.26)-(6.32) is a parametric reduced model with spares and block diagonal structured system matrices. These properties noticeably improve the efficiency of the reduced model and CPU cost for the simulation. In comparison, the model generation time will remain almost the same as the conventional models $[53,54]$. 


\subsection{Properties of the Proposed Algorithm}

\subsubsection{Multi-Moment Preservation}

The important property of the proposed method is moment preservation that ensures the accuracy of the reduced system. Given the multi-parameter moments of the original system $\mathbf{N}_{j}^{l}$ defined in (6.15), (6.16), (6.17), etc., and their counterparts from the parametric reduced-model from the proposed method denoted as $\widehat{\boldsymbol{N}}_{j}^{l}$, the following Theorem 6 is established.

Theorem 6 (Multi-Moment Preservation). The transfer function of the parametric reduced-order model of order $q$ in (6.25) preserves first $m=\left\lfloor\frac{q}{n_{\text {in }}}\right\rfloor$ block moments of the original parametrized system (6.1) as

$$
\begin{array}{r}
\mathbf{L}\left[\mathbf{N}_{1}^{0}, \mathbf{N}_{1}^{1}, \ldots, \mathbf{N}_{p}^{1}, \mathbf{N}_{1}^{2}, \ldots, \mathbf{N}_{p_{2}}^{2}, \mathbf{N}_{1}^{3}, \ldots, \mathbf{N}_{p_{3}}^{3}, \ldots, \mathbf{N}_{1}^{k}, \ldots, \mathbf{N}_{p_{k}}^{k}\right]= \\
\widehat{\boldsymbol{L}}\left[\widehat{\mathbf{N}}_{1}^{0}, \widehat{\boldsymbol{N}}_{1}^{1}, \ldots, \widehat{\mathbf{N}}_{p}^{1}, \widehat{\mathbf{N}}_{1}^{2}, \ldots, \widehat{\mathbf{N}}_{p_{2}}^{2}, \widehat{\boldsymbol{N}}_{1}^{3}, \ldots, \widehat{\mathbf{N}}_{p_{3}}^{3}, \ldots, \widehat{\mathbf{N}}_{1}^{k}, \ldots, \widehat{\mathbf{N}}_{p_{k}}^{k}\right] \\
\text { for } 1+p+p_{2}+\cdots+p_{k}=m
\end{array}
$$

Proof. To prove this theorem, it is important to primarily consider that the first $m$ block moments of the $i$-th original (unreduced) subsystems (for $i=1, \ldots, K$ ) in (6.20), denoted as $\mathbf{N} i_{j}^{l}, i=1, \ldots, K$, are matching with the ones of the corresponding parametric reduced-order models in (6.21), denoted as $\hat{\mathbf{N}} i_{j}^{l}$, for $i=1, \ldots, K$. It is straightforward to prove the above moment matching property in (6.34) by following 
the steps of the proof presented in $[53,54]$. Hence, we have

$$
\begin{array}{r}
{\left[\mathbf{N} i_{1}^{0}, \mathbf{N} i_{1}^{1}, \ldots, \mathbf{N} i_{p}^{1}, \mathbf{N} i_{1}^{2}, \ldots, \mathbf{N} i_{p_{2}}^{2}, \mathbf{N} i_{1}^{3}, \ldots, \mathbf{N} i_{p_{3}}^{3}, \mathbf{N} i_{1}^{k}, \ldots, \mathbf{N} i_{1}^{k} \ldots, \mathbf{N} i_{p_{k}}^{k}\right]=} \\
\mathbf{V}_{i}\left[\hat{\mathbf{N}} i_{1}^{0}, \hat{\mathbf{N}} i_{1}^{1}, \ldots, \hat{\mathbf{N}} i_{p}^{1}, \hat{\mathbf{N}} i_{1}^{2}, \ldots, \hat{\mathbf{N}} i_{p_{2}}^{2}, \hat{\mathbf{N}} i_{1}^{3}, \ldots, \hat{\mathbf{N}} i_{p_{3}}^{3}, \ldots, \hat{\mathbf{N}} i_{1}^{k}, \ldots, \hat{\mathbf{N}} i_{p_{k}}^{k}\right] \\
\text { for } 1+p+p_{2}+\cdots+p_{k}=m \\
\text { for } i=1, \ldots, K
\end{array}
$$

Next, it is to be noted that the overall response for the unreduced clustered system consisting of $k$ subsystems can be obtained by superposing the responses from $k$ subsystems as

$$
\mathbf{Y}=\mathbf{L X}=\mathbf{L}\left(\mathbf{X}_{1}+\cdots+\mathbf{X}_{K}\right)=[\mathbf{L}, \mathbf{L}, \ldots, \mathbf{L}]\left[\begin{array}{c}
\mathbf{X}_{1} \\
\mathbf{X}_{2} \\
\vdots \\
\\
\mathbf{X}_{K}
\end{array}\right]=\overline{\mathbf{L}}\left[\begin{array}{c}
\mathbf{X}_{1} \\
\mathbf{X}_{2} \\
\vdots \\
\mathbf{X}_{K}
\end{array}\right]
$$

From (6.35), it is

$$
\mathbf{L X}=\overline{\mathbf{L}}\left[\begin{array}{c}
\mathbf{X}_{1} \\
\mathbf{X}_{2} \\
\vdots \\
\mathbf{X}_{K}
\end{array}\right]
$$

By equating the coefficients of the corresponding terms in the Taylor series expansion for the both sides of (6.36), for any of the first $m$ block moments listed in (6.34), we 
have

$$
\mathbf{L} \mathbf{N}_{j}^{l}=\overline{\mathbf{L}}\left[\begin{array}{c}
\mathbf{N} 1_{j}^{l} \\
\mathbf{N} 2_{j}^{l} \\
\vdots \\
\mathbf{N} K_{j}^{l}
\end{array}\right]
$$

Considering (6.34), from (6.37), we get

$$
\mathbf{L} \mathbf{N}_{j}^{l}=\overline{\mathbf{L}}\left[\begin{array}{c}
\mathbf{V}_{1} \hat{\mathbf{N}} 1_{j}^{l} \\
\mathbf{V}_{2} \hat{\mathbf{N}} 2_{j}^{l} \\
\vdots \\
\mathbf{V}_{K} \hat{\mathbf{N}} K_{j}^{l}
\end{array}\right]=\overline{\mathbf{L}}\left[\begin{array}{ccc}
\mathbf{V}_{1} & & \\
& \mathbf{V}_{2} & \\
& \ddots & \\
& & \\
& & \mathbf{V}_{K}
\end{array}\right]\left[\begin{array}{c}
\hat{\mathbf{N}} 1_{j}^{l} \\
\hat{\mathbf{N}} 2_{j}^{l} \\
\vdots \\
\hat{\mathbf{N}} K_{j}^{l}
\end{array}\right]=\overline{\mathbf{L}} \widehat{\boldsymbol{V}}\left[\begin{array}{c}
\hat{\mathbf{N}} 1_{j}^{l} \\
\hat{\mathbf{N}} 2_{j}^{l} \\
\vdots \\
\hat{\mathbf{N}} K_{j}^{l}
\end{array}\right]
$$

From (6.38), it is concluded

$$
\mathbf{L N}_{j}^{l}=\overline{\mathbf{L}} \widehat{\boldsymbol{V}} \widehat{\mathbf{N}}_{j}^{l}
$$

A similar proof is applicable to demonstrate the equivalency in (6.33) for any of the first $m$ moments. This concludes the proof.

For simplicity, let consider $\tilde{\lambda}_{1}$ as time variable $t$ (or complex frequency $s$ in the Laplace domain). Hence, the proposed parametric reduced model(6.25) is equivalently rewritten as:

$$
\widehat{\boldsymbol{\Pi}}:\left\{\begin{array}{l}
{\left[\widehat{\boldsymbol{E}}_{0}+s \widehat{\boldsymbol{E}}_{1}+\tilde{\lambda}_{2} \widehat{\boldsymbol{E}}_{2}+\cdots+\tilde{\lambda}_{p} \widehat{\boldsymbol{E}}_{p}\right] \widehat{\boldsymbol{Z}}(\tilde{\boldsymbol{\lambda}})=\widehat{\boldsymbol{B}} \mathbf{U}} \\
\widehat{\boldsymbol{Y}}(\tilde{\boldsymbol{\lambda}})=\widehat{\boldsymbol{L}} \widehat{\boldsymbol{Z}}(\tilde{\boldsymbol{\lambda}})
\end{array}\right.
$$




\subsubsection{Stability Preservation}

One of the major properties that a reduced system should preserve is stability. As it was explored in Section 5.4.2, for the reduced system represent in (6.40), the poles of this system are located where the kernel $\left(\widehat{\boldsymbol{E}}_{0}+s \widehat{\boldsymbol{E}}_{1}+\tilde{\lambda}_{2} \widehat{\boldsymbol{E}}_{2} \cdots+\tilde{\lambda}_{p} \widehat{\boldsymbol{E}}_{p}\right)^{-1}$ is singular. As a result, the stability of the reduced system can be determined by observing the spectrum of matrix pencil $\left(-\left(\widehat{\boldsymbol{E}}_{0}+\tilde{\lambda}_{2} \widehat{\boldsymbol{E}}_{2}+\cdots+\tilde{\lambda}_{p} \widehat{\boldsymbol{E}}_{p}\right), \widehat{\boldsymbol{E}}_{1}\right)$. For the physical systems, the reduced model and its matrix pencil are singular only for finite number of values of $s \in \mathbb{C}$. This singularity can be solved by generalized eigenvalue problem as $\left(\widehat{\boldsymbol{E}}_{0}+\tilde{\lambda}_{2} \widehat{\boldsymbol{E}}_{2}+\cdots+\tilde{\lambda}_{p} \widehat{\boldsymbol{E}}_{p}\right) \mathbf{T}=-p \widehat{\boldsymbol{E}}_{1} \mathbf{T}$, where $\mathbf{T} \neq \mathbf{0}$ and $p$ is the eigenvalue [7]. For any macromodel, the necessary and sufficient condition to be asymptotically stable is that all its finite eigenvalues of the corresponding matrix pencil lie in the open left half-plane [82].

Theorem 7 (Stability). If the PMOR scheme applied to each subsystem preserves the asymptotic stability, the resulting parametric reduced-model for the overall system resulting from the proposed methodology (6.40) is asymptotically stable.

Proof. From (6.29) and (6.31), the following equations show the construction of this diagonalized reduced model

$$
\begin{aligned}
& \left(\widehat{\boldsymbol{E}}_{0}+\tilde{\lambda}_{2} \widehat{\boldsymbol{E}}_{2}+\cdots+\tilde{\lambda}_{p} \widehat{\boldsymbol{E}}_{p}+s \widehat{\boldsymbol{E}}_{1}\right)= \\
& {\left[\begin{array}{lll}
\left(\hat{\mathbf{E}}_{i, 0}+\cdots+\tilde{\lambda}_{i, p} \hat{\mathbf{E}}_{i, p}+s \hat{\mathbf{E}}_{i, 1}\right)_{i=1} & & \\
& \ddots & \\
& & \\
& & \left(\hat{\mathbf{E}}_{i, 0}+\cdots+\tilde{\lambda}_{i, p} \hat{\mathbf{E}}_{i, p}+s \hat{\mathbf{E}}_{1}\right)_{i=K}
\end{array}\right]}
\end{aligned}
$$

At every point in the parameters space defined by a set of parameters $\left(\tilde{\lambda}_{2}, \tilde{\lambda}_{3}, \ldots, \tilde{\lambda}_{p}\right)$, the spectrum of overall reduced system is the union of the spectra of blocks on diagonal 
as

$$
\lambda\left(\widehat{\boldsymbol{E}}_{0}+\cdots+\tilde{\lambda}_{2} \widehat{\boldsymbol{E}}_{2}+\tilde{\lambda}_{p} \widehat{\boldsymbol{E}}_{p}+s \widehat{\boldsymbol{E}}_{1}\right)=\bigcup_{i=1}^{K} \lambda\left(\hat{\mathbf{E}}_{i, 0}+\tilde{\lambda}_{2} \hat{\mathbf{E}}_{i, 2}+\cdots+\tilde{\lambda}_{p} \hat{\mathbf{E}}_{i, p}+s \hat{\mathbf{E}}_{i, 1}\right)_{i}
$$

where the operator $\lambda(\cdot)$ here denotes the spectrum of its operand matrix. That means the reduced system's spectrum comprises the union of the complex numbers whose real parts are negative. Hence, the overall reduced system (6.25) using the proposed method is guaranteed asymptotically stable. 


\section{Chapter 7}

\section{Computational Results}

This chapter presents two examples to demonstrate the accuracy and efficiency of the proposed method. The CPU times reported here correspond to a PC environment with $4 \mathrm{GHz}$ RAM and $1.80 \mathrm{GHz}$ Intel Core-i3 processor, executed in the MATLAB ${ }^{\circledR}$ 7.11.0 (R2010b) environment.

\subsection{Example 1}

In this example, an interconnect structure with four coupled lines in $10 \mathrm{~cm}$ length is considered as an eight-ports network ( shown in Fig. 7.1). The interconnect structure is modeled using 250 sections of lumped RLGC segmentation [83]. Three stochastic parameters are taken into considerations as widths (W) of traces, spacing (d) between the lines, and the conductivity of material $(\sigma)$. The nominal values of the stochastic physical parameters are $\mathrm{W}=1.8 \mathrm{~mm}, \mathrm{~d}=26.2 \mathrm{~mm}$, and $\sigma=18.7 \times 10^{6} \mathrm{~S} / \mathrm{m}$ are assumed to have Gaussian distribution with a standard deviation of $\pm 10 \%$.

The number of the circuit variables is 6022 , which includes node voltages and branch currents. Thus, the size of the MNA matrices of the original network constructed for the Multi-Transmission Line (MTL) structure is $6022 \times 6022$. The pro-

jection matrix for the proposed method $\mathbf{V} \in \mathbb{R}^{6022 \times 552}$ is constructed as explained 


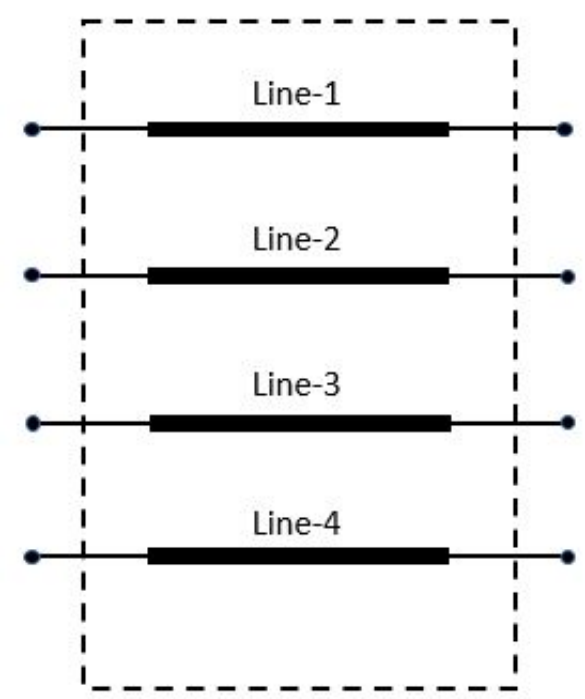

Figure 7.1: 4-Coupled transmission line network (example 1).

in Chapter 6 using five block-moments of frequency and two block-moments of each geometric parameter. Following a conventional PMOR approach [53], matching these block-moments of the original MTL structure leads to dense reduced system matrices $\hat{\mathbf{E}}_{i}$ of size $552 \times 552$ whose sparsity pattern is shown in the left panel of Fig. 7.2. The time required to build this reduced model is $6.19 \mathrm{~s}$. Next, following the steps of the proposed algorithm, through input clustering, e.g., one input in each cluster, the network is disjointed into eight decoupled subsystems. Each subsystem has an allocated input while the associated responses at all eight terminals are considered. Hence, each subsystem can be treated as a single-input and multi-output network in the reduction process. They share the same MNA matrices and sustain the dependency on all stochastic parameters. The projection matrix for each subsystems $\mathbf{V}_{k} \in \mathbb{R}^{6022 \times 69}$, for $k=1, \ldots, 8$ is constructed using five frequency and two parameter moments. Following this approach results in eight reduced-order models with system matrices $\hat{\mathbf{E}}_{k, i}(6.22)$ of size $69 \times 69$. Concatenating the resulting reduced subsystems, the overall reduced-order model with block diagonal-structured system matrices $\widehat{\boldsymbol{E}}_{i}$ (6.31) is constructed. The sparsity pattern for the system matrices of the resulting 
(overall) reduced model is shown in the right panel of Fig. 7.2. The time required to construct the reduced-order model for each subsystem is about $0.14 \mathrm{~s}$, leading to an overall reduced-model generation time of $1.16 \mathrm{~s}$.

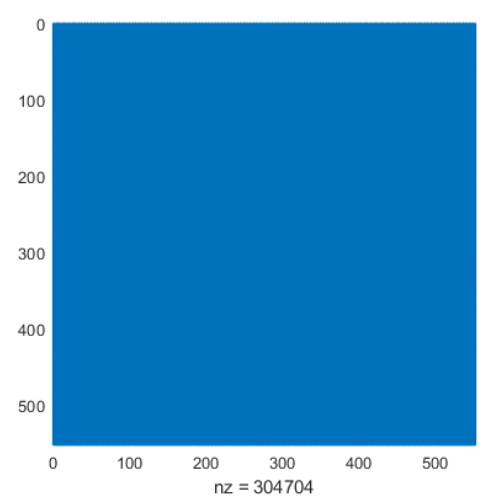

(a) PMOR

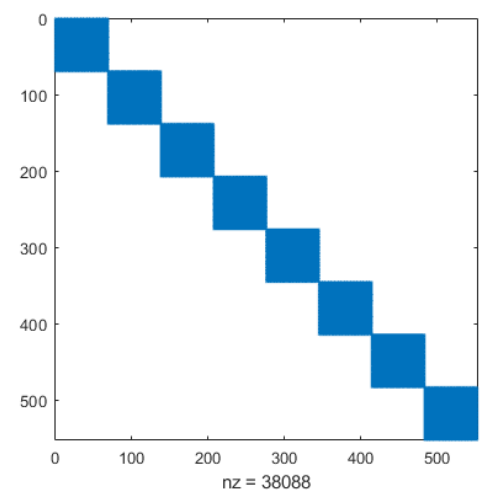

(b) Proposed

Figure 7.2: Sparsity pattern of reduced system matrices $\hat{\mathbf{E}}_{i}$ (left) and $\widehat{\boldsymbol{E}}_{i}$ (right) (example 1).

The three stochastic parameters with the given nominal values and standard deviations are taken into considerations. Based on 1000 Monte Carlo (MC) simulations, the statistical distributions of the outputs were obtained using the original model and the proposed and conventional PMOR methods. Each MC simulation features the mean and $\pm 3 \sigma$ deviation for 1000 distinct values of geometric parameters ranging between $\pm 10 \%$ from their nominal values. The variation of the responses at the ports $\# 2$ and \#4 are compared in Fig. 7.3 and Fig. 7.4, respectively. The results from the simulations of the original response (solid-line -), conventional PMOR $(+)$, and the proposed method $\left(^{*}\right)$ are signified in the graphs. As is seen, the results from the proposed approach are in good agreement with the results from the original and Conventional PMOR. 


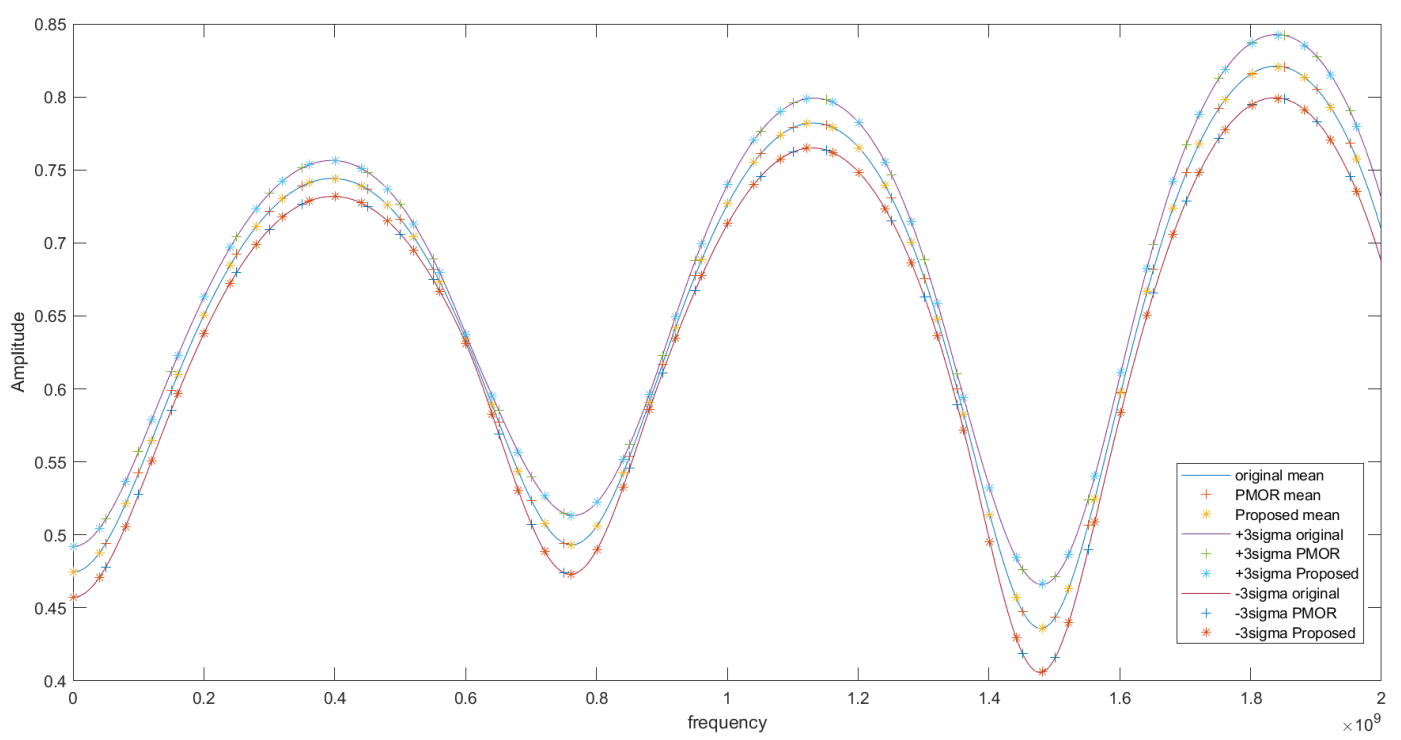

Figure 7.3: Statistical analysis for the response at the port \#2 (example 1).

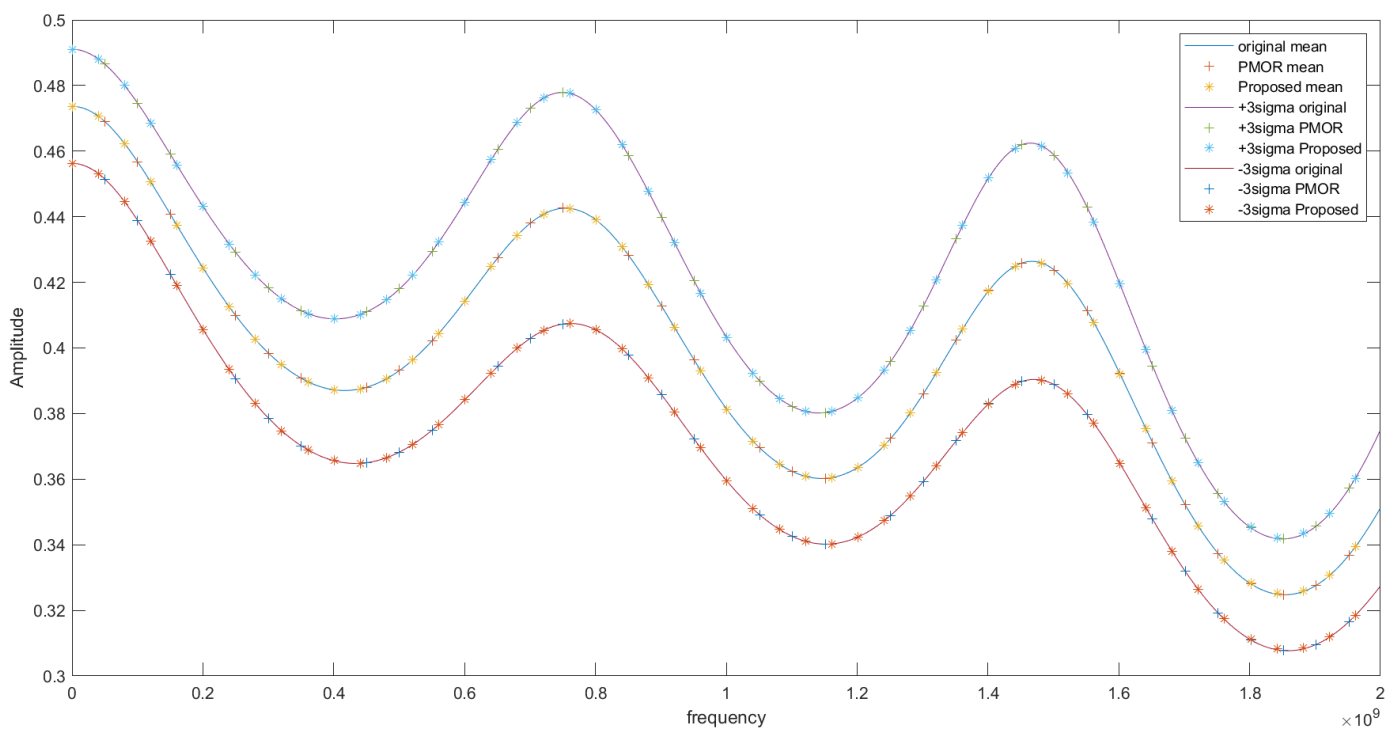

Figure 7.4: Statistical analysis for the response at the port \#4 (example 1).

Table 7.1 shows the comparison between the proposed method, conventional approach, and the original full model in terms of size,reduced-order model generation time, Monte Carlo simulation time, and speed-up. As seen, the proposed method yields a speedup of 87 versus the speedup of 10.5 attained from the conventional PMOR. 


\begin{tabular}{|c|c|c|c|c|}
\hline Model & Size & $\begin{array}{c}\text { Model Gen. } \\
\text { Time (s) }\end{array}$ & $\begin{array}{c}\text { MC Sim. } \\
\text { Time (s) }\end{array}$ & Speed-up \\
\hline Original & 6022 & - & 133,276 & - \\
\hline PMOR & 552 (Dense) & 6.19 & 12,657 & 10.5 \\
\hline Proposed & $\begin{array}{c}552 \\
\text { (Block Diagonal, Sparse) }\end{array}$ & 5.94 & 1,532 & 87 \\
\hline
\end{tabular}

Table 7.1: Size, model generation time, MC simulation time and speed-up comparisons (example 1).

For an eight-port interconnects structure in this example, the proposed PMOR algorithm demonstrates a significant advantage over conventional PMOR algorithms in computational cost. However, when the number of lines and the network's ports increases, the computational cost associated with the existing PMOR approaches is expected to rise rapidly, imposing serious efficiency challenges to the conventional PMOR methods. To comparatively demonstrate the performance and efficiency of the proposed method, a larger network with many ports is considered in the following example.

\subsection{Example 2}

In this example, the proposed method is examined using an interconnect structure with 24 coupled lines with 48 ports, as shown in Fig. 7.5. The over all structure of the system is same as previous example. Each line is $10 \mathrm{~cm}$ long. The model is generated using 250 section of lumped RLGC segmentation [83].

The four stochastic parameters are taken into considerations as the widths $(W)$ of the traces, spacing between the lines $(d)$, the conductivity of the material $(\sigma)$, and 


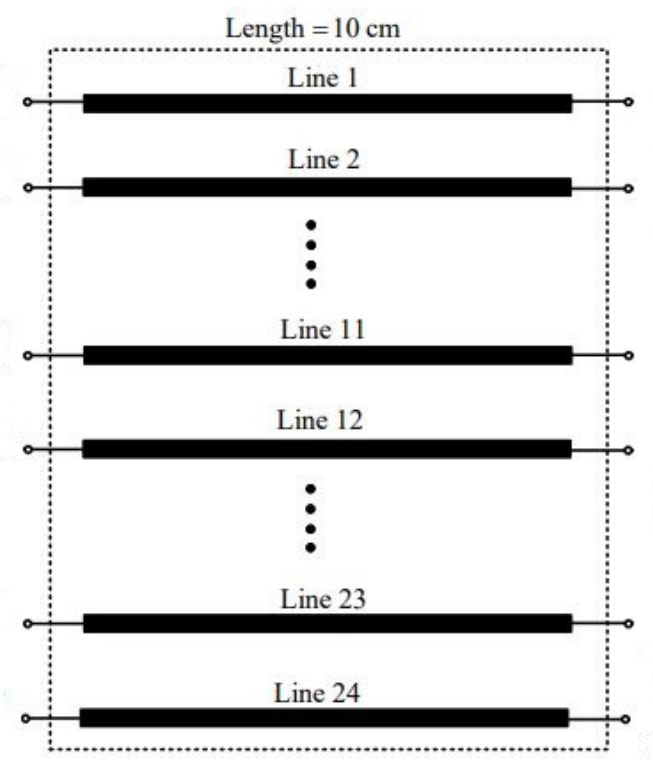

Figure 7.5: 24-Coupled transmission line network (example 2).

relative dielectric permittivity $\left(\epsilon_{r}\right)$. The nominal values of the stochastic physical parameters are $\mathrm{W}=9.2 \mathrm{~mm}, \mathrm{~d}=91 \mathrm{~mm}, \sigma=18.7 \times 10^{6} \mathrm{~S} / \mathrm{m}$, and $\epsilon_{r}=4.5$ and are assumed to have Gaussian distribution with a standard deviation of $\pm 10 \%$.

The number of the circuit variables is 36,122 . Thus, the size of the MNA matrices for the original network is $36,122 \times 36,122$. To reduce the parametrized network, the projection matrix $\mathbf{V} \in \mathbb{R}^{36,122 \times 3,888}$ is formed as explained in Chapter 4 to match fourblock moments of frequency and two block moments of each geometric parameter. The conventional PMOR method offers dense reduced system matrices $\hat{\mathbf{E}}_{i}$ of the size $3,888 \times 3,888$ when matching required block-moments of the original MTL. The sparse pattern of dense $\hat{\mathbf{E}}_{i}$ is shown in the left side of the Fig. 7.6. The CPU time required to construct this reduced model is $1380.8 \mathrm{~s}$. Now, for building the reduced model using proposed method, first we divide original system into 48 decoupled subsystems. Each subsystem has one input $\mathbf{B}_{i} \in \mathbb{R}^{36122 \times 1}$ and all 48 outputs $\mathbf{L} \in \mathbb{R}^{48 \times 36122}$. All the subsystems has the same MNA matrix and share same dependency on the design parameters. To reduce the subsystems a projection matrix $\mathbf{V}_{k} \in \mathbb{R}^{36122 \times 81}$ 
for $k=1, \ldots, 48$ is constructed as discussed in Chapter 6 using four frequency and two parameter moments. This gives 48 decoupled reduced subsystems whose system matrices $\hat{\mathbf{E}}_{k, i}$ (6.22) has the size of $81 \times 81$. Stacking the reduced subsystems give the over all reduced system whose system matrices $\widehat{\boldsymbol{E}}_{i}$ are sparse and block diagonal. The sparse pattern of this matrix is shown in the right side of Fig. 7.6. The system matrices are $98 \%$ sparse. To build one reduced subsystem required $1.13 \mathrm{~s}$ which means to form overall reduced system using proposed method takes $54.26 \mathrm{~s}$.

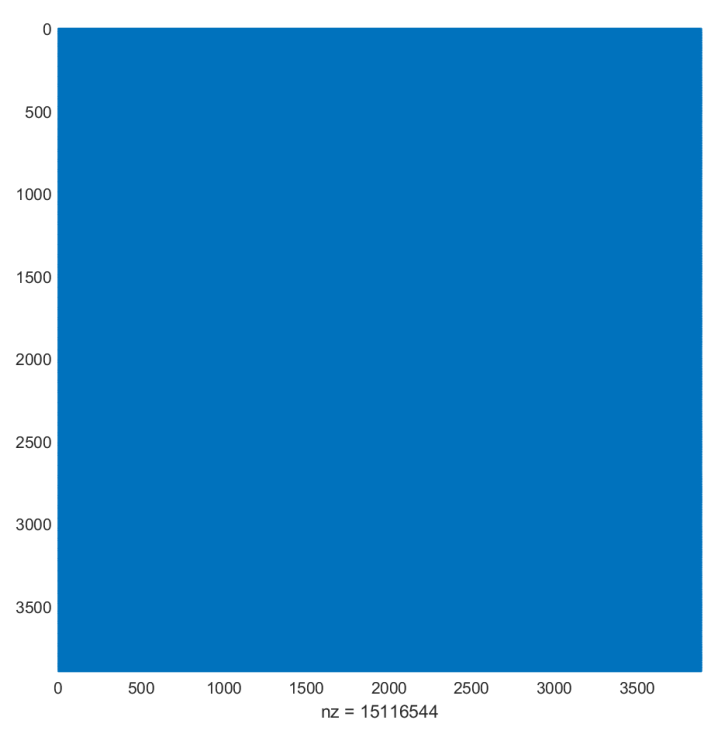

(a) PMOR

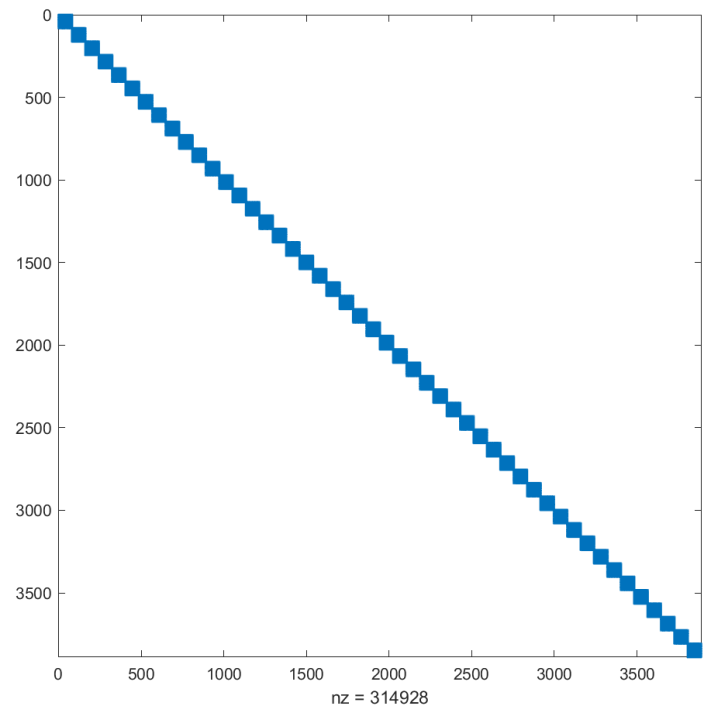

(b) Proposed

Figure 7.6: Sparsity pattern of reduced system matrices $\hat{\mathbf{E}}_{i}$ (left) and $\widehat{\boldsymbol{E}}_{i}$ (right) (example 2).

We have taken four stochastic physical parameters into consideration according to the nominal values and standard deviations. The statistical distributions of the outputs were obtained using both the proposed and conventional methods based on 500 Monte Carlo simulations, in which each simulation includes the mean and $\pm 3 \sigma$ deviation for 500 distinct values of geometric parameters ranging between $\pm 10 \%$ and their nominal values. The statistics of the responses at the port \#2 and \#48 computed using conventional and proposed methods are compared in Figures (7.7) and (7.8) 
to exhibit the accuracy of the proposed method. It is evident from the graphs that all these responses are in excellent agreement.

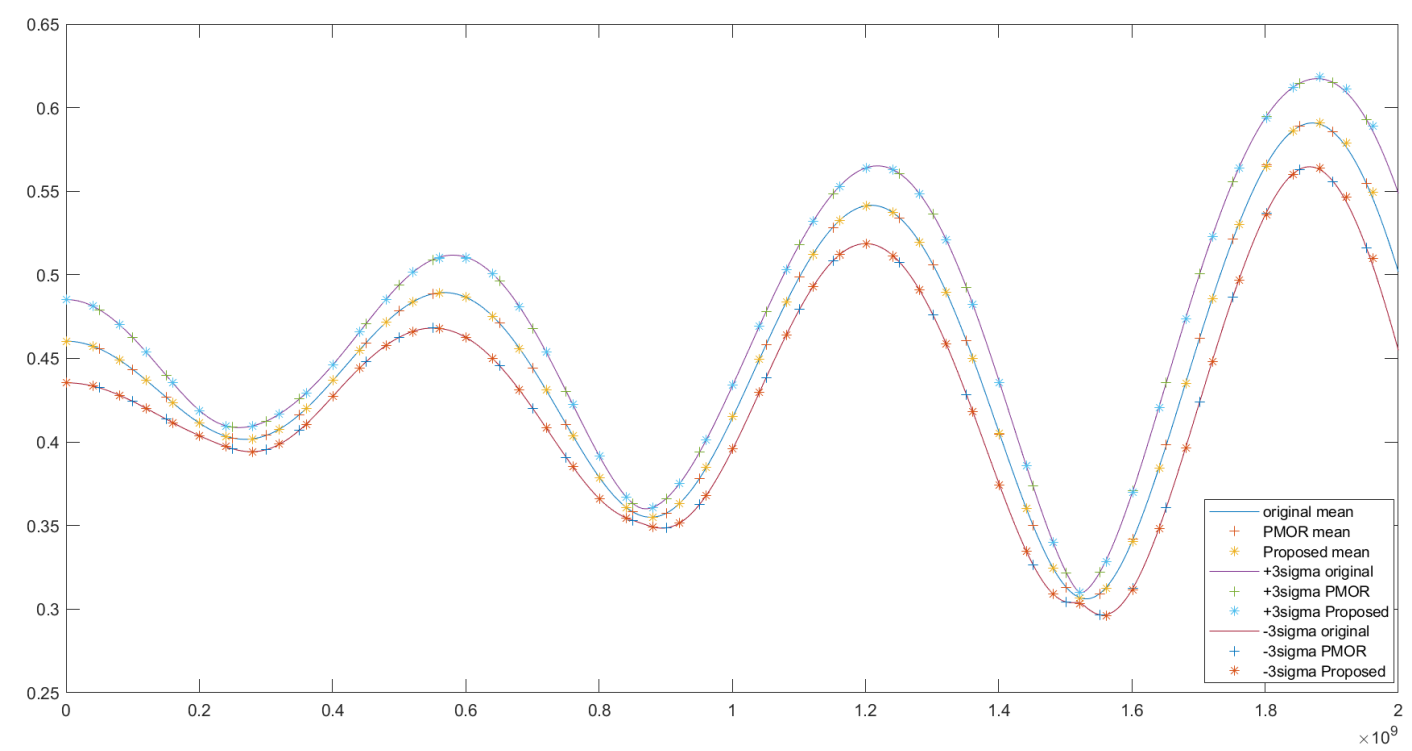

Figure 7.7: Statistical analysis for the response at port \#2. (example 2).

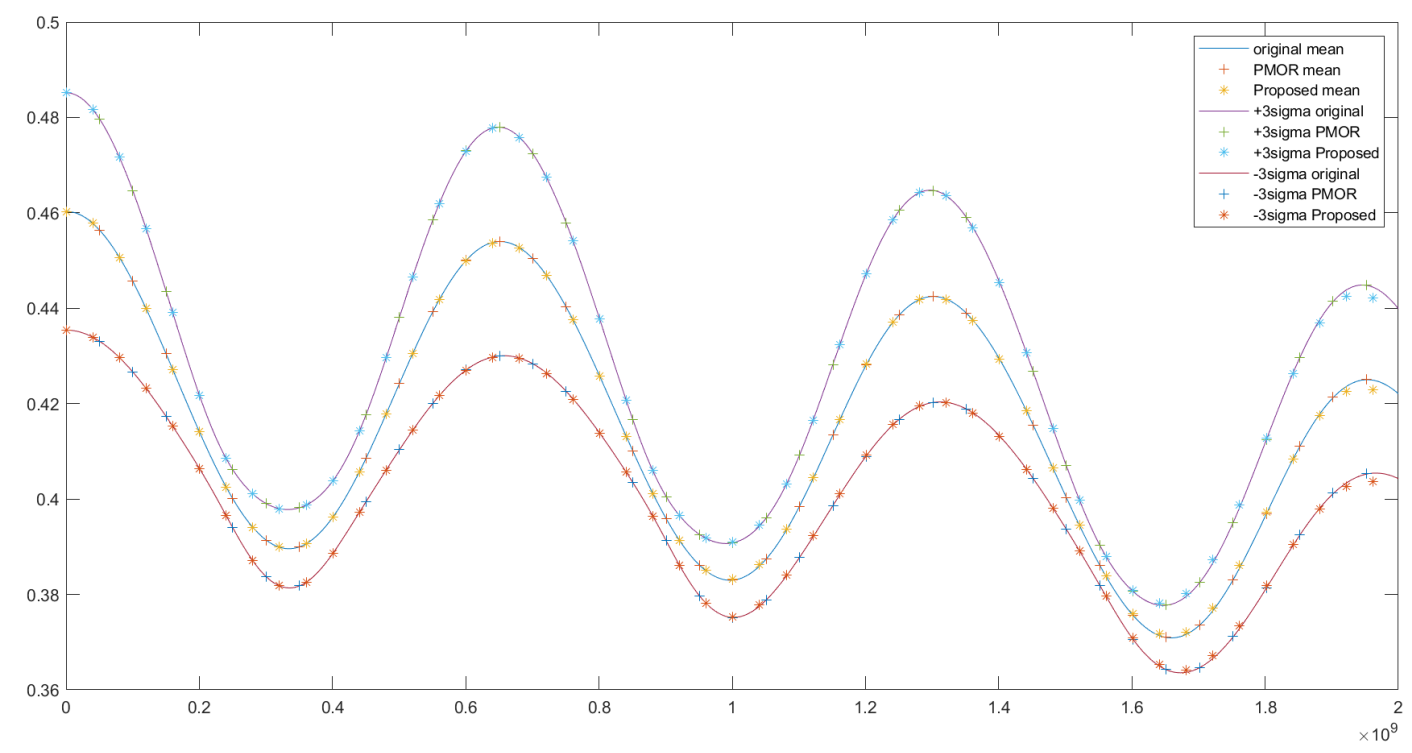

Figure 7.8: Statistical analysis for the response at port \#24. (example 2).

Table 7.2 compares the size and reduced-order model generation time, MC simulation time and speed-up of the original system versus the proposed and conventional 
PMOR. From the table, it is clear that the proposed method outperforms the conventional PMOR by providing a speedup of 194 times, while conventional PMOR can only achieve 1.7 times speedup compared to the direct simulation of the original circuits.

\begin{tabular}{|c|c|c|c|c|}
\hline Model & Size & $\begin{array}{c}\text { Model Gen. } \\
\text { Time (s) }\end{array}$ & $\begin{array}{c}\text { MC Sim. } \\
\text { Time (s) }\end{array}$ & Speed-up \\
\hline Original & 36122 & - & 670,283 & - \\
\hline PMOR & 3888 (Dense) & 1380.8 & 385,616 & 1.7 \\
\hline Proposed & $\begin{array}{c}3888 \\
\text { (Block Diagonal, Sparse) }\end{array}$ & 1362.6 & 3444 & 194 \\
\hline
\end{tabular}

Table 7.2: Size, model generation time, MC simulation time and speed-up comparisons (example 2). 


\section{Chapter 8}

\section{Conclusions and Future Work}

\subsection{Conclusions}

One of the major problem faced by circuit designers is rendering the behavior of designs in regards to their variations with respect to the key design parameters. An important example is the simulation of massively coupled multi-conductor interconnect networks when considering the geometric or environment parameters, which was the focus of this work.

This thesis introduced a new approach that brings the concept of PMOR to multiport circuits. The proposed method has been applied to obtain a reduced system of networks with a large number of ports that parameterizes the circuit response in several circuit design parameters.

The significant advantage of this approach is that it uses superposition, a fundamental paradigm for linear systems, to construct parametric reduced order models with sparse and block-diagonal system matrices. Hence, besides ensuring efficient simulations, the proposed method achieved accuracy by avoiding correlation assumptions or post-processing, causing accuracy degradation. Therefore, the proposed PMOR

method provides an edge in the statistical simulation of multi-parameter multi-port circuits. Numerical examples were presented showing the efficiency and accuracy of 
the proposed approach.

\subsection{Future Research}

Many practical circuits are stable but not passive. These circuits generally include dependent sources, e.g., small-signal device models and amplifier circuits. Using conventional MOR techniques (such as PRIMA) to reduce active circuits will not guarantee the stability of the resulting model. An efficient method has been proposed in [17] to preserve the stability of the (non-parametric) reduced-order model of active networks by construction. PMOR for large parametric active circuits is, in turn, arduous. Using the approach in [17], some reduction techniques have been developed for parametric active circuits in [84-86], mainly based on "Stochastic Collection" [87] and "Sparse Grid" [88, 89] techniques. However, PMOR of large multi-port active circuits with multiple stochastic parameters being tremendously challenging has remained an open issue. One of the main problems that will be the immediate focus in future research is "Stable model-order reduction of parameterized multi-port active networks." 


\section{List of References}

[1] W. H. A. Schilders, H. A. van der Vorstand, and J. Rommes, Eds., Model Order Reduction: Theory, Research Aspects and Applications. Berlin, Heidelberg, Germany: Springer-Verlag, 2008. 2, 12, 13, 16

[2] B. Nouri, M. Nakhla, and R. Achar, "A novel clustering scheme for reducedorder macromodeling of massively coupled interconnect structures," in Proc. IEEE 19th Conf. Electr. Perform. Electron. Packag.Syst, pp. 77-80, Oct. 2010. 2,44

[3] C. A. Desoer and E. S. Kuh, Basic Circuit Theory. New York, NY, USA: Prentice-Hall, 1969. 5

[4] C.-W. Ho, P. Brennan, and A. Ruehli, "The modified nodal approach to network analysis,," IEEE Transactions on Circuits and Systems, vol. 22, no. 6, p. 504-509, Jun 1975. 6

[5] J. Vlach and K. Singhal, Computer Methods for Circuit Analysis and Design,, 2nd ed. Boston, MA: Kluwer Academic Publishers, , 2003. 6

[6] F. N. Najm, Circuit Simulation. Hoboken, NJ: Wiley-IEEE Press,, 2010. 6, 39

[7] G. H. Golub and C. F. van Van Loan, Matrix Computations, 4th ed. Baltimore, MD, USA: Johns Hopkins Univ. Press, 2013. 10, 47, 62

[8] W. J. Rugh, Linear System Theory. USA: Prentice-Hall, Inc., 1996, vol. 2. 11

[9] U. Baur, P. Benner, and L. Feng, "Model order reduction for linear and nonlinear systems: A system-theoretic perspective," Arch. Computat. Methods Eng., vol. 21, no. 4, pp. 331-358, Dec. 2014. 12

[10] P. Benner, M. Hinz, and E. J. W. ter Maten, Eds., Model Reduction for Circuit Simulation. Berlin, Heidelberg, Germany: Springer-Verlag, 2011. 12 
[11] R. Pinnau, "Model reduction via proper orthogonal decomposition," in Model Order Reduction: Theory, Research Aspects and Applications, W. H. A. Schilders, H. A. van der Vorstand, and J. Rommes, Eds. Berlin, Heidelberg, Germany: Springer-Verlag, 2008, ch. 2, pp. 95-110. 12

[12] P. Astrid, S. Weiland, K. Willcox, and T. Backx, "Missing point estimation in models described by proper orthogonal decomposition," IEEE Trans. Autom. Control, vol. 53, no. 10, pp. 2237-2251, 2008. 12

[13] S. X. D. Tan and L. He, Advanced model order reduction techniques in VLSI design. Cambridge, MA, USA: Cambridge Univ. Press, 2007. 12, 13, 16, 39

[14] P. Benner, V. Mehrmann, and D. C. Sorensen, Eds., Dimension Reduction of Large-Scale Systems, ser. Lecture Notes in Computational Science and Engineering. Berlin, Heidelberg, Germany: Springer-Verlag, 2005, vol. 45. 12

[15] A. C. Antoulas, Approximation of large-scale dynamical systems. Philadelphia, PA, USA: SIAM, 2005. 12, 13, 15, 16

[16] B. Nouri, E. Gad, M. Nakhla, and R. Achar, "Model-order reduction in microelectronics," in Handbook on Model-Order Reduction: Theory, Research Aspects and Applications, ser. MORNET series, P. Benner, S. Grivet-Talocia, A. Quarteroni, G. Rozza, W. Schilders, and L. M. Silveira, Eds. Berlin, Germany: Walter de Gruyter GmbH, 2020, vol. 3, ch. 4, pp. 111-143. 12

[17] B. Nouri, M. S. Nakhla, and X. Deng, "Stable model-order reduction of active circuits," IEEE Trans. Compon., Packag., Manuf. Technol., vol. 7, no. 5, pp. 710-719, May 2017. 12, 74

[18] E. Gad, M. Nakhla, and R. Achar, "Model-order reduction of high-speed interconnects using integrated congruence transform," in Model Order Reduction: Theory, Research Aspects and Applications, ser. Mathematics in Industry, W. H. A. Schilders, H. A. van der Vorst, and J. Rommes, Eds. Berlin, Heidelberg, Germany: Springer-Verlag, 2008, vol. 13, ch. 17, pp. 361-401. 12

[19] M. J. Rewieński and J. White, "A trajectory piecewise-linear approach to model order reduction and fast simulation of nonlinear circuits and micromachined devices," IEEE Trans. Comput.-Aided Design Integr. Circuits Syst., vol. 22, no. 2, pp. 155-170, Feb. 2003. 12

[20] M. Celik, L. Pileggi, and A. Odabasioglu, IC interconnect analysis. Boston, MA, USA: Kluwer Academic, 2002. 12, 13, 16, 17, 18 
[21] A. Nouri, E. Gad, B. Nouri, and M. Nakhla, "Parameterized periodic steadystate analysis via reduced-order modelling," IEEE Trans. Microw. Theory Tech., pp. 1-12, Aug. 2021. 12

[22] B. Nouri and M. Nakhla, "Model-order reduction of nonlinear transmission lines using interpolatory proper orthogonal decomposition," IEEE Trans. Microw. Theory Tech., vol. 66, no. 12, pp. 5429-5438, Dec. 2018. 12

[23] B. Nouri, M. S. Nakhla, and R. Achar, "Efficient simulation of nonlinear transmission lines via model-order reduction," IEEE Trans. Microw. Theory Tech., vol. 65, no. 3, pp. 673-683, Mar. 2017. 12

[24] P. Li and L. T. Pileggi, "Compact reduced-order modeling of weakly nonlinear analog and RF circuits," IEEE Trans. Comput.-Aided Design Integr. Circuits Syst., vol. 24, no. 2, pp. 184-203, Feb 2005. 12

[25] E. Gad, R. Khazaka, R. Nakhla, and R. Griffith, "A circuit reduction technique for finding the steady-state solution of nonlinear circuits," IEEE Trans. Microw. Theory Tech., vol. 48, no. 12, pp. 2389-2396, 2000. 12

[26] B. Nouri, E. Gad, A. Nouri, and M. Nakhla, "DC-centric parameterized reducedorder model via moment-based interpolation projection (MIP) algorithm," IEEE Trans. Compon., Packag., Manuf. Technol., vol. 10, no. 8, pp. 1348-1357, Aug. 2020. 12

[27] P. Chen and C. Schwab, "Model order reduction methods in computational uncertainty quantification," in Handbook of Uncertainty Quantification, R. Ghanem, D. Higdon, and H. Owhadi, Eds. Basel, Switzerland: Springer Int. Publishing AG, 2017, ch. 27, pp. 937-990. 12

[28] D. Spina, F. Ferranti, G. Antonini, T. Dhaene, and L. Knockaert, "Efficient variability analysis of electromagnetic systems via polynomial chaos and model order reduction," IEEE Trans. Compon., Packag., Manuf. Technol., vol. 4, no. 6, pp. 1038-1051, Jun. 2014. 12

[29] Y. Tao, B. Nouri, M. S. Nakhla, M. Farhan, and R. Achar, "Variability analysis via parameterized model order reduction and numerical inversion of Laplace transform," IEEE Trans. Compon., Packag., Manuf. Technol., vol. 7, no. 5, pp. 678-686, Jan. 2017. 12, 21, 36

[30] L. Lombardi, Y. Tao, B. Nouri, M. Nakhla, F. Ferranti, and G. Antonini, "Parameterized model order reduction of delayed PEEC circuits," IEEE Trans. Electromagn. Compat., vol. 62, no. 3, pp. 859-869, Jun. 2020. 12, 21 
[31] D. Szypulski, G. Fotyga, V. de la Rubia, and M. Mrozowski, "A subspacesplitting moment-matching model-order reduction technique for fast wideband fem simulations of microwave structures," IEEE Trans. Microw. Theory Tech., vol. 68, no. 8, pp. 3229-3241, 2020. 12

[32] L. Feng and P. Benner, "Parametric model order reduction for electro-thermal coupled problems," in Nanoelectronic Coupled Problems Solutions. Springer, 2019, pp. 293-309. 12

[33] G. Thrivikraman, S. L. Johnson, Z. H. Syedain, R. C. Hill, K. C. Hansen, H. S. Lee, and R. T. Tranquillo, "Biologically-engineered mechanical model of a calcified artery," Acta biomaterialia, vol. 110, pp. 164-174, 2020. 12

[34] M. Xiao, D. Lu, P. Breitkopf, B. Raghavan, S. Dutta, and W. Zhang, "On-the-fly model reduction for large-scale structural topology optimization using principal components analysis," Structural and Multidisciplinary Optimization, pp. 1-22, 2020. 12

[35] S. Ponsioen, S. Jain, and G. Haller, "Model reduction to spectral submanifolds and forced-response calculation in high-dimensional mechanical systems," Journal of Sound and Vibration, vol. 488, p. 115640, 2020. 12

[36] P. Benner, S. Grivet-Talocia, A. Quarteroni, G. Rozza, W. Schilders, and L. Silveira, Eds., Model Order Reduction. Volume 1: System- and Data-Driven Methods and Algorithms. Berlin: De Gruyter, 2020. 13

[37] — , Model Order Reduction. Volume 2: Snapshot-Based Methods and Algorithms. Berlin: De Gruyter, 2020. 13

[38] —, Model Order Reduction. Volume 3: Applications. Berlin: De Gruyter, 2020. 13

[39] Y. Saad, Iterative Methods for Sparse Linear Systems. Philadelphia.: Society for Industrial Applied Mathematics,, 2003. 15

[40] P. Feldmann and R. W. Freund, "Efficient linear circuit analysis by Padé approximation via the Lanczos process," IEEE Trans. Comput.-Aided Design Integr. Circuits Syst., vol. 14, no. 5, pp. 639-649, May 1995. 16

[41] R. W. Freund, "Krylov-subspace methods for reduced-order modeling in circuit simulation," Comput. Appl. Math., vol. 123, no. 1-2, pp. 395-421, Nov. 2000. 16, 39 
[42] A. Odabasioglu, M. Celik, and L. Pileggi, "PRIMA: passive reduced-order interconnect macromodeling algorithm," vol. 17, no. 8, Aug. 1998, pp. 645-654. 16

[43] E. J. Grimme, "Krylov projection methods for model reduction," Ph.D. dissertation, Dept. Elect. Comput. Eng., Univ. Illinois Urbana-Champaign, Champaign, IL, USA, 1997. 16, 31

[44] C. de Villemagne and R. E. Skelton, "Model reductions using a projection formulation," in Proc. 26th IEEE Conference on Decision and Control, Los Angeles, CA, USA, 1987, pp. 461-466. 16

[45] P. Benner, S. Gugercin, and K. Willcox, "A survey of projection-based model reduction methods for parametric dynamical systems," SIAM Review, vol. 57, no. 4, pp. 483-531, Nov. 2015. 16, 21, 28

[46] W. E. Arnoldi, "The principle of minimized iteration in the solution of the matrix eigenvalue problem," Quat. Appl. Math., vol. 9, no. 1, pp. 17-29, Apr. 1951. 18

[47] R. Achar and M. Nakhla, "Simulation of high-speed interconnects," Proceedings of the IEEE, vol. 89, no. 5, pp. 719-724, 2001. 18

[48] A. Odabasioglu, M. Celik, and L. Pileggi, "PRIMA: passive reduced-order interconnect macromodeling algorithm," IEEE Transactions on Computer-Aided Design of Integrated Circuits and Systems, vol. 17, no. 8, pp. 645-654, Aug. 1998. $19,39,43$

[49] X. Deng, B. Nouri, and M. Nakhla, "Stable model-order reduction of active circuits," IEEE Transactions on Components, Packaging and Manufacturing Technology, vol. PP, pp. 1-10, 01 2017. 20

[50] A. Quarteroni, A. Manzoni, and F. Negri, Reduced basis methods for partial differential equations: an introduction, ser. MS\&A - Modeling, Simulation and Applications. New York, NY, USA: Springer, 2016, vol. 92. 21, 28

[51] J. S. Hesthaven, G. Rozza, and B. Stamm, Certified Reduced Basis Methods for Parametrized Partial Differential Equations, ser. SpringerBriefs in Mathematics. Basel, Switzerland: Springer Int. Publishing AG, 2016. 21

[52] P. Benner, M. Ohlberger, A. Patera, G. Rozza, and K. Urban, Eds., Model reduction of parametrized systems, ser. Modeling, Simulation and Applications (MS\&A). Basel, Switzerland: Springer Int. Publishing AG, 2017, vol. 17. 21, 28 
[53] P. Benner and L. Feng, "A robust algorithm for parametric model order reduction based on implicit moment matching," in Reduced order methods for modeling and computational reduction, ser. MS\&A Modeling, Simulation and Applications, A. Quarteroni and G. Rozza, Eds. Berlin, Heidelberg, Germany: SpringerVerlag, 2014, vol. 9, ch. 6, pp. 159-185. 21, 28, 31, 55, 58, 60, 65

[54] L. Daniel, O. C. Siong, L. S. Chay, K. H. Lee, and J. White, "A multiparameter moment-matching model-reduction approach for generating geometrically parameterized interconnect performance models," IEEE Trans. Comput.-Aided Design Integr. Circuits Syst., vol. 23, no. 5, pp. 678-693, May 2004. 21, 28, 29, $36,58,60,84,87$

[55] X. Li, P. Li, and L. T. Pileggi, "Parameterized interconnect order reduction with explicit-and-implicit multi-parameter moment matching for inter/intra-die variations," in Proc. ICCAD-2005 Computer-Aided Design IEEE/ACM International Conference on, Nov. 6-10, 2005, pp. 806-812. 21, 28

[56] Y.-T. Li, Z. Bai, Y. Su, and X. Zeng, "Model order reduction of parameterized interconnect networks via a two-directional arnoldi process," IEEE Trans. Comput.-Aided Design Integr. Circuits Syst., vol. 27, no. 9, pp. 1571-1582, Sep. 2008. 21,28

[57] Y. Liu, L. Pileggi, and A. Strojwas, "Model order-reduction of RC(L) interconnect including variational analysis," in Proc. 36th IEEE/AMC Des. Autom. Conf., 1999, pp. 201-206. 21

[58] F. Ferranti, G. Antonini, T. Dhaene, and L. Knockaert, "Guaranteed passive parameterized model order reduction of the partial element equivalent circuit (PEEC) method," IEEE Trans. Electromagn. Compat., vol. 52, no. 4, pp. 974984, Nov. 2010. 21

[59] F. Ferranti, G. Antonini, T. Dhaene, L. Knockaert, and A. E. Ruehli, "Physicsbased passivity-preserving parameterized model order reduction for PEEC circuit analysis," IEEE Trans. Compon., Packag., Manuf. Technol., vol. 1, no. 3, pp. 399-409, Mar. 2011. 21

[60] R. Achar and M. S. Nakhla, "Simulation of high-speed interconnects," Proceedings of the IEEE, vol. 89, no. 5, pp. 693-728, 2001. 38

[61] A. Dounavis, X. Li, R. Nakhla, and R. Achar, "Passive closed-form transmissionmodel for general purpose circuit simulators," IEEE Trans. Microw. Theory Tech, vol. 47, no. 12, p. 2450-2459, 1999. 38 
[62] A. Dounavis, R. Achar, and M. Nakhla, "Efficient passive circuit models for distributed networks with frequency-dependent parameters," IEEE Transactions on Advanced Packaging, vol. 23, no. 3, pp. 382-392, 2000. 38

[63] E. Gad, A. Dounavis, M. Nakhla, and R. Achar, "Passive model order reduction of multiport distributed interconnects," "IEEE Transactions on Microwave Theory and Techniques, vol. 48, no. 12, p. 2325-2334, 2000. 38

[64] A. Dounavis, R. Achar, and M. Nakhla, "A general class of passive macromodels for lossy multiconductor transmission lines," IEEE Transactions on Microwave Theory and Techniques, vol. 49, no. 10, pp. 1686-1696, 2001. 38

[65] H. Yu, C. Chu, Y. Shi, D. Smart, L. He, and S. X.-D. Tan, "Fast analysis of a large-scale inductive interconnect by block-structure-preserved macromodeling," IEEE Trans. Very Large Scale Integr. Syst., vol. 18, no. 10, p. 1399-1411, Oct. 2010. 38

[66] N. Nakhla, A. Ruehli, M. Nakhla, and R. Achar, "Simulation of coupled interconnects using waveform relaxation and transverse partitioning," Advanced Packaging, IEEE Transactions on, pp. 78- 87, Feb 2006. 38

[67] N. Nakhla, A. Ruehli, M. Nakhla, R. Achar, and C. Chen, "Waveform relaxation techniques for simulation of coupled interconnects with frequency-dependent parameters," Advanced Packaging, IEEE Transactions on, vol. 30, pp. 257 - 269, May 2007. 38

[68] M. Farhan, N. Nakhla, M. Nakhla, R. Achar, and A. Ruehli, "Overlapping partitioning techniques for simulation of strongly coupled distributed interconnects," IEEE Transactions on Components, Packaging and Manufacturing Technology, vol. 2, pp. 1193-1201, 2012. 38

[69] P. Feldmann and R. W. Freund, "Efficient linear circuit analysis by padeápproximation via the lanczos process," IEEE Transactions on ComputerAided Design of Integrated Circuits and Systems, vol. 14, no. 5, pp. 639-649, May 1995. 39

[70] Z. Bai, "Krylov subspace techniques for reduced-order modeling of large-scale dynamical systems,", Applied numerical mathematics, vol. 43, no. 1, pp. 9-44, Oct 2002. 39

[71] R. W. Freund and P. Feldmann, "The sympvl algorithm and its applications to interconnect simulation," in International Conference on Simulation of Semiconductor Processes and Devices, Sep. 1997, pp. 113-116. 
[72] R. Freund, "SPRIM: structure-preserving reduced-order interconnect macromodeling," in IEEE/ACM International Conference on Computer-Aided Design, Nov. 2004, pp. 80-87. 39

[73] L. Miguel Silveira, M. Kamon, I. Elfadel, and J. White, "A coordinatetransformed arnoldi algorithm for generating guaranteed stable reduced-order models of RLC circuits," in IEEE/ACM International Conference on ComputerAided Design, Nov. 1996, pp. 288 -294. 39

[74] L. Miguel Silveira, M. Kamon, and J. White, "Efficient reduced-order modeling of frequency-dependent coupling inductances associated with 3-D interconnect structures," IEEE Transactions on Components, Packaging, and Manufacturing Technology, Part B: Advanced Packaging, vol. 19, no. 2, pp. 283-288, May 1996. 39

[75] P. Feldmann, "Model order reduction techniques for linear systems with large numbers of terminals," Proc. Design, Autom. Test Eur. Conf. Exhibit, vol. 2, pp. 944-947, Feb 2004. 39

[76] C.-T. Chen, Linear System Theory and Design. New York: Oxford Univ. Press, 1998. 39

[77] P. Feldmann and F. Liu, "Sparse and efficient reduced order modeling of linear subcircuits with large number of terminals," in IEEE/ACM International Conference on Computer Aided Design, 2004. ICCAD-2004., Nov. 2004, pp. 88-92. 41

[78] P. Liu, S.-D. Tan, B. Yan, and B. McGaughy, "An extended svd-based terminal and model order reduction algorithm," in Proc. 2006 IEEE Int.Behav. Model. Simulat. Workshop, pp. 44-49, Sep 2006. 41

[79] P. Liu, S.-D. Tan, H. Li, Z. Qi, J. Kong, B. McGaughy, and L. He, "An efficient method for terminal reduction of interconnect circuits considering delay variations," in Proc. IEEE/ACM Int. Conf. Comput.- Aided Design, pp. 821-826, Nov 2005. 41

[80] P. Liu, S.-D. Tan, B. McGaughy, and L. Wu, "Compact reduced order modeling for multiple-port interconnects," in Proc. 7th Int. Symp. Quality Electron. Design, pp. 418-424, Mar 2006. 41 
[81] P. Liu, S.-D. Tan, B. McGaughy, L. Wu, and L. He, "Termmerg: An efficient terminal-reduction method for interconnect circuits," IEEE Trans. Comput.Aided Design Integr. Circuits Syst., vol. 26, no. 8, pp. 1382-1392, Aug 2007. 41

[82] B. D. O. Anderson and S. Vongpanitlerd, Network Analysis and Synthesis. Englewood Cliffs, NJ: Prentice Hall, 1973. 47, 62

[83] C. Paul, Analysis of Multiconductor Transmission Lines. New York, NY:Wiley, 1994, vol. 6. 64, 68

[84] Y. Tao, B. Nouri, F. Ferranti, M. Nakhla, and K. Guo, "Non-intrusive variability analysis of large circuits with parallelism in the stochastic space and timedomain," in 2020 International Symposium on Electromagnetic Compatibility, EMC, EUROPE, Sep. 2020, pp. 1-5. 74

[85] K. Guo, F. A. Sheikh, B. Nouri, F. Ferranti, and M. Nakhla, "Efficient timedomain variability analysis of active circuits," in Proc. IEEE Elect. Des. Adv. Packag. and Syst. Symp., Honolulu, HI, USA, Dec. 2016, pp. 1-4. 74

[86] K. Guo, F. Ferranti, B. Nouri, and M. Nakhla, "A stochastic collocation technique for time-domain variability analysis of active circuits," in Proc. 25th IEEE Conf. Elect. Perform. Electron. Packag. Syst., San Diego, CA, USA, Oct. 2016, pp. 47-50.

[87] L. W.-T. Ng and M. S. Eldred, "Multifidelity uncertainty quantification using non-intrusive polynomial chaos and stochastic collocation," in Proc. AIAA Structures, Structural Dynamics and Materials Conference, vol. 43, no. AIAA20121852, Honolulu, HI, USA, Apr. 2012, pp. 1-17. 74

[88] F. Nobile, R. Tempone, and C. G. Webster, "A sparse grid stochastic collocation method for partial differential equations with random input data," SIAM J. Numer. Anal., vol. 46, no. 5, pp. 2309-2345, Sep. 2008. 74

[89] N. Agarwal and N. R. Aluru, "Weighted smolyak algorithm for solution of stochastic differential equations on non-uniform probability measures," Int $J$ Numer Meth Eng, vol. 85, no. 11, pp. 1365-1389, Mar. 2011. 74

[90] B. Nouri, M. S. Nakhla, and R. Achar, "Efficient reduced-order macromodels of massively coupled interconnect structures via clustering," IEEE Trans. Compon., Packag., Manuf. Technol., vol. 3, no. 5, pp. 826-840, May 2013. 88, 94 


\section{Appendix A}

\section{Mathematical Proof of Lemma 3}

Following the steps of the proof presented in [54] to prove Lemma 3, we need the technique called Mathematical Induction. Let's start with $m=0$ :

$$
\begin{array}{r}
\hat{\mathbf{F}}_{k_{2}, \ldots, k_{p}}^{0}\left[-\left(\mathbf{V}^{T} \tilde{\mathbf{E}}_{0} \mathbf{V}\right)^{-1} \mathbf{V}^{T} \tilde{\mathbf{E}}_{1} \mathbf{V}, \ldots,-\left(\mathbf{V}^{T} \tilde{\mathbf{E}}_{0} \mathbf{V}\right)^{-1} \mathbf{V}^{T} \tilde{\mathbf{E}}_{p} \mathbf{V}\right] \\
\left(\mathbf{V}^{T} \tilde{\mathbf{E}}_{0} \mathbf{V}\right)^{-1} \mathbf{V}^{T} \mathbf{B} \\
=\mathbf{I}\left(\mathbf{V}^{T} \tilde{\mathbf{E}}_{0} \mathbf{V}\right)^{-1} \mathbf{V}^{T} \mathbf{B}
\end{array}
$$

$\tilde{\mathbf{E}}_{0}^{-1} \mathbf{B} \in \operatorname{colspan}(\mathbf{V})$, from Lemma 2 we have

$$
\begin{aligned}
\left(\mathbf{V}^{T} \tilde{\mathbf{E}}_{0} \mathbf{V}\right)^{-1} \mathbf{V}^{T} \mathbf{B} & =\mathbf{V}^{T} \tilde{\mathbf{E}}_{0}^{-1} \mathbf{B} \\
& =\mathbf{V}^{T} \mathbf{F}_{k_{2}, \ldots, k_{p}}^{0}\left[-\tilde{\mathbf{E}}_{0}^{-1} \tilde{\mathbf{E}}_{1}, \ldots,-\tilde{\mathbf{E}}_{0}^{-1} \tilde{\mathbf{E}}_{1}\right] \tilde{\mathbf{E}}_{0}^{-1} \mathbf{B}
\end{aligned}
$$

So, the above equation prove that Lemma 3 hold true for $m=0$. As per mathematical induction, if statement hold true for order m-1 then it is also correct for order $\mathrm{m}$.

$$
\begin{aligned}
& \hat{\mathbf{F}}_{k_{2}, \ldots, k_{p}}^{m}\left[-\left(\mathbf{V}^{T} \tilde{\mathbf{E}}_{0} \mathbf{V}\right)^{-1} \mathbf{V}^{T} \tilde{\mathbf{E}}_{1} \mathbf{V}, \ldots,-\left(\mathbf{V}^{T} \tilde{\mathbf{E}}_{0} \mathbf{V}\right)^{-1} \mathbf{V}^{T} \tilde{\mathbf{E}}_{p} \mathbf{V}\right]\left(\mathbf{V}^{T} \tilde{\mathbf{E}}_{0} \mathbf{V}\right)^{-1} \mathbf{V}^{T} \mathbf{B}= \\
= & {\left[\left(\mathbf{V}^{T} \tilde{\mathbf{E}}_{0} \mathbf{V}\right)^{-1} \mathbf{V}^{T} \tilde{\mathbf{E}}_{1} \mathbf{V} \hat{\mathbf{F}}_{k_{2}, \ldots, k_{p}}^{m-1}\left(-\left(\mathbf{V}^{T} \tilde{\mathbf{E}}_{0} \mathbf{V}\right)^{-1} \mathbf{V}^{T} \tilde{\mathbf{E}}_{1} \mathbf{V}, \ldots,-\left(\mathbf{V}^{T} \tilde{\mathbf{E}}_{0} \mathbf{V}\right)^{-1} \mathbf{V}^{T} \tilde{\mathbf{E}}_{p} \mathbf{V}\right) \ldots\right.}
\end{aligned}
$$




$$
\begin{gathered}
\ldots+\left(\mathbf{V}^{T} \tilde{\mathbf{E}}_{0} \mathbf{V}\right)^{-1} \mathbf{V}^{T} \tilde{\mathbf{E}}_{1} \mathbf{V} \hat{\mathbf{F}}_{k_{2}, \ldots, k_{p}-1}^{m-1}\left(-\left(\mathbf{V}^{T} \tilde{\mathbf{E}}_{0} \mathbf{V}\right)^{-1} \mathbf{V}^{T} \tilde{\mathbf{E}}_{1} \mathbf{V}, \ldots,\right. \\
\left.\left.\ldots,\left(\mathbf{V}^{T} \tilde{\mathbf{E}}_{0} \mathbf{V}\right)^{-1} \mathbf{V}^{T} \tilde{\mathbf{E}}_{p} \mathbf{V}\right)\right]\left(\mathbf{V}^{T} \tilde{\mathbf{E}}_{0} \mathbf{V}\right)^{-1} \mathbf{V}^{T} \mathbf{B} \\
=\left[\left(\mathbf{V}^{T} \tilde{\mathbf{E}}_{0} \mathbf{V}\right)^{-1} \mathbf{V}^{T} \tilde{\mathbf{E}}_{1} \mathbf{V} \mathbf{V}^{T} \hat{\mathbf{F}}_{k_{2}, \ldots, k_{p}}^{m-1}\left(-\tilde{\mathbf{E}}_{0}^{-1} \tilde{\mathbf{E}}_{1}, \ldots, \tilde{\mathbf{E}}_{0}^{-1} \tilde{\mathbf{E}}_{p}\right)+\ldots\right. \\
\left.\ldots+\left(\mathbf{V}^{T} \tilde{\mathbf{E}}_{0} \mathbf{V}\right)^{-1} \mathbf{V}^{T} \tilde{\mathbf{E}}_{1} \mathbf{V} \mathbf{V}^{T} \hat{\mathbf{F}}_{k_{2}, \ldots, k_{p}-1}^{m-1}\left(-\tilde{\mathbf{E}}_{0}^{-1} \tilde{\mathbf{E}}_{1}, \ldots,-\tilde{\mathbf{E}}_{0}^{-1} \tilde{\mathbf{E}}_{p}\right)\right] \tilde{\mathbf{E}}_{0}^{-1} \mathbf{B} \\
=\left[\left(\mathbf{V}^{T} \tilde{\mathbf{E}}_{0} \mathbf{V}\right)^{-1} \mathbf{V}^{T} \tilde{\mathbf{E}}_{1} \hat{\mathbf{F}}_{k_{2}, \ldots, k_{p}}^{m-1}\left(-\tilde{\mathbf{E}}_{0}^{-1} \tilde{\mathbf{E}}_{1}, \ldots, \tilde{\mathbf{E}}_{0}^{-1} \tilde{\mathbf{E}}_{p}\right)+\ldots\right. \\
\left.\ldots+\left(\mathbf{V}^{T} \tilde{\mathbf{E}}_{0} \mathbf{V}\right)^{-1} \mathbf{V}^{T} \tilde{\mathbf{E}}_{1} \hat{\mathbf{F}}_{k_{2}, \ldots, k_{p}-1}^{m-1}\left(-\tilde{\mathbf{E}}_{0}^{-1} \tilde{\mathbf{E}}_{1}, \ldots,-\tilde{\mathbf{E}}_{0}^{-1} \tilde{\mathbf{E}}_{p}\right)\right] \tilde{\mathbf{E}}_{0}^{-1} \mathbf{B} \\
=\left(\mathbf{V}^{T} \tilde{\mathbf{E}}_{0} \mathbf{V}\right)^{-1} \mathbf{V}^{T}\left[\tilde{\mathbf{E}}_{1} \hat{\mathbf{F}}_{k_{2}, \ldots, k_{p}}^{m-1}\left(-\tilde{\mathbf{E}}_{0}^{-1} \tilde{\mathbf{E}}_{1}, \ldots, \tilde{\mathbf{E}}_{0}^{-1} \tilde{\mathbf{E}}_{p}\right)+\ldots\right. \\
\left.\ldots+\tilde{\mathbf{E}}_{1} \hat{\mathbf{F}}_{k_{2}, \ldots, k_{p}-1}^{m-1}\left(-\tilde{\mathbf{E}}_{0}^{-1} \tilde{\mathbf{E}}_{1}, \ldots,-\tilde{\mathbf{E}}_{0}^{-1} \tilde{\mathbf{E}}_{p}\right)\right] \tilde{\mathbf{E}}_{0}^{-1} \mathbf{B}
\end{gathered}
$$

use Lemma 1 and Lemma 2 on all the term of the equation A.4 and obtained the equation A.5.

$$
=\mathbf{V}^{T} \hat{\mathbf{F}}_{k_{2}, \ldots, k_{p}}^{m}\left(-\tilde{\mathbf{E}}_{0}^{-1} \tilde{\mathbf{E}}_{1}, \ldots, \tilde{\mathbf{E}}_{0}^{-1} \tilde{\mathbf{E}}_{p}\right) \tilde{\mathbf{E}}_{0}^{-1} \mathbf{B}
$$




\section{Appendix B}

\section{Mathematical Proof of Theorem 2}

The system represent in equation 4.9 for a single-input case where $\mathbf{B}=\mathbf{b} \in \mathbb{R}^{n \times 1}$ is given as:

$$
\begin{gathered}
\mathbf{H}=\mathbf{L}\left[\mathbf{I}-\left(\tilde{\lambda}_{1} \mathbf{M}_{1}+\cdots+\tilde{\lambda}_{p} \mathbf{M}_{p}\right)^{-1}\right] \tilde{\mathbf{E}}_{0} \mathbf{b} \\
\mathbf{H}=\sum_{m=0}^{\infty} \sum_{k_{2}=0}^{m-\left(k_{3}+\cdots+k_{p}\right)} \cdots \sum_{k_{p}-1=0}^{m-k_{p}} \sum_{k_{p}}^{m}\left\{\left[\mathbf{L} \mathbf{F}_{k_{2}, \ldots, k_{p}}^{m}\left(\mathbf{M}_{1}, \ldots, \mathbf{M}_{p}\right) \mathbf{b}_{M}\right]\right\} \ldots \\
\ldots \tilde{\lambda}_{1}^{m-\left(k_{2}+\cdots+k_{p}\right)} \tilde{\lambda}_{2}^{k_{2}} \ldots \tilde{\lambda}_{p}^{k_{p}}
\end{gathered}
$$

same way the system showed in equation 4.21 can be write as:

$$
\begin{gathered}
\hat{\mathbf{H}}=\mathbf{L V}\left\{\mathbf{I}-\left[\tilde{\lambda}_{1}\left(\mathbf{V}^{T}\left(-\tilde{\mathbf{E}}_{0}\right) \mathbf{V}\right)^{-1} \mathbf{V}^{T} \tilde{\mathbf{E}}_{1} \mathbf{V}+\ldots\right.\right. \\
\left.\left.\ldots+\tilde{\lambda}_{p}\left(\mathbf{V}^{T}\left(-\tilde{\mathbf{E}}_{0}\right) \mathbf{V}\right)^{-1} \mathbf{V}^{T} \tilde{\mathbf{E}}_{p} \mathbf{V}\right]\right\}^{-1}\left(\mathbf{V}^{T} \tilde{\mathbf{E}}_{0} \mathbf{V}\right)^{-1} \mathbf{V}^{T} \mathbf{b} \\
=\sum_{m=0}^{\infty} \sum_{k_{2}=0}^{m-\left(k_{3}+\cdots+k_{p}\right)} \cdots \sum_{k_{p}-1=0}^{m-k_{p}} \sum_{k_{p}}^{m}\left\{\left[\mathbf { L V } \hat { \mathbf { F } } _ { k _ { 2 } , \ldots , k _ { p } } ^ { m } \left(-\left(\mathbf{V}^{T} \tilde{\mathbf{E}}_{0} \mathbf{V}\right)^{-1} \mathbf{V}^{T} \tilde{\mathbf{E}}_{1} \mathbf{V} \ldots\right.\right.\right.
\end{gathered}
$$




$$
\left.\left.\left.\cdots-\left(\mathbf{V}^{T} \tilde{\mathbf{E}}_{0} \mathbf{V}\right)^{-1} \mathbf{V}^{T} \tilde{\mathbf{E}}_{p} \mathbf{V}\right)\left(\mathbf{V}^{T} \tilde{\mathbf{E}}_{0} \mathbf{V}\right)^{-1} \mathbf{V}^{T} \mathbf{b}\right] \tilde{\lambda}_{1}^{m-\left(k_{2}+\cdots+k_{p}\right)} \tilde{\lambda}_{2}^{k_{2}} \ldots \tilde{\lambda}_{p}^{k_{p}}\right\}
$$

First use Lemma 3 and then Lemma 1, it can be clearly seen that each moment of the reduce system transfer function B.3 matches the corresponding moments of the original system B.1 Since Lemma 1 and Lemma 2 hold for first q moments corresponding to $m=0,1, \ldots, m_{q}$, hence those moments are guaranteed to be matched.

For a detailed account of the proof, [54] can be referred to. 


\section{Appendix $\mathrm{C}$}

\section{Mathematical Proof of Theorem 3}

As from [90], let's expand the matrix pencil $\left(\mathbf{G}+s_{0} \mathbf{C}\right)$ at an expansion point such that the matrix pencil is non singular. So, the input to the state transfer function of the $i$-th cluster can be define as

$$
\mathbf{H}_{i}(s)=\left(\mathbf{G}_{i}+s \mathbf{C}_{i}\right)^{-1} \mathbf{B}_{i}
$$

With the Taylor series expansion of $\left(\mathbf{G}_{i}+s \mathbf{C}_{i}\right)^{-1}$ term in (C.1), $\mathbf{H}_{i}(s)$ can be rewrite as

$$
\mathbf{H}_{i}(s)=\sum_{j=0}^{\infty}(-1) \mathbf{M}_{i}\left(s_{0}\right)\left(s-s_{0}\right)^{j}
$$

The $j$-th moment of the function at $s_{0}$ is write as:

$$
\mathbf{M}_{i}\left(s_{0}\right)=\mathbf{A}_{i}^{j} \mathbf{R}_{i}
$$

where

$$
\mathbf{A}_{i}=\left(\mathbf{G}_{i}+s_{0} \mathbf{C}_{i}\right)^{-1} \mathbf{C}_{i} \quad \mathbf{R}_{i}=\left(\mathbf{G}+s_{0} \mathbf{C}\right)^{-1} \mathbf{B}_{i}
$$


note, that the $\mathbf{A}_{i}$ is going to same for all the clusters because $\mathbf{G}_{i}$ and $\mathbf{C}_{i}$ are same for all the clusters.

same way the transfer function of the reduced $i$-th cluster (5.13) can be expand at $s_{0}$ and define as:

$$
\begin{gathered}
\hat{\mathbf{H}}_{i}(s)=\sum_{j=0}^{\infty}(-1) \hat{\mathbf{M}}_{i}\left(s_{0}\right)\left(s-s_{0}\right)^{j} \\
\hat{\mathbf{M}}_{i}\left(s_{0}\right)=\mathbf{V}_{i} \hat{\mathbf{A}}^{j} \hat{\mathbf{R}}_{i}
\end{gathered}
$$

where

$$
\hat{\mathbf{A}}_{i}=\left(\hat{\mathbf{G}}_{i}+s_{0} \hat{\mathbf{C}}_{i}\right)^{-1} \hat{\mathbf{C}}_{i} \quad \hat{\mathbf{R}}_{i}=\left(\hat{\mathbf{G}}_{i}+s_{0} \hat{\mathbf{C}}_{i}\right)^{-1} \hat{\mathbf{B}}_{i}
$$

Next, we have to prove that the first $\chi_{i}=\left\lfloor q_{i} / m_{i}\right\rfloor$ block moments of (C.2) match with the (C.5).

To prove Theorem 3, first, we have to define some proposition and Lemma.

Proposition 3. The matrix $\mathbf{V}_{i}$ can be define such that the columns span by the $\mathbf{V}_{i}$ is Krylov subspace:

$$
\operatorname{colsp}\left(\mathbf{V}_{i}\right)=\operatorname{span}\left\{\mathbf{R}_{i}, \mathbf{A}_{i} \mathbf{R}_{i}, \ldots, \mathbf{A}_{i}^{\chi_{i-1}} \mathbf{R}_{i}\right\}
$$

where $\chi_{i}=\left\lfloor q_{i} / m_{i}\right\rfloor$, Also, an upper Hessenberg $H_{i, j}$ such that hold the following relation:

$$
\mathbf{A}_{i}^{j} \mathbf{R}_{i}=\mathbf{V}_{i} \mathbf{H}_{i, j}, 0 \leq j \leq \chi_{i}
$$

Proof: The equation (C.8) state that, for a $j$-th moment, there is a projection of that moment in the Krylov subspace. So, the $N \times m_{i}$ matrix $\mathbf{H}_{i, j}$ is the projection of the block moment $\mathbf{M}_{i, j}$ into Krylov subspace. So,

$$
\mathbf{M}_{i, j}=\mathbf{V}_{i} \mathbf{H}_{i, j}
$$


Substituting the value of (C.6) in (C.9) proves the relation (C.9).

Lemma 4. For the projection matrix $\mathbf{V}_{i}$ used in Proposition 3, a matrix $\mathbf{F}_{i}$ exist

$$
\mathbf{F}_{i}=\mathbf{V}_{i}\left(\mathbf{V}_{i}^{* \top} \mathbf{G}_{i} \mathbf{V}_{i}+s_{0} \mathbf{V}_{i}^{* \top} \mathbf{C}_{i} \mathbf{V}_{i}\right)^{-1} \mathbf{V}_{i}^{* \top} \mathbf{C}_{i}
$$

that satisfy the following relation:

$$
\mathbf{F}_{i}^{j} \mathbf{R}_{i}=\mathbf{A}_{i}^{j} \mathbf{R}_{i} \quad 0 \leq j<\chi_{i}
$$

Proof: Lemma 4 can be prove by mathematical induction. It is trivial for proving for $j=0$. So, let do for the $j-1$ where $0<0<\chi_{i}$

$$
\mathbf{F}_{i}^{j-1} \mathbf{R}_{i}=\mathbf{A}_{i}^{j-1} \mathbf{R}_{i}
$$

multiplying (C.13) with the $\mathbf{A}_{i}$

$$
\mathbf{A}_{i} \mathbf{F}_{i}^{j-1} \mathbf{R}_{i}=\mathbf{A}_{i}^{j} \mathbf{R}_{i}
$$

substituting the (C.4) on the left side of (C.14)

$$
\left(\mathbf{G}_{i}+s_{0} \mathbf{C}_{i}\right)^{-1} \mathbf{C}_{i} \mathbf{F}_{i}^{j-1} \mathbf{R}_{i}=\mathbf{A}_{i}^{j} \mathbf{R}_{i}
$$

used the result (C.9) from Proposition 3

$$
\left(\mathbf{G}_{i}+s_{0} \mathbf{C}_{i}\right)^{-1} \mathbf{C}_{i} \mathbf{F}_{i}^{j-1} \mathbf{R}_{i}=\mathbf{V}_{i} \mathbf{H}_{i, j}
$$

multiple the $(N \times N)$ matrix $\mathbf{V}_{i}\left(\mathbf{V}_{i}^{* \top} \mathbf{G}_{i} \mathbf{V}_{i}+s_{0} \mathbf{V}_{i}^{* \top} \mathbf{C}_{i} \mathbf{V}_{i}\right)^{-1} \mathbf{V}_{i}^{* \top}\left(\mathbf{G}_{i}+s_{0} \mathbf{C}_{i}\right)$ on the both side of (C.16) 


$$
\begin{array}{r}
\mathbf{V}_{i}\left(\mathbf{V}_{i}^{* \top} \mathbf{G}_{i} \mathbf{V}_{i}+s_{0} \mathbf{V}_{i}^{* \top} \mathbf{C}_{i} \mathbf{V}_{i}\right)^{-1} \mathbf{V}_{i}^{* \top}\left(\mathbf{G}_{i}+s_{0} \mathbf{C}_{i}\right) \times\left(\mathbf{G}_{i}+s_{0} \mathbf{C}_{i}\right)^{-1} \mathbf{C}_{i} \mathbf{F}_{i}^{j-1} \mathbf{R}_{i} \\
=\mathbf{V}_{i}\left(\mathbf{V}_{i}^{* \top} \mathbf{G}_{i} \mathbf{V}_{i}+s_{0} \mathbf{V}_{i}^{* \top} \mathbf{C}_{i} \mathbf{V}_{i}\right)^{-1} \mathbf{V}_{i}^{* \top}\left(\mathbf{G}_{i}+s_{0} \mathbf{C}_{i}\right) \mathbf{V}_{i} \mathbf{H}_{i, j}
\end{array}
$$

where $^{*}$ is the conjugate transpose simplifying the (C.17)

$$
\mathbf{V}_{i}\left(\mathbf{V}_{i}^{* \top} \mathbf{G}_{i} \mathbf{V}_{i}+s_{0} \mathbf{V}_{i}^{* \top} \mathbf{C}_{i} \mathbf{V}_{i}\right)^{-1} \mathbf{V}_{i}^{* \top} \mathbf{C}_{i} \mathbf{F}_{i}^{j-1} \mathbf{R}_{i}=\mathbf{V}_{i} \mathbf{H}_{i, j}
$$

form the (C.9), (C.11) and (C.18) the statement (C.12) in Lemma 4 is proved.

Now, let come back to prove the Theorem 3 .

So, our main purpose here is to prove that first $\chi_{i}$ block moments of the original $i$-th cluster match the same block moments of the reduced $i$-th cluster.

$$
\mathbf{M}_{i, j}\left(s_{0}\right)=\hat{\mathbf{M}}_{i, j}\left(s_{0}\right) \quad 0 \leq j \leq \chi_{i}
$$

As previous, we use Mathematical Induction method to prove the theorem. Start from $j=0$, use Proposition 3

$$
\mathbf{R}_{i}=\mathbf{V}_{i} \mathbf{H}_{i, 0}
$$

multiply $\mathbf{V}_{i}\left(\mathbf{V}_{i}^{* \top} \mathbf{G}_{i} \mathbf{V}_{i}+s_{0} \mathbf{V}_{i}^{* \top} \mathbf{C}_{i} \mathbf{V}_{i}\right)^{-1} \mathbf{V}_{i}^{* \top}\left(\mathbf{G}_{i}+s_{0} \mathbf{C}_{i}\right)$ on the both side of (C.20) and use Lemma 4

$$
\begin{aligned}
\mathbf{V}_{i}\left(\mathbf{V}_{i}^{* \top} \mathbf{G}_{i} \mathbf{V}_{i}+s_{0} \mathbf{V}_{i}^{* \top} \mathbf{C}_{i} \mathbf{V}_{i}\right)^{-1} \mathbf{V}_{i}^{* \top}\left(\mathbf{G}_{i}+s_{0} \mathbf{C}_{i}\right) \times \mathbf{R}_{i} \\
\quad=\mathbf{V}_{i}\left(\mathbf{V}_{i}^{* \top} \mathbf{G}_{i} \mathbf{V}_{i}+s_{0} \mathbf{V}_{i}^{* \top} \mathbf{C}_{i} \mathbf{V}_{i}\right)^{-1} \mathbf{V}_{i}^{* \top}\left(\mathbf{G}_{i}+s_{0} \mathbf{C}_{i}\right) \mathbf{V}_{i} \mathbf{H}_{i, 0}
\end{aligned}
$$

form (C.4) substitute $R_{i}$

$$
\mathbf{V}_{i}\left(\mathbf{V}_{i}^{* \top} \mathbf{G}_{i} \mathbf{V}_{i}+s_{0} \mathbf{V}_{i}^{* \top} \mathbf{C}_{i} \mathbf{V}_{i}\right)^{-1} \mathbf{V}_{i}^{* \top}\left(\mathbf{G}_{i}+s_{0} \mathbf{C}_{i}\right) \times\left(\mathbf{G}_{i}+s_{0} \mathbf{C}_{i}\right)^{-1} \mathbf{B}_{i}
$$




$$
=\mathbf{V}_{i}\left(\mathbf{V}_{i}^{* \top} \mathbf{G}_{i} \mathbf{V}_{i}+s_{0} \mathbf{V}_{i}^{* \top} \mathbf{C}_{i} \mathbf{V}_{i}\right)^{-1} \mathbf{V}_{i}^{* \top}\left(\mathbf{G}_{i}+s_{0} \mathbf{C}_{i}\right) \mathbf{V}_{i} \mathbf{H}_{i, 0}
$$

simplifying

$$
\mathbf{V}_{i}\left(\mathbf{V}_{i}^{* \top} \mathbf{G}_{i} \mathbf{V}_{i}+s_{0} \mathbf{V}_{i}^{* \top} \mathbf{C}_{i} \mathbf{V}_{i}\right)^{-1} \mathbf{V}_{i}^{* \top} \mathbf{B}_{i}=\mathbf{V}_{i} \mathbf{H}_{i, 0}
$$

again substituting (C.20) back into (C.23)

$$
\mathbf{V}_{i}\left(\mathbf{V}_{i}^{* \top} \mathbf{G}_{i} \mathbf{V}_{i}+s_{0} \mathbf{V}_{i}^{* \top} \mathbf{C}_{i} \mathbf{V}_{i}\right)^{-1} \mathbf{V}_{i}^{* \top} \mathbf{B}_{i}=\mathbf{R}_{i}
$$

use the (5.13), (C.7) and (C.24)

$$
\mathbf{V}_{i} \hat{\mathbf{R}}_{i}=\mathbf{R}_{i}
$$

Finally, the moments of the original and reduced system can be define as in (C.3) and (C.6). Rewrite the (C.25) as $\mathbf{M}_{i, 0}=\hat{\mathbf{M}}_{i, 0}$ which exhibit the Theorem 3 hold true for $j=0$.

Lastly, let prove (C.20) for any $\mathrm{j}$ where $0<j<\chi_{i}$. We know from the (C.6) and (C.7) that

$$
\hat{\mathbf{M}}_{i, j}=\mathbf{V}_{i} \times\left(\left(\hat{\mathbf{G}}_{i}+s_{0} \hat{\mathbf{C}}_{i}\right)^{-1} \hat{\mathbf{C}}_{i}\right)^{j} \times\left(\hat{\mathbf{G}}_{i}+s_{0} \hat{\mathbf{C}}_{i}\right)^{-1} \hat{\mathbf{B}}_{i}
$$

From the (5.13) used the value of each system matrices to substitute in to (C.26)

$$
\begin{aligned}
\hat{\mathbf{M}_{i, j}=} \mathbf{V}_{i}\left(\left(\mathbf{V}_{i}^{* \top} \mathbf{G}_{i} \mathbf{V}_{i}+\right.\right. & \left.\left.s_{0} \mathbf{V}_{i}^{* \top} \mathbf{C}_{i} \mathbf{V}_{i}\right)^{-1} \mathbf{V}_{i}^{* \top} \mathbf{C}_{i} \mathbf{V}_{i}\right)^{j} \\
& \left.\times\left(\mathbf{V}_{i}^{* \top} \mathbf{G}_{i} \mathbf{V}_{i}+s_{0} \mathbf{V}_{i}^{* \top} \mathbf{C}_{i} \mathbf{V}_{i}\right)^{-1} \mathbf{V}_{i}^{* \top} \mathbf{C}_{i} \mathbf{V}_{i}\right)^{-1} \mathbf{V}_{i}^{* \top} \mathbf{B}_{i}
\end{aligned}
$$




$$
\begin{aligned}
& \hat{\mathbf{M}}_{i, j}=\left(\mathbf{V}_{i}\left(\mathbf{V}_{i}^{* \top} \mathbf{G}_{i} V_{i}+s_{0} \mathbf{V}_{i}^{* \top} \mathbf{C}_{i} \mathbf{V}_{i}\right)^{-1} \mathbf{V}_{i}^{* \top} \mathbf{C}_{i}\right)^{j} \\
& \left.\times \mathbf{V}_{i}\left(\mathbf{V}_{i}^{* \top} \mathbf{G}_{i} \mathbf{V}_{i}+s_{0} \mathbf{V}_{i}^{* \top} \mathbf{C}_{i} \mathbf{V}_{i}\right)^{-1} \mathbf{V}_{i}^{* \top} \mathbf{C}_{i} \mathbf{V}_{i}\right)^{-1} \mathbf{V}_{i}^{* \top} \mathbf{B}_{i}
\end{aligned}
$$

with help of (C.11), (C.24) and (C.28), we get

$$
\hat{\mathbf{M}}_{i, j}=\mathbf{F}_{i}^{j} \mathbf{R}_{i}
$$

in the end use the result form Lemma 4, injunction with (C.3) and substituting in (C.29) proves the (C.19). 


\section{Appendix D}

\section{Mathematical Proof of Theorem 4}

A Final response of the original system can be compute by superimposing the individual output of each subsystem [90].

$$
\mathbf{Y}(s)=\sum_{i=1}^{K} \mathbf{Y}_{i}(s)
$$

Hence, the transfer function of original system can be define as:

$$
\mathbf{H}(s) \mathbf{U}(s)=\mathbf{H}_{1}(s) \mathbf{U}_{1}(s)+\mathbf{H}_{2}(s) \mathbf{U}_{2}(s)+\cdots+\mathbf{H}_{K}(s) \mathbf{U}_{K}(s)
$$

writing (D.2) in matrix form

$$
\mathbf{H}(s) \mathbf{U}(s)=\left[\mathbf{H}_{1}(s), \mathbf{H}_{2}(s), \ldots, \mathbf{H}_{K}(s)\right]\left[\begin{array}{c}
\mathbf{U}_{1}(s) \\
\mathbf{U}_{2}(s) \\
\vdots \\
\mathbf{U}_{K}(s)
\end{array}\right]
$$


The Laplace transformation of the (5.10) $i$-th cluster, which is input to output transfer function

$$
\mathbf{H}_{i}(s)=\mathbf{L}\left(\mathbf{G}_{i}+s \mathbf{C}_{i}\right)^{-1} \mathbf{B}_{i}
$$

Also, consider the input to state transfer function of the $i$-th cluster (5.22) as

$$
\underline{\mathbf{H}}_{i}(s)=\left(\mathbf{G}_{i}+s \mathbf{C}_{i}\right)^{-1} \mathbf{B}_{i}
$$

Combining the (D.4) and (D.5)

$$
\mathbf{H}_{i}(s)=\underline{\mathbf{L}}_{i}(s)
$$

Form (D.3) and (D.6)

$$
\begin{aligned}
\mathbf{H}(s) & =\left[\mathbf{H}_{1}(s), \mathbf{H}_{2}(s), \ldots, \mathbf{H}_{K}(s)\right] \\
& =\mathbf{L}\left[\underline{\mathbf{H}}_{1}(s), \underline{\mathbf{H}}_{2}(s), \ldots, \underline{\mathbf{H}}_{K}(s)\right]
\end{aligned}
$$

Now, expanding each term in the (D.7) by Taylor series at an expansion point $s_{0}$ and considering the (5.5)

$$
\begin{aligned}
\mathbf{H}(s) & =\mathbf{L} \sum_{j}(-1)^{j} \mathbf{M}_{j}\left(s_{0}\right)\left(s-s_{0}\right)^{j} \\
& =\sum_{j}(-1)^{j} \mathbf{L}\left[\mathbf{M}_{1, j}\left(s_{0}\right), \mathbf{M}_{2, j}\left(s_{0}\right), \ldots, \mathbf{M}_{K, j}\left(s_{0}\right)\right]\left(s-s_{0}\right)^{j}
\end{aligned}
$$

comparing the first and second part of the (D.8), the moments of the original system can be define in terms of

$$
\mathbf{M}_{j}\left(s_{0}\right)=\mathbf{L}\left[\mathbf{M}_{1, j}\left(s_{0}\right), \mathbf{M}_{2, j}\left(s_{0}\right), \ldots, \mathbf{M}_{K, j}\left(s_{0}\right)\right]
$$


By using the relation in (C.19), it is

$$
\mathbf{M}_{j}\left(s_{0}\right)=\mathbf{L}\left[\hat{\mathbf{M}}_{1, j}\left(s_{0}\right), \hat{\mathbf{M}}_{2, j}\left(s_{0}\right), \ldots, \hat{\mathbf{M}}_{K, j}\left(s_{0}\right)\right]
$$

The input to output transfer function of the reduced system in (5.19) can be write as

$$
\hat{\mathbf{H}}_{i}(s)=\hat{\mathbf{L}}_{i}\left(\hat{\mathbf{G}}_{i}+s \hat{\mathbf{C}}_{i}\right)^{-1} \hat{\mathbf{B}}_{i}
$$

as we know the value of $\hat{\mathbf{L}}_{i}$ from the (5.19)

$$
\hat{\mathbf{H}}_{i}(s)=\mathbf{L V}_{i}\left(\hat{\mathbf{G}}_{i}+s \hat{\mathbf{C}}_{i}\right)^{-1} \hat{\mathbf{B}}_{i}
$$

For the sake of simplicity the term $\hat{\mathbf{H}}_{i}(s)$ in (D.12) is shown as $\underline{\hat{\mathbf{H}}}_{i}(s)$ and compare with (5.23)

$$
\hat{\mathbf{H}}_{i}(s)=L \underline{\hat{\mathbf{H}}}_{i}(s)
$$

Also, for the response of the reduced system can be same write as (D.1)

$$
\hat{\mathbf{Y}}(s)=\sum_{i=1}^{K} \hat{\mathbf{Y}}_{i}(s)
$$

follow the same (D.2)-(D.12) step for the (D.14), we get

$$
\hat{\mathbf{H}}(s)=\mathbf{L}\left[\hat{\mathbf{H}}_{1}(s), \ldots, \hat{\mathbf{H}}_{K}(s)\right]
$$


Now, expanding each term in the (D.15) by Taylor series at an expansion point $s_{0}$ and considering the (5.5)

$$
\begin{aligned}
\hat{\mathbf{H}}(s) & =\sum_{j}(-1)^{j} \hat{\mathbf{M}}_{j}\left(s_{0}\right)\left(s-s_{0}\right)^{j} \\
& =\sum_{j}(-1)^{j} L\left[\hat{\mathbf{M}}_{1, j}\left(s_{0}\right), \hat{\mathbf{M}}_{2, j}\left(s_{0}\right), \ldots, \hat{\mathbf{M}}_{K, j}\left(s_{0}\right)\right]\left(s-s_{0}\right)^{j}
\end{aligned}
$$

as discussed before, comparing both part of (D.16)

$$
\hat{\mathbf{M}}_{j}\left(s_{0}\right)=\mathbf{L}\left[\hat{\mathbf{M}}_{1, j}\left(s_{0}\right), \hat{\mathbf{M}}_{2, j}\left(s_{0}\right), \ldots, \hat{\mathbf{M}}_{K, j}\left(s_{0}\right)\right]
$$

Finally, form the (D.10) and (D.17) we can conclude that the each entry in the moments matrix $\mathbf{M}_{j}\left(s_{0}\right)$ match the corresponding entry in the $\hat{\mathbf{M}}_{j}\left(s_{0}\right)$. This prove the Theorem 6 .

$$
\mathbf{M}_{j}\left(s_{0}\right)=\hat{\mathbf{M}}_{j}\left(s_{0}\right)
$$

